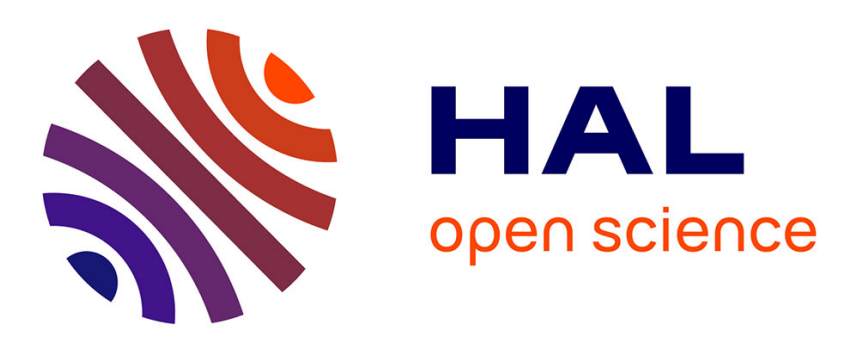

\title{
S-c-S junctions as nonlinear elements of microwave receiving devices
}

\author{
A.N. Vystavkin, V.N. Gubankov, L.S. Kuzmin, K.K. Likharev, V.V. Migulin, \\ V.K. Semenov
}

\section{- To cite this version:}

A.N. Vystavkin, V.N. Gubankov, L.S. Kuzmin, K.K. Likharev, V.V. Migulin, et al.. S-c-S junctions as nonlinear elements of microwave receiving devices. Revue de Physique Appliquée, 1974, 9 (1), pp.79-109. 10.1051/rphysap:019740090107900 . jpa-00243781

\section{HAL Id: jpa-00243781 https://hal.science/jpa-00243781}

Submitted on 1 Jan 1974

HAL is a multi-disciplinary open access archive for the deposit and dissemination of scientific research documents, whether they are published or not. The documents may come from teaching and research institutions in France or abroad, or from public or private research centers.
L'archive ouverte pluridisciplinaire HAL, est destinée au dépôt et à la diffusion de documents scientifiques de niveau recherche, publiés ou non, émanant des établissements d'enseignement et de recherche français ou étrangers, des laboratoires publics ou privés. 


\title{
S-c-S JUNCTIONS AS NONLINEAR ELEMENTS OF MICROWAVE RECEIVING DEVICES
}

\author{
A. N. VYSTAVKIN, V. N. GUBANKOV, L. S. KUZMIN \\ Institute of Radioengineering and Electronics \\ of the Academy of Sciences of the USSR, Marx avenue 18, Moscow K-9, USSR
}

K. K. LIKHAREV, V. V. MIGULIN and V. K. SEMENOV

Moscow State University, Physics department, Moscow B-234, USSR

\begin{abstract}
Résumé. - On analyse les phénomènes qui peuvent se produire dans une jonction S-c-S (supraconducteur-constriction-supraconducteur), couplée à un circuit hyperfréquence. On étudie et on discute les propriétés des jonctions, comparées à celles des éléments non linéaires usuels en hyperfréquence. On obtient les caractéristiques des récepteurs (amplificateurs, détecteurs, ou changeurs de fréquence) utilisant ces phénomènes. Les caractéristiques limites de ces dispositifs dans différents régimes de fonctionnement sont analysées et comparées avec les caractéristiques obtenues expérimentalement.

On montre que les propriétés de bruit de ces dispositifs utilisant des jonctions S-c-S quasi idéales, peuvent être obtenues pour des fréquences inférieures à la fréquence caractéristique de la jonction. Cette fréquence est de l'ordre de 500 à $1000 \mathrm{GHz}$ pour les contacts à pointes ou les microponts en couche mince.

Abstract. - The analysis of possible phenomena in the superconductor-constriction-superconductor (S-c-S) junctions inserted into microwave circuits is carried out. The results of experiments in which properties of the junctions making them different in comparison with usual nonlinear microwave elements were studied are discussed. The characteristics of receiving devices such as amplifiers, detectors and converters operating on the basis of these phenomena are obtained. The limit characteristics of these devices in different regimes of operation are analysed and compared with experimentally achieved characteristics.

It is shown that the noise properties of the receiving devices utilizing the S-c-S junctions close to ideal can be obtained for the frequencies lower than characteristic frequency of the junction. This frequency for known realizations of the S-c-S junctions (point contacts and thin film bridges of small dimensions) can reach of order of $500-1000 \mathrm{GHz}$ or in other words of the middle of the submillimeter waveband range.
\end{abstract}

1. The introduction. - 1.1 THE S-c-S JUNCTION AS AN « IDEAL » SUPERCONDUCTING ELEMENT OF THE RECEIVING DEVICES. - The most of receiving devices utilizing Josephson effect realized for this moment are made on the basis of the S-c-S junctions. We define the S-c-S (superconductor-constriction-superconductor) junction [1] as a structure in which weak link of two superconducting electrodes is created in the form of short circuit section of the same superconductor as electrodes with effective dimension $\Lambda$ less than coherence length $\xi$. The point contacts (see the review [2]) and thin film bridges of small dimensions [3]-[8] are well-known realizations of the S-c-S junctions.

It is not difficult to understand why the S-c-S junctions are wide-spread. The main requirements to nonlinear elements of superhighsensitive microwave receiver are the following. (i) The nonlinearity of high magnitude, for instance, maximum modulation coefficient for parametric elements (see, for instance [9]-[11]) or maximum curvature of $i-v$ characteristic for detectors [12].

(ii) Low level of intrinsic noise.

(iii) High cutoff frequency above which properties (i) and (ii) become get worse by some reason.

The Josephson elements satisfy well the requirement (i). As it will be shown later the magnitude of nonlinearity of these elements is limited by fluctuations only. The intrinsic noise (requirement (ii)) is low for all known superconducting elements not taking into account flicker-effect at low frequencies in the point contacts [13] and granular superconductors [14]. Therefore the main parameter reasonable for comparison of different elements is the cutoff frequency. 
In the principle the Josephson elements have the highest cutoff frequency among all superconducting elements. In fact the nonlinear processes in these elements, as it is easy to see, for instance, from [15] or [16], are caused by the interference of wave functions of two superconducting electrodes in the region of the junction and are not connected with relaxation processes of the parameter of the order $\Delta$. The relaxation of $\Delta$ can be very slow (according to the modern representations [17] it is characterized by the time constant of order of $10^{-8} \mathrm{~s}$ ) and this should limit cutoff frequency at the level of about $10^{8} \mathrm{~Hz}$ for the elements which have nonlinearity mechanim caused by this relaxation process. But Josephson elements have only one principal time constant $\tau_{0}$ which is connected with the current redistribution process in the superconductor between normal and superconducting components

$$
\tau_{0}=f_{0}^{-1}, f_{0}=\frac{2 \mathrm{e}}{h} V_{0}, \quad V_{0}=I_{0} R .
$$

In (1.1) $I_{0}$ is the critical current of the Josephson junction, $R$ is its resistance in normal state.

According to [16] and [18] at any rate at $T \approx T_{\mathrm{c}}$ this time is the same for S-I-S (tunnel) and S-c-S structures because

$V_{0}=\frac{\pi}{4} \frac{\Delta^{2}}{\mathrm{e} k T_{\mathrm{c}}} \simeq 640\left[\mu \mathrm{V} \cdot \mathrm{K}^{-1}\right] \times\left(T_{\mathrm{c}}-T\right)[\mathrm{K}]$.

The theory of the S-c-S structures at $T \rightarrow 0$ is not exist yet but according to experimental data [2] $V_{0}$ for these structures is close to $V_{0}$ for the S-I-S structures

$$
V_{0}=\frac{\pi}{2} \frac{\Delta}{\mathrm{e}} .
$$

Numerical values of $f_{0}$ obtained from (1.1)-(1.3) are in the range of $10^{11}-10^{12} \mathrm{~Hz}$.

However not all Josephson elements have such high cutoff frequency. For instance the cutoff frequency of the S-I-S junction $\left({ }^{1}\right)$ is the plasma frequency [1] which is determined by parasitic capacitance $C$ of the junction

$$
\tau_{\mathrm{p}}=2 \pi\left(\hbar C / 2 \mathrm{e} I_{0}\right)^{1 / 2} \gg \tau_{0} .
$$

The values of $\Delta$ in S-S'-S structures, for instance in bridge type structures [19]-[22], are making damped artificially that makes values $V_{0}$ lower, down to $\left(10^{-6}-10^{-5}\right) \mathrm{V}$, i. e. makes values $f_{0}$ lower, down to

(1) We mean here the junctions of small cross-section dimensions ( $\widetilde{<} \lambda J-$ wavelength of Josephson oscillations) which are operating as lumped elements. The characteristics of the tunnel junction receiving elements operating in traveling-wave regime should be high. But realization of effective microwave power interchange between such elements and external circuits is remaining difficult problem. $\left(10^{9}-10^{10}\right) \mathrm{Hz}$. The values of $V_{0}$ for the S-N-S structures naturally are also low [1], [23]-[28]. The values of $V_{0}$ for the bridges superimposed on the nickel [29] also get two-three times lower.

By exactly this reason the S-c-S junctions are the most promising superconducting nonlinear elements for the microwave range (we mean under microwaves here the short wave length portion of the centimeter waveband range and also the millimeter and submillimeter waveband range $\left({ }^{2}\right)$ ). This work is devoted to the analysis of these junctions.

1.2 The MOdel of the S-c-S JUNCtion. - We will use in the theoretical analysis of processes in the S-c-S junctions well-known " resistive " model proposed initially for description of the S-N-S structures [30] and the tunnel junctions with small capacitance [31], [32]. The validity of this model for the S-c-S junctions at $T \approx T_{\mathrm{c}}$ then was proved by Aslamazov and Larkin [16]. According to this model the current $I$ through the junction is

$$
I=I_{\mathrm{s}}+I_{\mathrm{N}}=I_{0} \sin \varphi+V / R,
$$

where $\varphi$ is the wave function phase difference of superconducting electrodes forming the junction

$$
\mathrm{d} \varphi / \mathrm{d} t=(2 \mathrm{e} / \hbar) V(t),
$$

$V$ the voltage across the junction.

The resistive model explaines well, as it was demonstrated many times, almost all known properties of the S-c-S junctions of dimensions $\Lambda \approx \xi$. Moreover this model is "ideal " in some sense because practically any deflection from it deteriorates microwave properties of the junctions.

The necessity of taking into account the fluctuations is of principle during the investigation of many properties of the junctions. It is possible to make this using the Langevin method [1] adding the fluctuation term $I_{\mathrm{f}}(t)$ to the current $I$ through junction, $\left\langle I_{\mathrm{f}}\right\rangle=0\left({ }^{3}\right)$. During the analysis we will neglect the noise of type $1 / f$ which is unimportant in any case at frequencies $\approx 10^{5} \mathrm{~Hz}$ [13]. Then at not too large voltages across the junction and not too high frequencies such as

$$
\mathrm{e} V, \hbar \omega \approx k T
$$

the intrinsic noise of junction can be considered as the white (hf) noise

$$
<I_{\mathrm{f}} \cdot I_{\mathrm{f}_{\tau}}>=\frac{2 k T}{R} \delta(\tau) .
$$

(2) By the same reason, as we have shown recently, using S-c-S junctions it is possible to create the most high speed elements of computers with switch time of order of $1 \mathrm{ps.}$

(3) We will define averaging procedure on ensemble using the brackets $\langle\cdots\rangle$ and averaging procedure on time using upper line. The subscript $\tau$ means that given value is taken at time $t+\tau$. 
Since $\mathrm{e} V, \hbar \omega \simeq \mathrm{e} V_{0}$ for all real regimes of using the junctions actual condition of applicability of the expression (1.8) is the relation $V_{0} \approx V_{\mathrm{T}}=k T / \mathrm{e} . V_{\mathrm{T}} \simeq 360 \mu \mathrm{V}$ for $T=4.2 \mathrm{~K}$ and this condition is fulfilled for many realizations of the S-c-S junctions. However recently the values of $V_{0} \simeq 1 \mu \mathrm{V}$ were achieved as for the point contacts [2] so for the bridges of small dimensions [33]. In this case the value of noise may be greater than it gives the expression (1.8). However the temperature cannot be higher than $\mathrm{e} V / 2 k$ [34], [35] even in the most unfavourable case when the electron free path length $l \gg \Lambda$. The noise is lower in case when $l \ll \Lambda[36]$. By this reason we will restrict the consideration during the analysis by the expression (1.8) assuming that $T$ stands in general case for the effective temperature which can be somewhat higher than physical temperature of the junction at large bias voltages.

Sometimes taking into account the external fluctuations (interference) arising in the junction from external circuits is essential. Usualy spectral components at frequencies much lower than operating frequencies of the microwave devices $\left(\approx 10^{8} \mathrm{~Hz}\right)$ prevail in the external fluctuations. In this case it is possible to assume that the correlation time of $I_{\mathrm{f}}$ is large (the noise is the lf noise).

It means [37] that it is possible to calculate during the analysis any value $F$ at the absence of the external fluctuations as a function of the bias current $I$ and then to calculate total value $\langle F\rangle$ using the expression

$$
<F(\bar{I})>=\int_{-\infty}^{+\infty} F\left(\bar{I}+I_{\mathrm{f}}\right) w\left(I_{\mathrm{f}}\right) \mathrm{d} I_{\mathrm{f}} .
$$

The distribution $w\left(I_{\mathrm{f}}\right)$ is assumed to be normal

$$
w\left(I_{\mathrm{f}}\right)=\frac{1}{\sqrt{2 \pi} I_{\mathrm{f} 1}^{\prime}} \times \exp \left\{-\frac{I_{\mathrm{f}}^{2}}{2 I_{\mathrm{f} 1}^{\prime 2}}\right\},
$$

and it is possible to define the noise intensity as effective amplitude $I_{\mathrm{fl}} / 2$ [38]

$$
\int_{-I_{\mathrm{f}} / 2}^{I_{\mathrm{f} 1} / 2} w\left(I_{\mathrm{f}}\right) \mathrm{d} I_{\mathrm{f}}=\frac{1}{2}, \quad I_{\mathrm{f} 1} \simeq 1.349 I_{\mathrm{f} 1}^{\prime} .
$$

This approach to the analysis of intrinsic and external fluctuations is common and it is well suitable for explaining of experimental results.

In further analysis we will use often for the sake of simplicity the usual variable normalization procedure according which currents, voltages, impedances, frequencies and time are taking in units of $I_{0}, V_{0}, R$, $f_{0}$ (or $\omega_{0}=2 \pi f_{0}$ ) and $\tau_{0}$, respectively. Corresponding small letters are using for defining of normalized variables. The exception is made for normalized frequencies and normalized time which are defined as $\Omega$ or $\Delta\left({ }^{4}\right)$ and $t$.

(4) Do not mix up with the order parameter.
Using these dimensionless variables it is possible according to (1.5) to write down the equation for processes in the junction in the form

$$
\dot{\varphi}+\sin \varphi=\bar{i}+i_{\mathrm{f}}+i_{\mathrm{e}},
$$

where $i_{\mathrm{e}}$ is the " coupling current ", i. e. the current in the junction caused by the external microwave circuit in which the junction is inserted. Using dimensionless variables we rewrite the eq. (1.8) for the intrinsic noise in the form

$$
\left\langle i_{\mathrm{f}} . i_{\mathrm{f} \tau}>=2 \Gamma \delta(\tau),\right.
$$

where it is possible to represent the relative noise intensity $\Gamma$ either as the relative effective amplitude of the intrinsic fluctuations

$$
2 \Gamma=\frac{I_{\mathrm{fh}}}{I_{0}}, \quad I_{\mathrm{fh}}=\frac{4 \mathrm{e} k T}{\hbar}
$$

$\left(I_{\mathrm{fh}} \simeq 0.36 \mu \mathrm{A}\right.$ at $\left.T=4.2 \mathrm{~K}\right)$, or as the measure of the normal resistance of the junction

$$
\Gamma=\frac{R}{R_{\mathrm{f}}}, \quad R_{\mathrm{f}}=\frac{\hbar}{2 \mathrm{e}^{2}} \cdot \frac{\mathrm{e} V_{0}}{k T} \simeq 2.06\left(\frac{\mathrm{e} V_{0}}{k T}\right)[k \Omega] .
$$

1.3 THE SUMMARY OF THE WORK. - We carry out the theoretical analysis of electrodynamic (microwave) properties of the S-c-S junctions which can be used for constructing of sensitive microwave receiving devices using resistive model representation. We make comparison of the obtained results with the experimental data for the S-c-S junctions of different types. Besides that we consider different operating regimes of such devices and find their characteristics.

We use the following common method of analysis. Relative bandwidths of the most of the receiving devices are small for all used frequency components. This allows us to assume that the process in a device can be represented as a sum of not large amount of quasi-harmonic frequency components.

Therefore it is possible to carry out the analysis in two stages. At first we assume that the incident action on the junction is given and calculate a response for this incident action. Then we consider the external circuit and find all parameters of the process on the basis of obtained results. According to this we have divided this work into parts dependingly on intensity of incident (external) action on the junction.

The properties of the autonomous junction $\left(i_{\mathrm{e}}=0\right)$ are described in the part 2. The part 3 is devoted to the consideration of linear properties of the junction when the action of external circuit on the junction is so small that the response of the junction is proportional to the intensity of the action. Such regime is used in parametric amplifiers and converters with self-pumping and by this reason the analysis of them is carried out in the part 3 also. Quadratic effects at small external action are considered in the part 4 
and characteristics of the Josephson detectors of different types are obtained on the basis of this consideration. The part 5 is devoted to the study of some effects at strong interaction of the junction with the external circuit. In particular in the part 5 the limit power of the Josephson radiation is calculated and besides the characteristics of the parametric downconverters with external pumping are obtained. The results of the analysis of the receiving devices of different types obtained in the parts 2-5 are the subject of comparison and discussion in the part 6 .

Our references include in the main only two types of works on the Josephson effect : the experimental investigations of microwave properties of the S-c-S junctions and the theoretical studies of autonomous and microwave properties of the resistive model (without taking into account the capacitance). We cite mainly the monograph [1] regarding to other topics.

It is necessary to mention also the reviews [2], [39], [40] devoted to the same problem.

2. The properties of autonomous S-c-S junctions. The junction can be assumed as an autonomous if the variable $i_{\mathrm{e}}$ is vanishingly small. Then the process in the junction is described by the equation.

$$
\dot{\varphi}+\sin \varphi=\bar{i}+i_{\mathrm{f}},
$$

solving which one can find a voltage $v=\dot{\varphi}$ across the junction.

2.1 THE CASE WHEN THE FLUCTUATIONS ARE ABSENT. - At $i_{\mathrm{f}}=0$ the eq. (2.1) is usual « phaselocking equation " and it has well-known solution. If the bias current is less than the critical current $(|\bar{i}|<1)\left({ }^{5}\right)$ than

$$
\varphi(t) \rightarrow \arcsin \bar{i}=\text { Cte }, \quad v \rightarrow 0 .
$$

At the current exceeding the critical current $(\bar{i}>1)\left({ }^{5}\right)$ the phase changes infinitely

$$
\varphi=2 \operatorname{arctg}\left(\frac{\bar{v}}{\bar{i}+1} \operatorname{tg} \frac{\bar{v} t}{2}\right)+\frac{\pi}{2} .
$$

The expression for dc component of the voltage

$$
\bar{v}=\left(\bar{i}^{2}-1\right)^{1 / 2}
$$

gives hyperbolic portion of the $i-v$ characteristic of the junction and total expression for $v$

$$
v=\frac{\bar{v}^{2}}{\bar{i}-\cos \bar{v} t}=\bar{v}+\sum_{k=1}^{\infty} \frac{2 \bar{v}}{(\bar{i}+\bar{v})^{k}} \cos k \bar{v} t
$$

describes the process of the Josephson oscillations [1] with normalized frequency $\bar{v}$ (or with dimensional

(5) There is no distinguishable direction in the resistive model of the junction, $\varphi(-\bar{i})=-\varphi(\bar{i})$, and only this case can be considered. frequency $\omega=2 \mathrm{e} \bar{V} / \hbar)$. The Josephson radiation from the S-c-S junctions was observed first in the works [41], [42], [43]

If we observe the radiation from the junction to the microwave circuit with so large impedance $R_{\mathrm{e}}$ in comparison with the junction resistance that the autonomy is not disturbed $\left(r_{\mathrm{e}}=R_{\mathrm{e}} / R \gg 1\right)$ then according to $(2.5)$ the power of the radiation at $k$ th harmonic is

$$
p_{k}=\frac{P_{k}}{I_{0} V_{0}}=\frac{2 \bar{v}^{2}}{(\bar{i}+\bar{v})^{2 k} r_{\mathrm{e}}} .
$$

If the bias voltage across the junction is fixed (and the receiver of radiation is retuning) then the harmonic power decreases with the harmonic number $k$ according to the geometric progression

$p_{k} \sim(\bar{i}+\bar{v})^{-2 k} \simeq\left\{\begin{array}{l}(2 \bar{v})^{-2 k}, \bar{v} \sim 1 \\ \text { Cte }, \bar{v} \ll 1, k<(\bar{v})^{-1} .\end{array}\right.$

In more real case when the receiver operating frequency is fixed $\left(\Omega=\omega / \omega_{n}\right)$ and the number of measured harmonic is determined by changing bias voltage across the junction the dependence of $p_{k}$ on $k$ is more complicated

$$
\begin{aligned}
p_{k} & =\frac{2 \Omega^{2}}{k^{2}\left[\left(1+\Omega^{2} / k^{2}\right)^{1 / 2}+\Omega / k\right]^{2 k}} \\
& = \begin{cases}k^{-2}(2 \Omega / k)^{-2 k}, & \Omega \gg 1, \\
k^{-2}, & \Omega \ll 1 .\end{cases}
\end{aligned}
$$

The good agreement with the expression (2.8) was observed in our experiments [44] and also in the works [43], [45].

2.2 THE INFLUENCE OF THE FLUCTUATIONS ON THE $i$ - $v$ CHARACTERISTIC. - If the fluctuations are high-frequency (intrinsic) fluctuations then the analysis of the eq. (2.1) is carrying out using EinsteinFocker-Planck (EFP) equation

$$
\frac{\partial \sigma}{\partial t}=-\frac{\partial}{\partial \theta}[\sigma(\bar{i}+\cos \theta)]+\Gamma \frac{\partial^{2} \sigma}{\partial \theta^{2}}, \quad \theta=\varphi-\frac{\pi}{2}
$$

and it is easy to obtain average rate of phase changing for the steady-state regime

$$
\begin{aligned}
\langle\bar{v}\rangle & =\langle\overline{\dot{\varphi}}\rangle=(\Gamma / \pi) \operatorname{sh}(\pi \bar{i} / \Gamma) \cdot\left|I_{\mathrm{j} i / \Gamma}\left(\Gamma^{-1}\right)\right|^{-2} \\
& =(\Gamma / \pi) \cdot(\pi / 2) \operatorname{sh}(\pi \bar{i} / \Gamma) \\
& \int_{0}^{\pi / 2} \operatorname{ch}(2 \bar{i} x / \Gamma) I_{0}[(2 / \Gamma) \cos x] \mathrm{d} x .
\end{aligned}
$$

This result was obtained by Stratonovich [46] for the phase locking equation and then over again in the work [47]. The dependence of $\langle\bar{v}\rangle$ on $\bar{i}$ which 
is determined by eq. (2.10) is well-known dependence [46], [47], [1] and is the " hyperbolic " $i$ - $v$ characteristic smoothed down under influence of the noise.

When the noise $(\Gamma)$ increases this smoothing down process begins from the corner of the characteristic $(\bar{i}=1, \bar{v}=0)$. The rest of characteristic at $\Gamma \ll 1$ remains without change. It is possible to consider that upper limit of smoothed down portion is that value of $\langle\bar{v}\rangle$ at which dc differential resistance of the junction

$$
r_{\mathrm{d}}=\frac{\partial\langle\bar{v}\rangle}{\partial \bar{i}}
$$

is maximum. The dependences of this value of $\langle\bar{v}\rangle$ and also $\left(r_{\mathrm{d}}\right)_{\max }$ on $\Gamma$ are shown at figure 1 (solid lines) [38]. One can see that at $\Gamma \ll 1$ limit the value of $\langle\bar{v}\rangle \simeq 0.5 \Gamma^{1 / 3}$ and $\left(r_{\mathrm{d}}\right)_{\max } \sim \Gamma^{-1 / 3}$.

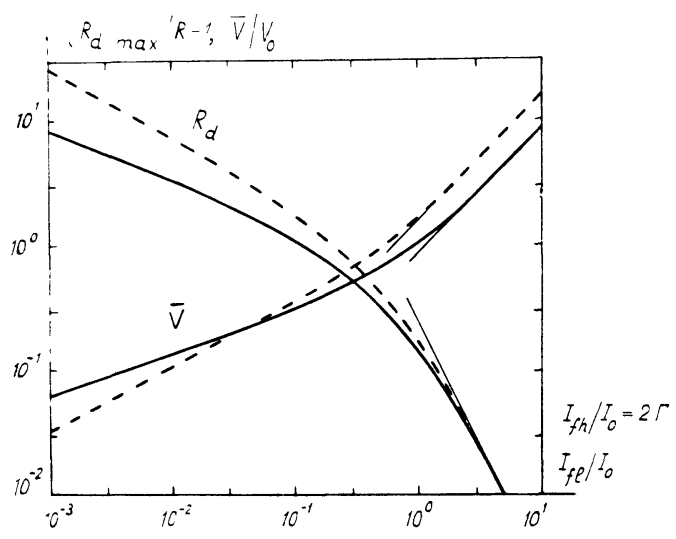

Fig. 1. - The dependences of the maximum dc differential resistance of the junction and the voltage, corresponding to this maximum, on the noise intensity. The dashed lines are for the external interferences (If fluctuations) and the solid lines are for the intrinsic thermal noise (hf fluctuations).

Calculating the $i-v$ characteristic for low-frequency (external) fluctuations using the expression (1.9), where in this case $F=\bar{v}$, we also obtain smoothed down characteristic [37]. The difference and similarity of the action of hf and if fluctuations on the junction are clearly seen from figure 1 [38], where the same dependences for if case are shown by dashed lines. The action of If and hf fluctuations is practically identical at $\bar{v} \sim 1$ but at small values of $\bar{v}$ the form of influence is different. For instance limit value of $\langle\bar{v}\rangle \sim\left(I_{\mathrm{f} 1} / I_{0}\right)^{1 / 2}$ and $\left(r_{\mathrm{d}}\right)_{\max } \sim\left(I_{\mathrm{f} 1} / I_{0}\right)^{-1 / 2}$ at small if fluctuations.

On the whole one can say that the $i-v$ characteristic is smoothed down essentially at $\Gamma \widetilde{>} 1$, i. e. at the junction resistance $R$ of order of "fluctuation " value $R_{\mathrm{f}}(1.15)$.

Comparing experimental $i-v$ characteristics of the S-c-S junctions with theoretical ones obtained on basis of the resistive model it is necessary to say that the shape of the characteristic is more sensitive to variations of the model in comparison with other properties of the junction. However the correspondence between theory and experiment is pretty good for the point contacts with values of $R$ in region $\left(10^{-1}\right.$. $\left.10^{2}\right) \mathrm{ohm}$ [2], [48] and also for the bridges with dimensions less or of order of $\xi$ [3]-[7] especially if to use experimental values of $V_{0}$. Not going into detailed analysis of reasons of deviations of real junctions from the resistive model (see [48], [49]) we should note that deviations for low resistance junctions are connected mainly with non-satisfied requirement $\Lambda \approx \xi$ and also with thermal effects, and deviations for high resistance junctions are connected with non-fulfilled assumption that $l \ll \Lambda$ and also with the influence of essentially nonlinear [1] additional tunnel current through oxide layer of contact area.

2.3 THE LINE BANDWIDTHS OF JOSEPHSON OSCILLATIONS AND THE INTRINSIC NOISE OF THE JUNCTION. As the line bandwidths of the harmonics of Josephson oscillations so the level of If fluctuations of the voltage across the junction at the action of the fluctuations can be obtained using the dimensionless spectral density of the voltage across the junction

$$
s_{v}(\Omega)=S_{V}(\omega) /\left[(2 \mathrm{e} / \hbar)^{2} / \omega_{0}\right] .
$$

It is convenient for the analysis to consider separately different values of relative intensity of fluctuations, namely to assume that the noise is " low " when the relative line bandwidth of the main (first) harmonic at given bias is small and to assume that the noise is " high » in the contrary case. It turned out [36] that it is easy to obtain $s_{v}(\Omega)$ for the «low » noise even at arbitrary shape of the spectrum of value $i_{\mathrm{f}}$.

At first consider case when $i_{\mathrm{f}}$ is small. We write down $i^{(1)}=i_{\mathrm{f}}$ and find a solution of the eq. (2.1) in the form $\varphi=\varphi^{(0)}+\varphi^{(1)}$ where $\varphi^{(0)}$ is the solution of the eq. (2.1) in the absence of the noise (2.3). Carring out the linearization of the eq. (2.1) over $\varphi^{(1)}$ we find

$$
\dot{\varphi}^{(1)}+\varphi^{(1)} \cdot \cos \varphi^{(0)}=i^{(1)} .
$$

For dc Josephson effect $(|\bar{i}|<1)$

$$
\cos \varphi^{(0)}=\left(1-\overline{i^{2}}\right)^{1 / 2}
$$

and turning to the Fourier-images $i_{\Omega}$ and $v_{\Omega}$ for the fluctuations of the current $i^{(1)}$ and the voltage $v^{(1)}=\dot{\varphi}^{(1)}$ we obtain at once

$$
v_{\Omega}=i_{\Omega}\left[1-j\left(1-\bar{i}^{2}\right)^{1 / 2} / \Omega\right]^{-1} .
$$

From (2.14) the spectral density of the voltage is

$$
s_{v}(\Omega)=s_{i}(\Omega) \cdot\left[1+\left(1-\bar{i}^{2}\right) / \Omega^{2}\right]^{-1},
$$

where $s_{i}(\Omega)$ is the dimensionless spectral density of the current fluctuations

$$
s_{i}(\Omega)=S_{I}(\omega) /\left[(2 \mathrm{e} / \hbar)^{2} R^{2} / \omega_{0}\right] .
$$


It would be $s_{v}(\Omega)=s_{i}(\Omega)$ for the normal resistance $R\left(I_{0}=0\right)$ at used normalization procedure. Therefore the expression (2.15) describes a suppression of noise in the junction during transition to superconducting state, $s_{v}(0)=0$. This suppression does not reveal itself at frequencies $\widetilde{s} \omega_{0}$.

Solving the eq. (2.13) over $v^{(1)}$ at $\bar{i}>1$ (the region of the Josephson oscillations) we obtain

$$
v^{(1)}=i^{(1)}+\ddot{\varphi}(0) \int i^{(1)} \cdot\left(\dot{\varphi}^{(0)}\right)^{-1} \mathrm{~d} t
$$

Turning to the Fourier-images again we obtain easily

$$
v_{\Omega}=\sum_{k} z_{k} \cdot i_{\Omega-k \bar{v}}, \quad \bar{v}=\overline{\dot{\varphi}^{(0)}},
$$

where $z_{k}$ are frequency conversion factors for current components caused by mixing of them with the Josephson oscillations

$$
\begin{aligned}
z_{k}= & \delta_{k, 0}+k \bar{i}(\bar{i}+\bar{v})^{-|k|} \cdot(\Omega-k \bar{v})^{-1}- \\
& -\frac{1}{2}\left\{(k-1) \cdot(\bar{i}+\bar{v})^{-|k-1|} \cdot[\Omega-(k-1) \bar{v}]^{-1}\right. \\
& \left.+(k+1) \cdot(\bar{i}+\bar{v})^{-|k+1|}[\Omega-(k+1) \bar{v}]^{-1}\right\} .
\end{aligned}
$$

We obtain from (2.18) the spectral density of the voltage

$$
s_{v}(\Omega)=\sum_{k}\left|z_{k}\right|^{2} s_{i}(\Omega-k \bar{v}) .
$$

Let us analyse now obtained result. The dependences given by (2.19), (2.20) are shown at figures $2 a$ and $2 b$ by dashed lines for case when current noise is white noise (1.8), $S_{I}(\omega)=k T / \pi R, s_{i}(\Omega)=\Gamma / \pi=$ Cte. Here $g(\Omega)$ is the ratio of the spectral density $S_{V}(\omega)$ to the spectral density which would be at normal state

$$
g(\Omega)=s_{v}(\Omega) / s_{i}=s_{v} /(\Gamma / \pi)=S(\omega) /(k T R / \pi) .
$$

We have for low frequencies $(\Omega \ll \bar{v})$

$$
s_{v}(0)=\left[\bar{i}^{2} s_{i}(0)+\frac{1}{2} s_{i}(v)\right] \bar{v}^{-2},
$$

and for frequencies near $n \bar{v}(|\Omega-n \bar{v}| \ll \bar{v})$

$$
s_{v}(\Omega)=n^{2}(\bar{i}+\bar{v})^{-2 n}\left[\bar{i}^{2} s_{i}(0)+\frac{1}{2} s_{i}(\bar{v})\right](\Omega-n \bar{v})^{-2} .
$$

The latter expression gives the lower portion shape of the Lorentz line of the Josephson oscillations $n$th harmonic. It is possible to obtain from it dimensionless bandwidth $2 \Gamma_{\mathrm{n}}$ of this line using essentially. the fact that we consider small noise and $\Gamma_{\mathrm{n}} \ll \bar{v}$. We use for this the general expression for the Lorentz line

$$
s_{v}(\Omega)=(2 \pi)^{-1} \cdot\left(p_{\mathrm{n}} r_{\mathrm{e}}\right) \Gamma_{\mathrm{n}}\left[(\Omega-n \bar{v})^{2}+\Gamma_{\mathrm{n}}^{2}\right]^{-1},
$$

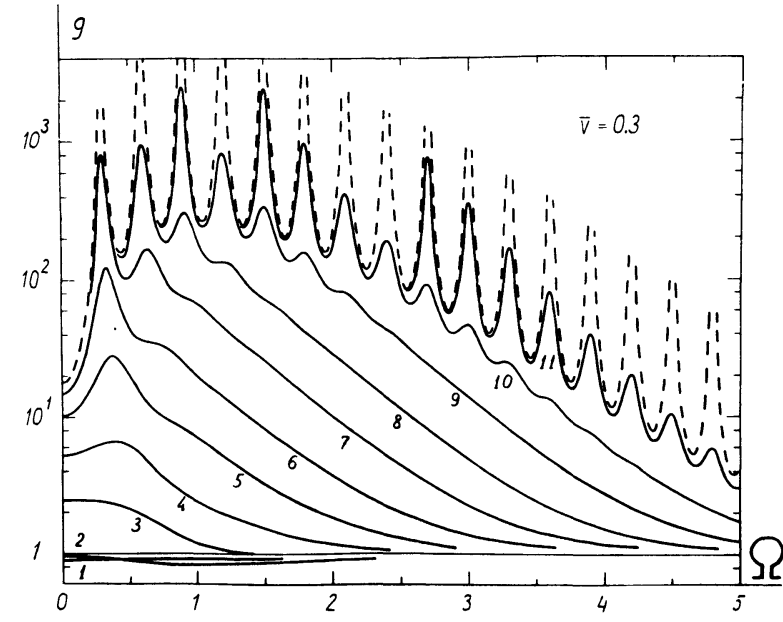

(a)

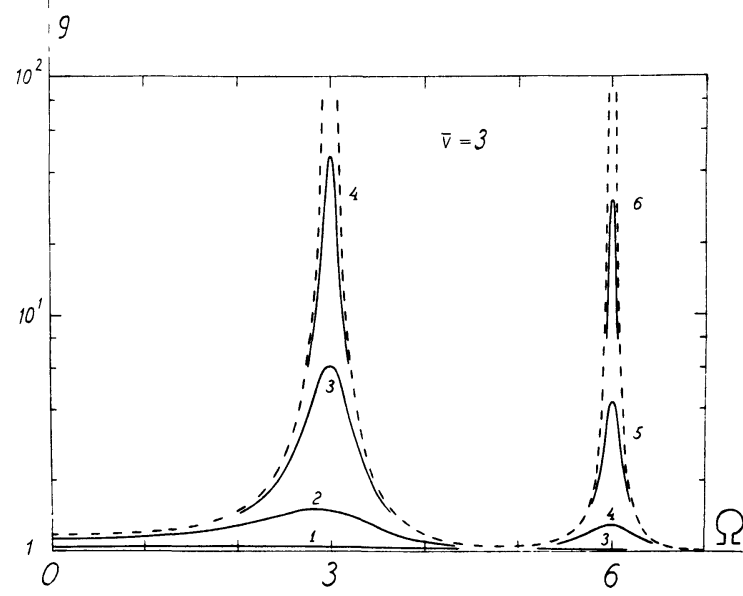

(b)

Fig. 2. - The spectral density of the fluctuations of the voltage for two values of the average voltage $\bar{v}$ across the junction : (a) $v=0.3,(b) \bar{v}=3$. The different solid lines correspond to different values of relative intensity $\Gamma$ of the current fluctuations : (1) $\Gamma=2$, (2) $6.7 \times 10^{-1}$ (and the following values of $\Gamma$ decrease in half of order each time), (3) $2 \times 10^{-1}$, (5) $2 \times 10^{-2}$, (7) $2 \times 10^{-3}$, (9) $2 \times 10^{-4}$, (11) $2 \times 10^{-5}$. The dashed line corresponds to the spectral density at very small noise $(\Gamma \rightarrow 0)$.

equate total radiation power at this harmonic

$$
p_{\mathrm{n}}=\frac{2}{r_{\mathrm{e}}} \int_{n \bar{v}-\varepsilon}^{n \bar{v}+\varepsilon} s_{v}(\Omega) \mathrm{d} \Omega, \quad \Gamma_{\mathrm{n}} \ll \varepsilon \ll \bar{v}
$$

with its value in the absence of fluctuations (2.6). Then comparing (2.23) and (2.24) for frequencies $|\Omega-n v| \gg \Gamma_{\mathrm{n}}$ we obtain the expression for half of bandwidth $\left({ }^{6}\right)$

$$
\Gamma_{\mathrm{n}}=n^{2} \Gamma_{1}=n^{2} \pi\left[\bar{i}^{2} s_{i}(0)+\frac{1}{2} s_{i}(\bar{v})\right] \bar{v}^{-2} .
$$

One can pay attention now to the fact that $\Gamma_{1}$ is simply expressed through $s_{v}(0)$

$$
\Gamma_{1}=\pi s_{v}(0) \text {. }
$$

(6) One should note that $\Gamma_{\mathrm{n}}=n \Gamma_{1}$ for the lf fluctuations. This allows to evaluate the correlation time of the noise source during the experiment. 
This equality expresses the fact that the line bandwidth of the Josephson oscillations depends as for usual oscillators [46] only on low frequency portion of voltage fluctuation spectrum from zero to frequencies of about line bandwidth of oscillations $\left(\Gamma_{1}\right)$.

Since the fluctuations do not change $\bar{v}$ in considering first order approximation and therefore dimensionless dc differential resistance is $r_{\mathrm{d}}=\mathrm{d} \bar{v} / \mathrm{d} \bar{i}=\bar{i} / v$ we can rewrite $(2.26)$ for white noise $\left(s_{i}=\Gamma / \pi\right)$ in the form

$$
\Gamma_{\mathrm{n}}=n^{2} \Gamma\left[\bar{i}^{2}+\frac{1}{2}\right] \bar{v}^{-2}=n^{2} \Gamma r_{\mathrm{d}}^{2}\left[1+\frac{1}{2} \bar{i}^{2}\right] \text {. }
$$

Thus for Josephson oscillating systems at large bias $(\bar{v} \gg 1)$ we obtain usual result [1]

$$
\Gamma_{\mathrm{n}}=n^{2} \Gamma, \quad s_{v}(0) \simeq s_{i}(0)
$$

however the voltage fluctuations and the line bandwidths of oscillations at low bias $(\bar{v} \ll 1)$ are increasing sharply

$$
\Gamma_{\mathrm{n}}=n^{2} \pi s_{v}(0)=n^{2} \frac{3}{2} \Gamma r_{\mathrm{d}}^{2}
$$

The theory for the tunnel junctions (not applicable to the S-c-S junctions) gives the same dependence for $\Gamma_{\mathrm{n}}$ on $r_{\mathrm{d}}$, namely $\Gamma_{\mathrm{n}} \sim r_{\mathrm{d}}^{2}$ [35], but the dependence of $\Gamma_{\mathrm{n}}$ on the current is quite different.

One should note now that according to (2.29) and (2.30) the condition of small value of noise at given bias is

$$
\Gamma \ll \begin{cases}\bar{v}, & \bar{v} \approx 1, \\ \bar{v}^{3}, & \bar{v} \approx 1 .\end{cases}
$$

Comparing this result with figure 1 one can see clearly that simple criterion of small value of noise exists. Namely the noise can be assumed to be smalll if the operating point on the $i-v$ characteristic is located higher than smoothed portion of it caused by fluctuations.

When the value of the noise is arbitrary it is possible to apply EFP method. We use for this well-known expression [50] for the spectral density of the process with stationary increments

$S_{\zeta}(\omega)=-\frac{1}{4 \pi} \oint_{-\infty}^{+\infty} D_{\zeta}(\tau) \exp \{-j \omega \tau\} \mathrm{d} \tau$,

where $D_{\zeta}(\tau)=\left\langle\left(\zeta_{\tau}-\zeta\right)^{2}\right\rangle$ is structural function. Hence in considering case we obtain for relative spectral density of voltage fluctuations $g(2.21)$

$$
\begin{aligned}
g=-\frac{\Omega^{2}}{2 \Gamma} \oint_{-\infty}^{+\infty} \iint\left(\theta_{1}-\theta\right)^{2} \sigma(\theta) \sigma\left(\theta \mid \theta_{1}, t\right) \times \\
\times \exp \{-j \Omega t\} \mathrm{d} t \mathrm{~d} \theta \mathrm{d} \theta_{1} .
\end{aligned}
$$

$\sigma(\theta)$ and $\sigma\left(\theta \mid \theta_{1}, t\right)$ are respectively stationary and conditional densities of phase $(\theta)$ probability which should be found from stationary and non-stationary EFP equations respectively

$$
\begin{gathered}
0=-\frac{\mathrm{d}}{\mathrm{d} \theta}[(\bar{i}+\cos \theta) \sigma(\theta)]+\Gamma \frac{\mathrm{d}^{2}}{\mathrm{~d} \theta^{2}} \sigma(\theta), \\
\frac{\partial}{\partial t} \sigma\left(\theta \mid \theta_{1}, t\right)=-\frac{\partial}{\partial \theta_{1}}\left[\left(\bar{i}+\cos \theta_{1}\right) \sigma\left(\theta \mid \theta_{1}, t\right)\right]+ \\
+\Gamma \frac{\partial^{2}}{\partial \theta_{1}} \sigma\left(\theta \mid \theta_{1}, t\right)
\end{gathered}
$$

with usual conditions

$$
\sigma(\theta)=\sigma(\theta+2 \pi), \quad \sigma\left(\theta \mid \theta_{1}, 0\right)=\delta\left(\theta-\theta_{1}\right) .
$$

We introduce the designations

$$
\begin{aligned}
B_{k}(\Omega)= & j \frac{\Omega}{2 \Gamma} \oint_{-\infty}^{+\infty} \iint\left(\theta_{1}-\theta\right) \sigma(\theta) \sigma\left(\theta \mid \theta_{1}, t\right) \times \\
& \times \exp \left\{-j\left(k \theta_{1}+\Omega t\right)\right\} \mathrm{d} t \mathrm{~d} \theta \mathrm{d} \theta_{1}, \\
\sigma_{k, 0}=j \Omega & \oint_{-\infty}^{+\infty} \iint \sigma(\theta) \sigma\left(\theta \mid \theta_{1}, t\right) \times \\
& \times \exp \left\{-j\left(k \theta_{1}+\Omega t\right)\right\} \mathrm{d} t \mathrm{~d} \theta \mathrm{d} \theta_{1} .
\end{aligned}
$$

Introduced by such manner values $\sigma_{k, 0}$ satisfy the set of equations

$$
\begin{array}{r}
\sigma_{0,0}=1,2(\bar{i}-j k \Gamma) \sigma_{k, 0}+\sigma_{k-1,0}+\sigma_{k+1,0}=0, \\
k>0, \quad \sigma_{-k, 0}=\sigma_{k, 0}^{*},
\end{array}
$$

and therefore do not depend on $\Omega(\Omega \neq 0)$. After multiplying the eq. $(2.34 b)$ by

$$
\left(\theta_{1}-\theta\right) \sigma(\theta) \exp \{-j \Omega t\}
$$

and integration it over three arguments $\left(t, \theta, \theta_{1}\right)$ we obtain

$$
g(\Omega)=1+\operatorname{Re}\left[B_{1}(\Omega)+B_{1}(-\Omega)\right] .
$$

We have now to find only an expression for $B_{k}$. We multiply for this $(2.34 b)$ by

$$
\left(\theta_{1}-\theta\right) \sigma(\theta) \exp \left\{-j\left(k \theta_{1}+\Omega t\right)\right\}
$$

and carry out integration over the same three arguments. Hence we have

$$
\begin{gathered}
B_{0}(\Omega)=-j<\bar{v}>/ 2 \Gamma \Omega, \\
2[(\bar{i}+\Omega / k)-j k \Gamma] B_{k}(\Omega)+B_{k-1}(\Omega) \\
+B_{k+1}(\Omega)+\sigma_{k, 0}=0, \quad k>0
\end{gathered}
$$

where

$$
\langle\bar{v}\rangle=\left\langle\left(\theta_{\tau}-\theta\right) / \tau\right\rangle=\bar{i}+\operatorname{Re} \sigma_{1,0} .
$$

The latter expression gives the same result as (2.10). To get round avoidable singular point at $\Omega=0$ it is possible to transform the set $(2.40)$ to form

$$
\begin{gathered}
2[(\bar{i}+\Omega / k)-j k \Gamma] B_{k}^{\prime}(\Omega)+B_{k-1}^{\prime}(\Omega)+ \\
+B_{k+1}^{\prime}(\Omega)+(1-j<\bar{v}>/ k \Gamma) \sigma_{k, 0}=0, \\
k>0, \quad B_{0}^{\prime}(\Omega)=0,
\end{gathered}
$$


where $B_{k}^{\prime}=B_{k}+(j<\bar{v}>/ 2 \Gamma \Omega) \sigma_{k, 0}$. Thus to find spectral density $g(\Omega)$ it is necessary to solve the sets of linear eq. (2.38) and (2.42). The coefficient matricies of these sets of equations are three-diagonal. This allows to use special computer methods of calculation of $\sigma_{k, 0}$ and $B_{k}$.

Some of obtained results of calculations are shown by solid lines at figures $2 a$ and $2 b$. We have used such normalization procedure that curves at figures $2 a, 2 b$ correspond, for instance, to the variation of the spectral density of the voltage fluctuations at constant values of $R$ and $T$ with the variation of the critical current $I_{0}$. One can see how during increasing of the relative intensity of the fluctuations the lines of the Josephson oscillations are broadening and transform into usual thermal noise $(g=1)$.

When the bias is large $(\langle\bar{v}\rangle \widetilde{F} 1) \mathrm{g}$ is the sum of usual noise and Lorentz lines of the oscillations with bandwidths (2.29) practically at all values of $\Gamma$. The harmonics of the Josephson oscillations are overlapping at small values of $\langle\bar{v}\rangle$ and the spectrum shape is rather complicated. However when $\Gamma$ decreases the results obtained for small noise become valid.

The spectral density of the voltage fluctuations at low frequencies $s_{v}(0)(\Omega \ll\langle\bar{v}\rangle)$ and also in the vicinity of oscillations frequency

$$
s_{v}(<\bar{v}>+\Delta) \quad(\Delta=\Omega-\bar{v},|\Delta| \ll \Omega)
$$

has the particular meaning| for receiving devices. The dependence of $s_{v}(0)$ on bias voltage is shown at figure 3. The dotted line corresponds to (2.28). One can see that this expression can be used when condition

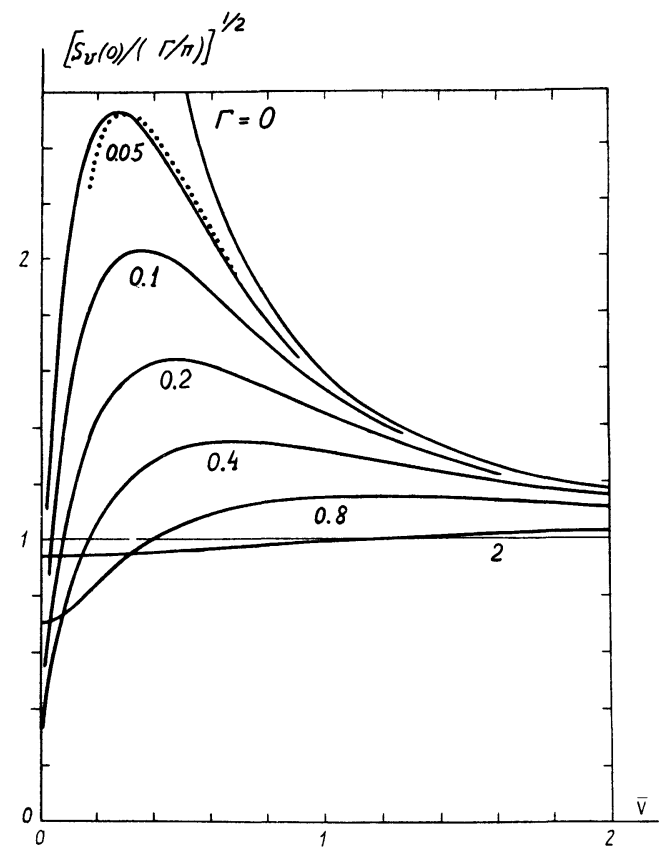

FIG. 3. - The spectral density of the fluctuations at low frequencies $(\Omega \ll \bar{v})$ as the function of the average voltage at different values of $\Gamma$. The dotted line corresponds to the result of the calculations at $\Gamma=0.05$ using expression (2.28). One can see that this expression can be used at $\bar{v} \widetilde{\sim} \Gamma^{1 / 3}$. of small value of noise (2.31) is fulfilled. We have for $s_{v}(\langle\bar{v}\rangle+\Delta)$ from (2.24) and (2.6) at small noise

$$
s_{v}(\Omega)=\frac{\left(\Gamma_{1} \ll \Omega\right)}{\pi\left(\Delta^{2}+\Gamma_{1}^{2}\right)(\bar{i} / \bar{v}+1)^{2}} .
$$

Unfortunately the experimental measurements of the noise properties of the S-c-S junctions were carried out at conditions when the low frequency noise voltage was shunted by measuring circuit [13] or bias circuit [51]. To verify the theoretical results obtained in this part it is desirable to carry out similar experiments more strictly.

3. The action of external signal on the junction. Linear effects. - 3.1 THE EQUIVALENT CIRCUIT OF THE JUNCTION. - We assume that the coupling current is small. Then we can try to obtain the solution of general equation for processes in the junction in the form $\varphi=\varphi^{(0)}+\varphi^{(1)}$, where $\varphi^{(0)}$ is the solution at $i_{\mathrm{e}}=0$ but with the fluctuation term, and $\varphi^{(1)}$ is small perturbation caused by the coupling current $\left(\varphi^{(1)} \sim i_{\mathrm{e}}\right)$. And then putting down $i_{\mathrm{e}}=i^{(1)}$ the linear eq. (2.13) is valid. The physical meaning of this equation is rather simple [52]. It is that the equivalent circuit of the junction for small signal consists of the parallel connection of the normal resistance $R$ and the kinetic inductance $L$

$$
1 / L=\left(1 / L_{0}\right) \cos \varphi^{(0)}, \quad L_{0}=\hbar / 2 \mathrm{e} I_{0}=R / \omega_{0} .
$$

This expression is valid both for $\mathrm{dc}\left(\varphi^{(0)}=\mathrm{Cte}\right)$ and for ac $\left(\varphi^{(0)}=\varphi^{(0)}(t)\right)$ Josephson effects. Thus the Josephson junction is the parametric element having two peculiarities.

(i) It can operate in the self-pumping regime [52]-

(ii) Its varying parameter $1 / L$ can change sign and moreover it has not dc component because

$$
\overline{\cos \varphi^{(0)}(t)=0} \quad \text { [52]. }
$$

These peculiarities result in the difference of the properties of the junction from the properties of usual parametric elements [44], [57]. The revealing themselves of these peculiarities depends essentialy on properties of a circuit into which the junction is inserted.

As it was mentioned in the introduction the signal acting on the nonlinear element can be expressed for the most of receiving devices in the form of sum of not large amount of quasi-harmonic components with the frequencies $\omega_{k}$. But such representation is valid either for voltage $V$ across the element or for current $I$ through the element dependingly on concrete system. These regimes correspond to the short circuit (sc) regime and the open circuit regime (oc) for combination frequencies. To realize them it is neces- 
sary to have the impedance $Z_{\mathrm{e}}$ of external circuit at the combination frequencies (of type $\omega_{\text {comb }}=\sum_{k} n_{k} \omega_{k}$ ), which are not included into set $\omega_{k}$ respectively, either much less or much higher than the characteristic impedance of the element (it is $R$ in our case). Existing now S-c-S junctions have values of the resistance from tenths to tens ohm. As for the impedances of the external microwave circuits they have values in range of hundreds ohm $\left({ }^{7}\right)$. Therefore the oc regime is only real regime at microwaves when $Z_{\mathrm{e}}$ is comparable with $R$ only at small amount of frequencies $\omega_{k}$ and $\left|Z_{\mathrm{e}}\right| \gg R$ at the rest of frequencies. In consequence of this fact results of calculations [52], [58], [59] published earlier are correct only in some special cases. Further we will consider everywhere only the oc regime, i. e. we will represent the coupling current $i_{\mathrm{e}}$ in the form of the sum of frequency components but not the voltage $v$ across the junction. Then we will find a response $v^{(1)}$ of the junction from the eq. (1.12) (in linear case from eq. (2.13)).

3.2 THE IMPedANCE OF THE JUNCTION. - Let fluctuations are absent at first, then the solution (2.17) and the Fourier-representation of $v^{(1)}(2.18)-(2.19)$ are valid. If the signal $\left(i_{\mathrm{e}}\right)$ of only one frequency $\Omega$ acts on the junction then $z_{0}(2.19)$ is normalized impedance $z_{0}=z=Z / R$ of the junction

$$
\begin{array}{r}
z=\operatorname{Re} z=1+\frac{1}{2(\bar{i}+\bar{v})}\left(\frac{1}{\bar{v}-\Omega}+\frac{1}{\bar{v}+\Omega}\right), \\
\bar{v}=\bar{v}^{(0)} \neq 0, \pm \Omega .
\end{array}
$$

$z$ has also imaginary part at the points $\bar{v}=0, \pm \Omega$. We have from (2.14) for superconducting region $(\bar{v}=0)$

$$
z=\left[1-j\left(1-\bar{i}^{2}\right)^{1 / 2} / \Omega\right]^{-1} .
$$

Using dispersion eq. [60] we obtain for $\bar{v}>0$

$$
\operatorname{Im} z=-\frac{\pi}{2} \cdot \frac{1}{\bar{i}+\bar{v}} \cdot \delta(\Omega-\bar{v}) .
$$

One can see from (3.2) that if the Josephson oscillations frequency $\bar{v}$ is some less than the signal frequency $\Omega$ then the impedance at microwaves can be negative. This fact was noted in [44], [57] and then in [59], [61], [62]. This phenomenon is absent in usual parametric systems in which an occurrence of negative resistance in the absence of exact coincidence of signal and pump frequencies is possible only in the presence of other frequency components [9]-[11].

The explanation of such " anomalous » impedance can be given in two ways and both versions being equivalent [57].

(7) One should bear in mind that inductance $L_{\mathrm{e}}$ of superconducting electrodes forming the junction is also connected in series with the external circuit. Usualy $\omega L_{\mathrm{e}}$ is also of order of hundreds ohm
Firstly such phenomenon will take place in any parametric element having peculiarity (ii) i. e. in which the parameter changes the sign. Indeed the equation of usual oscillator with varying parameters can be written in the form

$$
\ddot{x}+\omega_{0}^{2}(t) x=0
$$

from which one can see that if the parameter changes the sign then $\omega_{0}^{2}(t)<0$ and the eq. (3.5) describes exponential growth of variable $x$ but not stationary oscillations.

Secondly frequency relation $\bar{v} \approx \Omega$ corresponds to the proximity of the voltage to the lower edge of first step of the Josephson junction $i-v$ curve. As is known the location of the operating point at lower edge of this step corresponds to transfering of the energy from Josephson oscillations to the signal sinchronizing them. Therefore partial synchronization of Josephson oscillations will take place at $\bar{v} \approx \Omega$ also giving transfering of the energy of the same sign. However it is necessary to emphasize that anomalous impedance effect takes place in first approximation over signal, and Josephson step takes place in second approximation i. e. anomalous impedance reveals itself even at so small signal values when step is not forming yet.

We were not sure a priori is this effect stable with respect to fluctuations or not. More definitely it was not clear is intensity of fluctuations, at which the effect disappears, proportional to amplitude of the signal $i^{(1)}$ or not. In this case the effect would not exist at small signals.

To find $\langle z\rangle$ in the presence of intrinsic fluctuations the method was developed [38] allowing to find average value of any physical variable and being very similar to the method of finding the spectral densities used in the part $3\left({ }^{8}\right)$. According to this method we find the solution of the EFP equation at the presence of external signal

$$
\frac{\partial \sigma}{\partial t}=-\frac{\partial}{\partial \theta}\left[\sigma\left(\bar{i}+\cos \theta+i^{(1)} \cos \Omega t\right)\right]+\Gamma \frac{\partial^{2} \sigma}{\partial \theta^{2}}
$$

in the form of double Fourier series

$$
\begin{array}{r}
\sigma=\frac{1}{2 \pi} \sum_{k, k^{\prime}=-\infty}^{+\infty} \sigma_{k, k^{\prime}} \exp \left\{-j\left(k \theta+k^{\prime} \Omega t\right)\right\}, \\
\sigma_{k, k^{\prime}}=\sigma_{-k,-k^{\prime}}^{*}, \quad \sigma_{0, k^{\prime}}= \begin{cases}1, & k^{\prime}=0 \\
0, & k^{\prime} \neq 0 .\end{cases}
\end{array}
$$

We obtain from (3.6) two-dimensional infinite set of linear equations for coefficients $\sigma_{k, k^{\prime}}$

$$
\begin{aligned}
2(\bar{i}+ & \left.\frac{k^{\prime}}{k} \Omega-j k \Gamma\right) \sigma_{k, k^{\prime}}+\sigma_{k-1, k^{\prime}}+ \\
& +\sigma_{k+1, k^{\prime}}+i^{(1)}\left(\sigma_{k, k^{\prime}-1}+\sigma_{k, k^{\prime}+1}\right)=0 .
\end{aligned}
$$

(8) Independently similar method was developed in the work [63]. 
$\langle z\rangle$ can be expressed through $\sigma_{k, k^{\prime}}$ in following way

$$
<z>=1+\left(\sigma_{1,1}^{*}+\sigma_{1,-1}\right) / i^{(1)}
$$

and an expression for $\langle\bar{v}\rangle$ is given by (2.41) because coefficients $\sigma_{k, k^{\prime}}$ at $k^{\prime}=0$ coincide with values $\sigma_{k, 0}$ introduced in the part 2 .

During the investigation of the linear effects it is sufficient to consider three one-dimensional systems following from (3.8) at taking into account terms of first order over $i^{(1)}$. First of these systems is described by (2.38) and two others are described by following relation

$$
\begin{aligned}
2\left(\bar{i} \pm \frac{\Omega}{k}\right. & -j k \Gamma) \sigma_{k, \pm 1}+\sigma_{k-1, \pm 1} \\
& +\sigma_{k+1, \pm 1}+i^{(1)} \sigma_{k, 0}=0, k>0 .
\end{aligned}
$$

These equations differ from the eq. (2.42) for $B_{k}^{\prime}-s$ by free terms only and they can be solved by the same methods using computer. But it is seen even from (3.9) and (3.10) that $\langle z\rangle$ does not depend on $i^{(1)}$ at $i^{(1)} \rightarrow 0$.

Calculated in such manner dependences of $\langle z\rangle$ on $\langle\bar{v}\rangle$ [38] show that peculiarities of $\operatorname{Re} z$ and $\operatorname{Im} z$ at point $\bar{v}=\Omega$ are smoothing down. For instance we obtain immediately from (3.9) and (3.10) at $\Omega \gg 1$

$$
<z>=1+\frac{\Delta-j \Gamma}{4 \Omega\left(\Delta^{2}+\Gamma^{2}\right)},
$$

that with taking into account (2.29) shows that extrema of $\operatorname{Re}\langle z\rangle$ function have being reached at the deviation of the signal frequency a half of line bandwidth $(\Gamma)$ and

$$
\operatorname{Re}<z>_{\min }=1-\frac{1}{8 \Omega \Gamma} .
$$

Thus to obtain at some bias voltage a value of negative microwave impedance equal to $(\operatorname{Re} z)_{\min }$ the hf noise at $\Omega \gg 1$ should not exceed the value

$$
2 \Gamma=\frac{I_{\mathrm{fh}}}{I_{0}}=\frac{1}{4 \Omega\left[1-(\operatorname{Re} z)_{\min }\right]} .
$$

For arbitrary values of $\Omega$ the analytical expression for $z$ exists only in the region of small fluctuations

$$
\begin{gathered}
\left(\Gamma_{1} \ll \Omega\right) \\
<z>=1-\frac{1}{2(\bar{i}+\bar{v})} \cdot \frac{\Delta-j \Gamma_{1}}{\Delta^{2}+\Gamma_{1}^{2}}, \\
\Delta=\Omega-\bar{v}, \quad|\Delta| \ll \Omega,
\end{gathered}
$$

from which

$$
\frac{I_{\mathrm{fh}}}{I_{0}}=\frac{\bar{v}^{2}}{4(\bar{i}+\bar{v})\left(\bar{i}^{2}+1 / 2\right)\left[1-(\operatorname{Re} z)_{\min }\right]} .
$$

The values of $I_{\mathrm{fh}} / I_{0}$ for arbitrary $\Omega-\mathrm{s}$ are shown by solid lines at figure 4 , asymptotes at $\Omega \rightarrow \infty$ corresponding to relation (3.13). The similar dependences for the lf noise are shown by dashed lines at the same figure. One can make two conclusions from this figure.

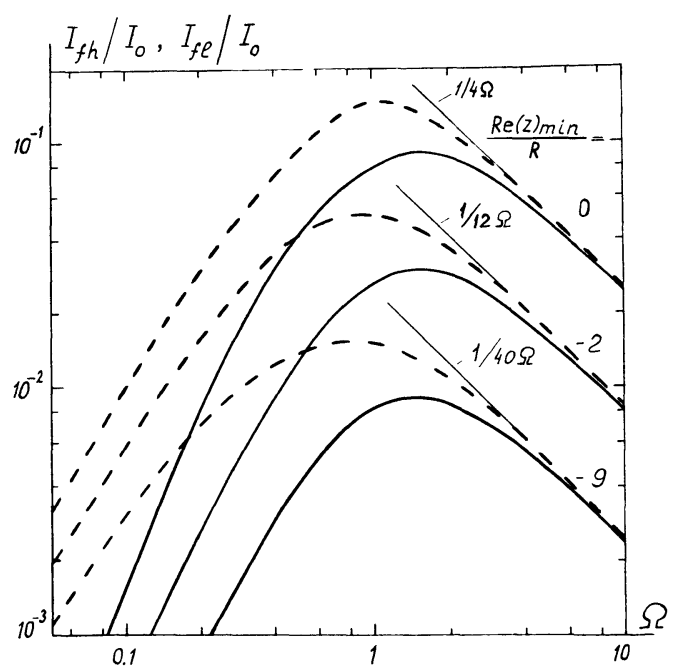

FIG. 4. - The value of the noise at which $(\operatorname{Re} z)_{\min }$ his given value. The dashed lines are for the lf noise, the solid lines are for the hf noise.

Firstly the action of If and hf fluctuations at $\Omega \sim 1$ is similar like in case of autonomous regimes (Fig. 1). It allows us in further analysis at $\Omega \widetilde{>} 1$ to calculate action of only hf fluctuations and to substitute simply $I_{\mathrm{f} 1}$ with $I_{\mathrm{fh}}$ in results in case $I_{\mathrm{f} 1} \gg I_{\mathrm{fh}}$.

Secondly the region of optimal values of frequency exists in which the noise influences on anomalous impedance effect $(\Omega \simeq 1)$ in the lowest rate.

We carried out [64] an experimental verification of the described phenomenon for the $\mathrm{Nb}-\mathrm{Nb}$ point contacts at $T=4.2 \mathrm{~K}\left({ }^{9}\right)$. To have a possibility to observe the effect of the variation of impedance but conserving at the same time the oc regime for all frequencies except signal frequency (X-band), the contact was inserted into central conductor of half-wavelength coaxial cavity. To have small influence of such cavity to Josephson oscillations process and to have therefore possibility to use above obtained relations, it is sufficient, as it will be shown later, to have the bandwidth of the cavity much less than oscillations line width. The cavity bandwidth in our experiments was about $20 \mathrm{MHz}$ while the oscillation line width was not less than $100 \mathrm{MHz}$. We have measured coefficient of reflection $|\gamma|^{2}$ of small microwave signal $\left(\approx 10^{-9} \mathrm{~W}\right)$ from the cavity connected to the termination of $\mathrm{X}$-band waveguide using the receiver $\prod^{5-10}$ having bandwidth $8 \mathrm{MHz}$. The coefficient $|\gamma|^{2}$ is

$$
|\gamma|^{2}=\left(P_{\mathrm{R}}-P_{0}\right) / P_{\mathrm{e}},
$$

(9) Somewhat later Kanter [65] has also observed anomalous impedance effect. 
where $P_{\mathrm{R}}$ is the reading on the scale of the receiver, $P_{\mathrm{e}}$ is the power of incident monochromatic signal, $P_{0}=P_{\mathrm{R}}$ if $P_{\mathrm{e}}=0$ is that portion of Josephson oscillations power which is contained in the bandwidth of the receiver. In the most of our experiments the relation $P_{0} \ll P_{\mathrm{R}}$ took place because of small bandwidth of the receiver. The signal frequency was tuned every time for resonance. In this case as is known

$$
\gamma=\frac{\operatorname{Re} z+r_{0}-r_{\mathrm{c}}}{\operatorname{Re} z+r_{0}+r_{\mathrm{c}}},
$$

where $r_{0}$ and $r_{\mathrm{c}}$ are reduced to the point of the contact and normalized over $R$ resistance of cavity losses and resistance of coupling of cavity with waveguide respectively $\left(r_{\mathrm{e}}=r_{0}+r_{\mathrm{c}}\right)$.

The dependences $|\gamma|^{2}$ and average voltage $\langle\bar{V}\rangle$ on the bias current $\bar{I}$ at two different levels of $P_{\mathrm{e}}$ for one of studed contacts $\left(R=(45 \pm 5) \times 10^{-3} \mathrm{ohm}\right)$ are shown at figure 5 . In this case the degree of the coupling of the cavity with the waveguide was chosen larger than critical $\left(r_{\mathrm{c}}>r_{0}\right)$ and therefore increasing of $|\gamma|^{2}$ corresponds to decreasing of $\operatorname{Re} z$. One can see that value of $\operatorname{Re} z$ at

$$
\bar{V} \simeq V_{\omega}=\frac{\hbar \omega}{2 \mathrm{e}} \simeq 19.2 \mu \mathrm{V}
$$

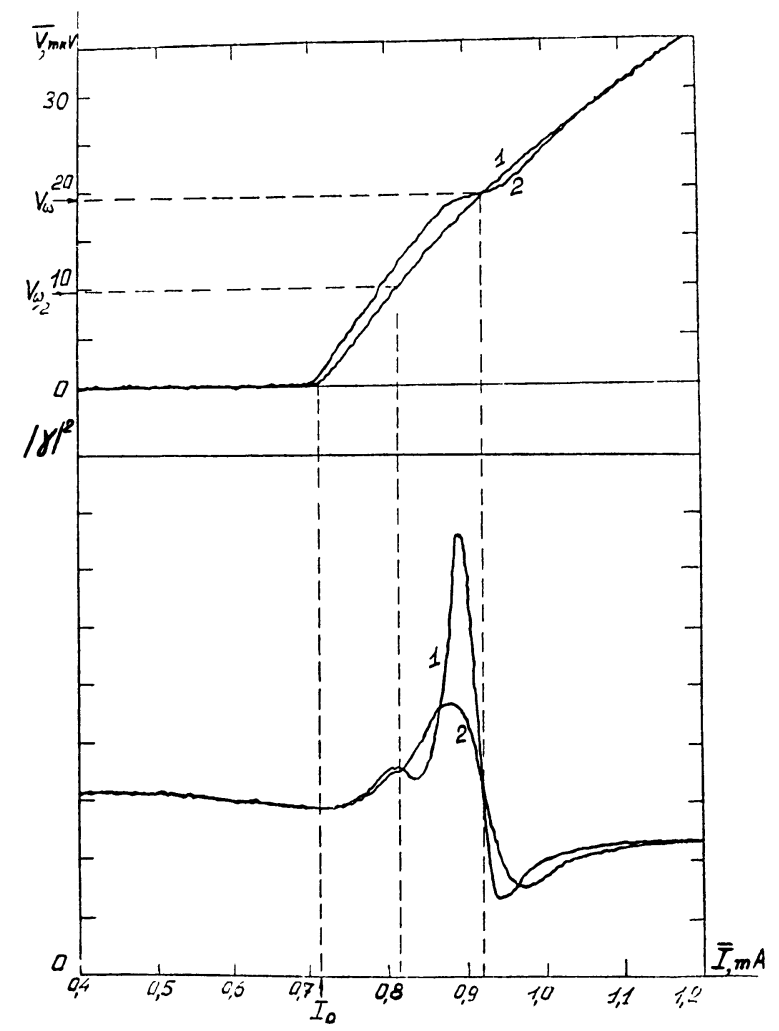

Fig. 5. - The dependences $|\gamma|^{2}$ and $\bar{V}$ on the bias current $\bar{I}$. The values of $P_{\mathrm{e}}$ are $8 \times 10^{-11} \mathrm{~W}(1)$ and $2.5 \times 10^{-9} \mathrm{~W}(2)$. One point at the axis of $|\gamma|^{2}$ corresponds to $0.1 \pm 0.03$. The value $\bar{V}=V_{\omega} \simeq 19.2 \mu \mathrm{V}$ corresponds to the equality of frequencies of the oscillations and the signal $(\bar{v}=\Omega)$. has a peculiarity the shape of which corresponds to theoretically predicted one with taking into account fluctuations. For instance the calculation for the curve 1 using (3.15) gives $(\operatorname{Re} z)_{\min }=-1.7 \pm 0.5$ while the theory gives $(\operatorname{Re} z)_{\min }=-3 \pm 1$. The errors of experiment are connected mainly with non-accuracy of finding $r_{0}$ and $r_{\mathrm{c}}$. The finding $r_{0}$ and $r_{\mathrm{c}}$ from (3.15) is complicated by the fact that $\operatorname{Re} z>0$ even at the current less than critical current (3.3). The error of calculated value of $(\operatorname{Re} z)_{\min }$ is connected with non-accuracy of determining parameters of the resistive model $(\Omega=0.6 \pm 0.1$ and $\left.I_{\mathrm{f} 1} / I_{0}=(34 \pm 4) \times 10^{-3}\right)$ from the experimental data.

We have observed anomalous impedance in every studied contact for which we could achieve required small value of external interferences (Fig. 4). The effect for low resistance contacts $\left(R \approx 10^{-2} \mathrm{ohm}\right)$, characteristics of which are very different from ones following from resistive model, was also observed though its value was essentially less than it follows from the theory. Besides impedance peculiarities of low resistance contacts reveal themselves distinctly at voltages $\bar{V} \simeq V_{\omega} / n$ like at the fundamental voltage. We have observed them at $n=2,3,4$. These additional peculiarities are explained easily by interaction of signal with harmonics of Josephson oscillations, contribution of which increases at decreasing of $\mathrm{R}$. Revealing itself of peculiarity at $n=2$ is observable at figure 4.

If $\operatorname{Re} z<-r_{0}$ at some bias then net amplification of incident microwave signal takes place in the cavity. We have observed such amplification for several decibels. Theoretical estimations of possible parameters of such amplification are given below.

If more strong condition

$$
\operatorname{Re} z<-\left(r_{0}+r_{\mathrm{c}}\right)=-r_{\mathrm{e}}
$$

is satisfied then oscillations should be excited in the cavity at own frequency different from the Josephson frequency. As the description of the process using linear theory is not applicable, the investigation of this question is carried out in the part 5 .

A saturation of effect i. e. decreasing of variation of $|\gamma|^{2}$ is observed (Fig. 5, curve 2) at increasing of incident power $P_{\mathrm{e}}$ higher than some value $P_{\mathrm{s}}$. The forming of first step of the $i-v$ curve of the Josephson junction (Fig. 5) takes place exactly at values of signal power of order of $\simeq P_{\mathrm{s}}$ and this is in accordance with results which can be obtained from (2.41), (3.8) and (3.9).

Several theoretical dependences $z(\bar{I})$ for large values of signal are given in [62]. Russer and Bayegan [90] have concluded that the parametric regeneration is impossible because of studying processes in the junction at large amplitudes of the signal.

3.3 The PARAMETRIC CONVERSION EFFICIENCY. The expression (2.19) for the parametric conversion 
factors shows clearly that anomalous behaviour of the impedance $\left(z_{0}\right)$ is not isolated phenomenon and all coefficients $z_{k}$ contain similar terms. Let us consider for instance the case when frequency of signal $\Omega$ and operating frequency of receiver $(\Omega+\Delta)$ are not high and are almost equal one another.

$$
|\Delta| \ll \Omega \ll 1 \text {. }
$$

Then according to $(2.20)$ combination frequencies will be received by the receiver at the condition $\pm \Omega \simeq \Omega-k \bar{v}_{k}$ i. e. at

$$
\bar{v}_{n} \simeq \frac{2 \Omega}{n}, \quad n>0 \text {. }
$$

Such conversion also takes place for usual parametric element, value of signal being proportional to the amplitude of $n$th harmonic of parameter variation function. Since $1 / L \sim \cos \varphi^{(0)}(t)$ in our case it is easy to obtain from (2.3) that amplitudes of the harmonics decrease monotonously with increasing of $n$. Therefore the values of $\left|z_{n}\right|$ would decrease monotonously with increasing of $n$ if the S-c-S junction was usual parametric element. However we have from (2.19) for this case

$$
\left|z_{n}\right|=\frac{6}{\Omega}\left|\frac{n}{n^{2}-4}\right|, \quad n \neq 2, \quad\left|z_{2}\right|=\frac{1}{|\Delta|} \text {. }
$$

Thus coefficient $z_{2}$ is anomalously large i. e. the combination frequency corresponding to $k=2$ (at $\left.\bar{v} \simeq \Omega, \bar{V} \simeq V_{\omega}\right)$ should increase more rapidly than others at the increasing of input signal $\left(i^{(1)}\right)$.

We have observed this effect experimentally [64] during the investigation of the negative impedance effect. To observe the combination effects the frequency of the external signal was shifted a little ( $\sim 20 \mathrm{MHz}$ ) from receiver operating frequency. The dependences of the power $P_{\mathrm{R}}$ received by the receiver on $\bar{V}$ at different values of incident signal $P_{\mathrm{e}}$ are shown at figure 6 . The line bandwidth of combination

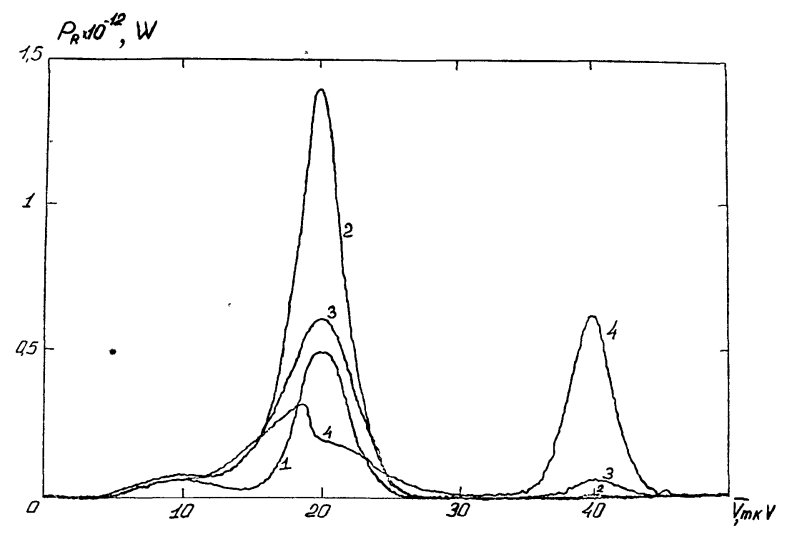

FIG. 6. - The radiation at combination frequencies from the same junction that is at figure 5. The curve I shows the level of the Josephson oscillations $\left(P_{\mathrm{e}}=0\right)$. For the rest of the curves $P_{\mathrm{e}}$ is equal to $2 \times 10^{-9} \mathrm{~W}(2) 6 \times 10^{-9} \mathrm{~W}(3)$ and

$$
2 \times 10^{-8} \mathrm{~W}(4) \text {. }
$$

frequency components is determined by the bandwidth of oscillations line. It is seen that the conversion efficiency is maximum at $n=2$ according to the theory at small values of $\boldsymbol{P}_{\mathrm{e}}$ (linear theory).

The experimental observation of the peculiarities of the S-c-S junctions with self-pumping corroborates very well the equivalent circuit (3.1) and shows that the theory of parametric systems on the basis of the S-c-S junctions in the oc regime should be developed using this equivalent circuit and usual theory [9]-[11] cannot be used. Using this equivalent circuit one can calculate the characteristics including noise characteristics of any parametric system using S-c-S junction with self-pumping.

We have restricted our consideration by two systems which are interesting from practical point of view.

(i) Down-converters to frequency $\left({ }^{10}\right)$ much less than signal frequency.

(ii) One-frequency negative resistance amplifiers.

3.4 THE WIDE-BAND PARAMETRIC DOWN-CONVERTER WITH SELF-PUMPING. - According to the general expression (2.19) for conversion factors the maximum conversion efficiency of signal at frequency $\Omega$ to signal at frequency $|\Delta| \ll \Omega$ (we assume also that $|\Delta| \ll 1$ that is valid practically for any S-c-S junctions at frequencies even up to $X$-band range) is being achieved when $k=1 \mathrm{i}$. e. when the signal with frequency which is the difference between frequency of input signal and frequency of Josephson oscillations

$$
\Delta=\Omega-\bar{v} \quad(\Delta \lessgtr 0)
$$

is used as output signal.

As it was shown earlier intensive producing of the component with image frequency

$$
2 \bar{v}-\Omega=\bar{v}-\Delta \simeq \bar{v},
$$

besides usual pair of components with combination frequencies $|\Omega \pm \bar{v}|$, takes place in the junction at such regime.

As $|\Delta| \ll \bar{v}, \Omega$ this component can be in the bandwidth of external microwave circuit into which the junction is inserted if this bandwidth $h_{\mathrm{h}}$ is sufficiently large $\left({ }^{11}\right)$

$$
h_{\mathrm{h}} \gg|\Delta|, \Gamma_{1} .
$$

We will call this regime a wide-band regime and will analize it in the first place.

Let the influence of fluctuations is small at first. Then according to (2.19) and using inequality

(10) We shall call this frequency a «low frequency» for convenience although it could be rather high in real receiving devices.

(11) Of course the line bandwidths of all combination components are of order of oscillations line bandwidth. 
$|\Delta| \ll 1 \ll \Omega$ we write down the junction impedance matrix for main frequency triplet $(\Delta \pm \bar{v}, \Delta)\left({ }^{12}\right)$

$|z|=\left[\begin{array}{ccc}1-\frac{1}{2 \Delta(\bar{i}+\bar{v})} & -\frac{1}{2 \Delta(\bar{i}+\bar{v})} & \frac{\bar{i}}{(\bar{i}+\bar{v}) \Delta} \\ \frac{1}{2 \Delta(\bar{i}+\bar{v})} & 1+\frac{1}{2 \Delta(\bar{i}+\bar{v})} & \frac{-\bar{i}}{(\bar{i}+\bar{v}) \Delta} \\ -\frac{1}{2 \vec{v}} & -\frac{1}{2 \bar{v}} & \frac{\bar{i}}{\bar{v}}\end{array}\right]$.

Let external circuit be tuned in resonance both at input $(\bar{v} \pm \Delta)$ and at output $(|\Delta|)$ frequencies. Then to obtain total conversion matrix it is sufficient to add normalized active resistances of the circuit at hf $\left(r_{\mathrm{h}}\right)$ and at If $\left(r_{1}\right)$ to diagonal terms of matrix (3.23). Assuming that losses of the circuit at hf and lf are small so that $r_{\mathrm{h}}$ and $r_{1}$ are signal source and load resistances respectively reduced to the point of the junction we obtain in usual way [9]-[11] power conversion factor

$$
G=\alpha_{1} \alpha_{\mathrm{h}} / 16 \Omega^{2} .
$$

Matching factors $\alpha_{\mathrm{h}}$ and $\alpha_{1}$ at high and low frequencies are

$$
\begin{aligned}
\alpha_{\mathrm{h}} & =\frac{4 r_{\mathrm{h}}}{\left(1+r_{\mathrm{h}}\right)^{2}} \leqslant 1, \\
\alpha_{1} & =\frac{4 r_{1} r_{\mathrm{d}}}{\left(r_{1}+r_{\mathrm{d}}\right)^{2}} \leqslant 1, \\
r_{\mathrm{d}} & =\frac{\bar{i}}{\bar{v}} .
\end{aligned}
$$

The expressions (3.24), (3.25) show that effective input and output resistances of the down-converter are $R$ and $R_{\mathrm{d}}$ and that $G$ does not depend on low frequency $(\Delta)\left({ }^{13}\right)$. Thus the anomalous impedance effect does not give a contribution to conversion factor at wide-band regime. As it is seen from matrix (3.23) it takes place because anomalous contribution of energy to the component at frequency $\bar{v}+|\Delta|$ is compensated by similar extraction of energy from the component at frequency $\bar{v}-|\Delta|$.

One can understand now that if fluctuations in the circuit are not too large so that a condition for the bandwidth of the If circuit

$$
h_{1} \gg \Gamma_{1}
$$

is fulfilled besides fulfillment of condition (3.22) then the result (3.24) is valid again. And if the relation

(12) Assuming that the external circuit impedance at fourth essential frequency $(\bar{v}+\Omega=2 \bar{v}+\Delta \simeq 2 \bar{v})$ is large.

(13) The similar result was obtained also in [66].
(3.26) is not fulfilled then only a portion of converted signal passes through the if circuit.

Let now if and hf impedances are choosen correctly $\left(r_{1}=r_{\mathrm{d}}, r_{\mathrm{h}}=1\right)\left({ }^{14}\right)$. Then

$$
G=\left(16 \Omega^{2}\right)^{-1} .
$$

It is easy to explain this result. The anomalous terms in $z_{k}$ at three-frequency regime are mutually compensating and the S-c-S junction has properties similar to usual parametric element with critical frequency $\omega_{\mathrm{c}}=\omega_{0} / 2$ [44], [52]. Therefore expression (3.27) can be rewritten in the form

$$
G_{\max }=\frac{1}{4}\left(\frac{\omega_{\mathrm{c}}}{\omega}\right)^{2}
$$

which is usual result for down-converter [10], [11]. It was used $\omega_{0}$ instead of $\omega_{c}$ in corresponding expression by mistake in the work [58].

The noise properties of such down-converter can be also determined easily. Using usual method [9]-[11] with taking into account equalities (2.28) and (2.43) we obtain for noise temperature reduced to the input $\left(\alpha_{\mathrm{h}}=1\right)$ in the most interesting case of narrow line bandwidth $\left(\Gamma_{1} \ll|\Delta|\right)$

$$
\begin{gathered}
\frac{T_{\mathrm{in}}}{T}=2 \Omega^{2} \frac{\left(1+r_{\mathrm{h}}\right)^{2}}{r_{\mathrm{h}}}, \\
\left(\frac{T_{\mathrm{in}}}{T}\right)_{\min }=8 \Omega^{2} .
\end{gathered}
$$

The down-conversion with self-pumping was observed qualitatively in works [67], [68] and also in our experiments (from four-millimeter waveband range to $60 \mathrm{MHz}$ frequency range). The minimum conversion loss equal to $11 \mathrm{db}$ was obtained in the work [58] during the conversion from $34 \mathrm{GHz}$ to $300 \mathrm{MHz}$ frequency range using $\mathrm{NbZr}-\mathrm{Nb}$ point contacts at $V_{0} \simeq 300 \mu \mathrm{V}(\Omega \simeq 1 / 4)$. According to (3.28) the theoretical value of conversion loss for this case is $\left(G^{-1}\right)_{\min } \simeq 0 \mathrm{~dB}\left({ }^{15}\right)$. The discrepancy may be connected with matching losses $\left(\alpha_{\mathrm{h}, 1}<1\right)$ and passing through narrow-band if circuit $(30 \mathrm{MHz})$ only portion of converted signal. So the conversion with self-pumping was not observed at all in the work [66] because oscillations line bandwidth caused by external interferences was about $1 \mathrm{GHz}$. The experimental investigation of self-pumping at low frequencies was carried out also in the work [107].

(14) The correspondence of optimal regime to condition $r_{\mathrm{h}}=1$ proves our tacit assumption that $\mathrm{hf}$ bandwidth of external circuit does not influe on Josephson oscillations process and therefore on the matrix (3.23). As it will be shown in the part 5 this assumption at $\Omega \widetilde{>} 1$ is valid always and is valid only when $r_{\mathrm{h}} \approx \Omega \ll 1$ at $\Omega \ll 1$

(15) One can see that this effect is not connected with the presence of the anomalous impedance. 
3.5 The NegATIVE RESISTANCE AMPLIFIER. - It is natural to try to use anomalous impedance effect in the S-c-S junctions for constructing one-frequency hf amplifiers and improving efficiency of the downconversion. As it was shown above it is necessary for this to prevent the passing of the image frequency component $(\bar{v}-\Delta)$ to the hf bandwidth of external circuit. As it is easy to understand it is fulfilled at

$$
h_{\mathrm{h}} \approx \max \left[\Gamma_{1},|\Delta|\right] \text {, }
$$

i. e. at sufficiently narrow bandwidth of hf circuit (cavity). At the fulfillment of this condition the influence of the cavity on the Josephson oscillations process is small and we can use obtained above expressions (3.2) for the junction impedance for one-frequency regime (the impedance of external circuit at low frequency $\sim|\boldsymbol{\Delta}|$ is large in comparison with $r_{\mathrm{d}}$ ).

As is well-known the gain $G$ of negative resistance amplifier can be made arbitrarily large if one can make $|\operatorname{Re} z|$ almost equal to reduced to the junction impedance $r_{\mathrm{h}}$ of hf circuit (the cavity losses are assumed to be small, $r_{\mathrm{h}} \simeq r_{\mathrm{c}}$ )

$$
\operatorname{Re} z+r_{\mathrm{h}}=0
$$

at fixed efficiency

$$
\varepsilon \equiv G^{1 / 2} \frac{2 \tilde{h}_{\mathrm{h}}}{\Omega}=\frac{2 r_{\mathrm{h}}}{\rho_{\mathrm{h}}},
$$

where $\tilde{h}_{\mathrm{h}}$ is half of the bandwidth of regenerated cavity with own frequency $\Omega_{\mathrm{h}} \simeq \Omega, \rho_{\mathrm{h}}$ is its wave resistance. We have for the noise temperature reduced to the input at strong regeneration using its definition given in [9]-[11]

$$
\frac{T_{\mathrm{in}}}{T}=\frac{s_{v}(\Omega)}{\left[s_{v}(\Omega)\right]_{r_{\mathrm{h}}}}=\frac{g(\Omega)}{r_{\mathrm{h}}} .
$$

Since negative impedance reveals itself most intensively at the small noise $\left(\Gamma_{1} \ll \Omega\right)$ we restrict our consideration by this case. Substituting values of $\operatorname{Re} z$ (3.14) to (3.32) and (3.33) we have at optimal bias $\left(\Delta=\Gamma_{1}\right)$

$$
\varepsilon=\frac{2(Q-1)}{\rho_{\mathrm{h}}}
$$

The parameter

$$
\begin{aligned}
Q & =\frac{1}{4 \Gamma_{1}(\bar{i}+\bar{v})} \simeq \frac{1}{4 \Gamma_{1}\left[\left(1+\Omega^{2}\right)^{1 / 2}+\Omega\right]} \\
& = \begin{cases}(8 \Omega \Gamma)^{-1}, & \Omega \gg 1 \\
\Omega^{2} / 6 \Gamma, & \Omega \ll 1\end{cases}
\end{aligned}
$$

defines the possibility of regeneration at given frequency and at given level of fluctuations as for this device so for other devices. If $Q<1$, the regenerative amplification is impossible. $\Omega_{\mathrm{opt}} \simeq 1.1$ at fixed level of fluctuations. Besides choosing optimal $\Omega$ one can to improve efficiency decreasing $\rho_{\mathrm{h}}$. A possibility of decreasing $\rho_{\mathrm{h}}$ is limited by the presence of inductance $L_{\mathrm{e}}$ of electrodes forming the junction

$$
\left(\rho_{\mathrm{h}}\right)_{\min } \simeq \frac{\omega L_{\mathrm{e}}}{R}
$$

The optimizing $\varepsilon$ (i. e. obtaining the equalities $\left.\Delta=\Gamma_{1}, \quad r_{\mathrm{h}}=Q-1\right)$ is necessary when noise of following stages of the receiver is large. If noise is small then the optimization of amplifier consists in the minimization of its noise temperature $T_{\mathrm{in}}$. We obtain from (3.34) and (2.43) in this case for $\Omega\left\ulcorner 1\left({ }^{16}\right)\right.$ at $\Delta=(8 \bar{i})^{-1}$ and $r_{\mathrm{h}}=1$

$$
T_{\text {in }}=16 \Omega^{2}\left(1-Q^{-2}\right)^{-1} T
$$

which coincides with corresponding expression for wide-band (non-regenerative) converter at $Q \gg 1$.

If the bias is chosen to achieve maximum $\varepsilon(3.35)$ then

$$
T_{\text {in }}=8\left(\Omega^{2}+\frac{3}{2}\right)\left(Q^{-1}-Q^{-2}\right)^{-1} T
$$

and $T_{\text {in }} \simeq 2\left(T_{\text {in }}\right)_{\min }$ at $\Omega \gtrsim 1$ if $Q=2$.

3.6 THE REgenERATIVE DOWN-CONVERTER. - Let now add the if circuit at frequency $|\Delta|$ with bandwith $h_{1} \sim \Gamma_{1}$ to the regenerative circuit and take output signal from this circuit. We have now down-converter regenerated over high frequency circuit. We consider again only case when $\Delta>0$ because in opposite case the real part of the anomalous hf impedance of the junction is positive and conversion efficiency is low [44], [57], [69]. It is interesting to note that the case $\Delta=\Omega-\bar{v}<0$ is on the contrary regenerative for usual parametric elements [9]-[11].

Since image frequency $(\bar{v}-\Delta)$ is not in the bandwidth of hf circuit one can use for the analysis matrix (3.23) crossing out second row and second column from it. Taking into account the influence of fluctuations we obtain at the resonance frequency

$$
G=\frac{r_{1} r_{\mathrm{h}}}{\bar{v}^{2}\left(r_{\mathrm{d}}+r_{1}\right)^{2}\left[r_{\mathrm{h}}+1-\frac{r_{1}}{r_{\mathrm{d}}+r_{1}} \cdot \frac{\Delta}{2(\bar{i}+\bar{v})\left(\Delta^{2}+\Gamma_{1}^{2}\right)}\right]^{2}} .
$$

The expression in brackets is the effective hf impedance of converter. It is seen that the transfering of energy to if circuit $\left(r_{1} \neq \infty\right)$ decreases anomalous

(16) The calculation for $\Omega \approx 1$ is more complicated. Its results will be published later. 
part of this impedance in some degree. In the case of strong regeneration we have for the efficiency

$$
\varepsilon=\frac{\left(r_{1} r_{\mathrm{h}}\right)^{1 / 2}}{\bar{v}\left(r_{\mathrm{d}}+r_{\mathrm{l}}\right) \rho_{\mathrm{h}}}
$$

at the condition for $r_{\mathrm{h}}$

$$
r_{\mathrm{h}}+1 \simeq \frac{r_{1}}{\left(r_{\mathrm{d}}+r_{1}\right)} \cdot \frac{\Delta}{2(\bar{i}+\bar{v})\left(\Delta^{2}+\Gamma_{1}^{2}\right)} .
$$

Assuming that $Q \gg 1$ and $|\Delta|$ is fixed we obtain maximum efficiency at $r_{1}=2 r_{\mathrm{d}}, r_{\mathrm{h}}=(2 / 3) Q \gg 1$

$$
\varepsilon_{\max }=\frac{1}{3 \sqrt{3}} \cdot \frac{|\Delta|^{-1}}{\Omega \rho_{\mathrm{h}} r_{\mathrm{d}}^{1 / 2}} .
$$

The noise temperature of the converter reduced to input $\left(T_{\mathrm{in}}\right)$ at the sufficiently strong regeneration is the same as for the amplifier and reaches the same minimum value (3.38) at additional condition $r_{1} \sim r_{\mathrm{d}}$.

4. The action of external signal on the junction. Quadratic effects. - 4.1 THE RESPONSE OF THE JUNCTION TO HARMONIC SIGNAL. - Let small harmonic current $\left(i_{\mathrm{e}} \ll 1\right)$ of normalized frequency $\Omega$ flows through the S-c-S junction. The most interesting and important effect which value is proportional to the square of amplitude $\left(i^{(1)}\right)$ of this current is the variation (" response ") of the $i-v$ characteristic of the junction under influence of this current. This phenomenon is using for constructing the Josephson detectors [43], [61], [70]-[75].

For the case of the absence of the noise the variation $\tilde{\bar{i}}$ of average current through the junction is

$$
\begin{aligned}
\tilde{i} & =\left(i^{(1)}\right)^{2} / 4 \bar{i}\left(\bar{v}^{2}-\Omega^{2}\right), \\
\bar{v} & =\left(\bar{i}^{2}-1\right)^{1 / 2}>0 .
\end{aligned}
$$

This expression was obtained by Kanter and Vernon [61] and for special cases $\bar{v} \ll \Omega$ and $\bar{v} \gg 1$ even earlier in [16] and [76] respectively. We will give here the derivation of the expression (4.1) more brief than in [61].

As the basis we will use the eq. (1.12) in the absence of fluctuations

$$
\dot{\varphi}+\sin \varphi=i+i^{(1)} \sin \Omega t
$$

and will find its solution in the form

$$
\varphi=\varphi^{(0)}+\varphi^{(1)}+\varphi^{(2)}, \varphi^{(k)} \sim\left(i^{(1)}\right)^{k} .
$$

Using (4.2) we obtain the set of equations

$$
\begin{aligned}
\dot{\varphi}^{(0)}+\sin \varphi^{(0)} & =\bar{i}-\tilde{\bar{i}} \\
\dot{\varphi}^{(1)}+\cos \varphi^{(0)} \cdot \varphi^{(1)} & =i^{(1)} \sin \Omega t \\
\dot{\varphi}^{(2)}+\cos \varphi^{(0)} \cdot \varphi^{(2)} & =\widetilde{\bar{i}}+\sin \varphi^{(0)} \cdot\left(\varphi^{(1)}\right)^{2} / 2 .
\end{aligned}
$$

Unknown quantity $\tilde{\bar{i}}$ should be chosen so that $\varphi^{(2)}$ having not divergent term $\left(\left|\varphi^{(2)}\right| \ll 1\right)$. As it follows from (2.17) it is necessary for this that right-hand side of eq. (2.17) devided by $\dot{\varphi}^{(0)}$ having not dc component. We obtain from this requirement

$$
\begin{aligned}
\tilde{\bar{i}} & =-\frac{1}{2 \bar{i}} \overline{(\bar{i}-\cos \bar{v} t) \sin \varphi^{(0)} \cdot\left(\varphi^{(1)}\right)^{2}} \\
& =-\frac{1}{2 \bar{i}} \overline{(1-\bar{i} \cos \bar{v} t)\left(\varphi^{(1)}\right)^{2}} .
\end{aligned}
$$

Finding $\varphi^{(1)}$ from $(4.3 b)$

$$
\begin{gathered}
\varphi^{(1)}=\frac{i^{(1)}}{\bar{i}-\cos \bar{v}} \cdot\left\{-\frac{\bar{i}}{\Omega} \cos \Omega t+\right. \\
\left.+\frac{1}{2(\Omega-\bar{v})} \cos (\Omega-\bar{v}) t+\frac{1}{2(\bar{v}+\Omega)} \cos (\Omega+\bar{v}) t\right\} .
\end{gathered}
$$

and taking into account the equality

$$
\frac{1-\bar{i} \cos \bar{v} t}{(\bar{i}-\cos \bar{v} t)^{2}}=-2 \sum_{k=1}^{\infty} \frac{k \cos k \bar{v} t}{(\bar{i}+\bar{v})^{k}},
$$

we obtain that only terms of $\left(\varphi^{(1)}\right)^{2}$ containing $\cos \bar{v} t$ and $\cos 2 \bar{v} t$ should be taken into account

$\left(\varphi^{(1)}\right)^{2} \rightarrow \frac{\left(i^{(1)}\right)^{2}}{(\bar{i}-\cos \bar{v} t)^{2}}\left[-\frac{\bar{i} \cos \bar{v} t}{\Omega^{2}-\bar{v}^{2}}+\frac{\cos 2 \bar{v} t}{4\left(\Omega^{2}-\bar{v}^{2}\right)}\right]$.

Substituting this expression to (4.4) and carrying out averaging process we obtain Kanter-Vernon formula.

According to (4.1) two regions of "wide-band " detection exist : one at $\bar{v} \widetilde{>} \Omega$ and another at $\bar{v} \approx \Omega$. In this regions the response of the junction is connected with total variation of $i-v$ curve and changes relatively slowly at variation of signal frequency. First of these regions is "classic " in the sense that the voltage response does not depend on signal frequency and is proportional to the curvature of $i-v$ curve

$$
\tilde{\bar{v}} \equiv-r_{\mathrm{d}} \cdot \tilde{\bar{i}}=\frac{\left(i^{(1)}\right)^{2}}{4} \cdot \frac{\mathrm{d}^{2} \bar{v}}{\mathrm{~d} \bar{i}^{2}}, \quad r_{\mathrm{d}}=\frac{\mathrm{d} \bar{v}}{\mathrm{~d} i} .
$$

The characteristic time of reaching the steadystate Josephson oscillations in the junction $\left(\sim \bar{v}^{-1}\right)$ in the second of these regions is larger than period of signal variation $2 \pi \Omega^{-1}$. It results in the response decreasing when signal frequency increases

$$
\tilde{\bar{v}}=r_{\mathrm{d}}\left(i^{(1)}\right)^{2} / 4 \bar{i} \Omega^{2} \sim \Omega^{-2} .
$$

Two mentioned regions are separated by the region of selective detecting where $\bar{v} \simeq \Omega$. The response in this region is connected with the beginning of forming of the first Josephson current step at the $i-v$ curve and as it is seen from (4.1) its dependence on $\Omega$ is resonant. 
In the presence of fluctuations the value of the response is no longer describing by (4.1). It is useful here again to consider small and large fluctuations (see the part 2). At small fluctuations the expression (4.1) is valid for all frequencies except small vicinity of point $\Omega=\bar{v}$ with bandwidth $\sim \Gamma_{1}$. The taking into account even small fluctuations in this vicinity results in vanishing of the divergence of the response at $\Omega=\bar{v}$ i. e. in proportionality of appearing first Josephson step not to the signal amplitude but to its square

$$
\begin{aligned}
\tilde{\bar{v}}=\left(i^{(1)}\right)^{2} \cdot \frac{\Delta}{8 \Omega^{2}\left(\Delta^{2}+\Gamma_{1}^{2}\right)}, \\
\quad \Delta=\Omega-\bar{v}, \quad \Gamma_{1},|\Delta| \ll \Omega .
\end{aligned}
$$

As it is seen from the comparison of (4.1) and (4.10) the peak of selective response at $\Gamma_{1} \sim \Omega$ is smoothed down completely. For the case $\Omega \sim 1$ this expression was obtained at the works [35], [63], [77], [78].

As it will be shown later, to find limit parameters of detectors using the S-c-S junctions it is also necessary to know a value of the response at large fluctuations when the expression (4.1) is invalid completely. We will apply in this case the EFP equation method described in the part III. Namely saving second order terms in respect to $\left(i^{(1)}\right)$ in the set of eq. (3.8) we obtain the set of equations for correction terms $\sigma_{k, 0}^{(2)}$ to quantities $\sigma_{k, 0}$

$$
\begin{aligned}
& 2(\bar{i}-j k \Gamma) \sigma_{k, 0}^{(2)}+\sigma_{k-1,0}^{(2)}+\sigma_{k+1,0}^{(2)}+ \\
& +i^{(1)}\left(\sigma_{k,-1}+\sigma_{k, 1}\right)=0, \quad \sigma_{0,0}^{(2)}=0, \quad k>1,
\end{aligned}
$$

where values $\sigma_{k, \pm 1}$ satisfy the eq. (3.10). At such consideration the response of detector

$$
\tilde{\bar{v}}=\operatorname{Re} \sigma_{1,0}^{(2)} \text {. }
$$

The dependence of the value $\eta$ proportional to the response (see below) on the bias voltage at different levels of noise $(\Gamma)$ is shown at figure 7 as an example. The smoothing down of the peak of the selective detection and complete invalidity of the expression (4.1) at $\Gamma \widetilde{>} 1$ are seen well.

4.2 THE JOSEPHSON DETECTOR IN THE WIDE-BAND REGIME. - Let the junction is inserted into external microwave circuit having the impedance $z_{\mathrm{e}}$ with respect to the junction and coupling impedance $r_{\mathrm{c}}$ reduced to the junction $\left(r_{\mathrm{c}} \leqslant \operatorname{Re} z_{\mathrm{e}}\right)$. Let further fundamental harmonics lines of Josephson oscillations of the junction and also lines of main combination frequencies $\bar{v} \pm \Omega$ and $2 \bar{v}-\Omega$ are not in the bandwidth of the circuit i. e.

$$
\left|z_{\mathrm{e}}\right| \gg 1
$$

at these frequencies.
These assumptions are fulfilled unconductionally for instance at sufficiently narrow bandwidth of the circuit $\left(h_{\mathrm{h}} \approx \Gamma_{1}\right)$. Moreover mentioned conditions are not fulfilled only in one case when the junction has low resistance $\left(\Gamma_{1} \ll 1\right)$, bias voltage is $\bar{v} \simeq \Omega$ and the bandwidth of the circuit is large, i. e. in selective regime. Therefore the selective regime will be considered especially.

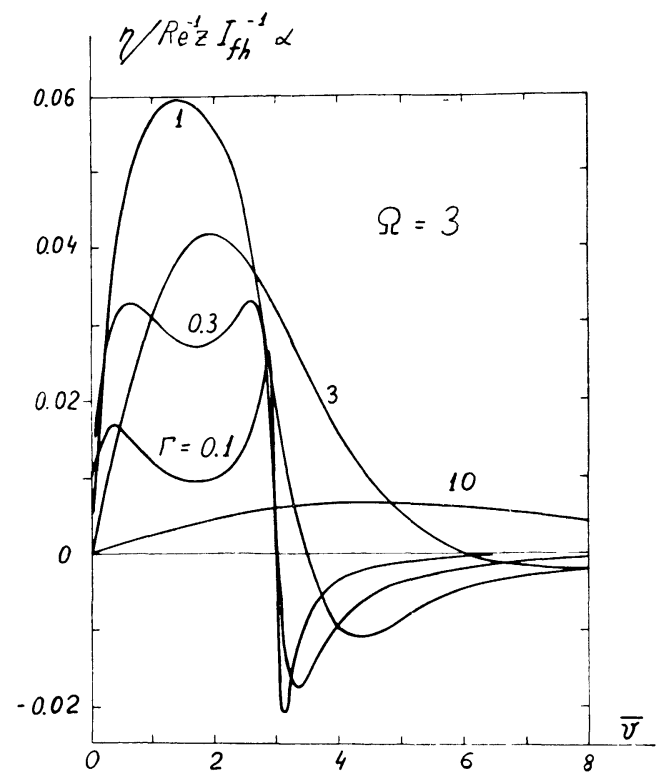

FIG. 7. - The variation of the junction response at the action of the fluctuations. At $\Gamma \ll 1$ the response corresponds to Kanter-Vernon formula (4.1) with the peculariarity at $\bar{v}=\Omega$ smoothed down using the expression (4.10).

Applying monochromatic signal $\left({ }^{17}\right)$ of a power $p=P / I_{0} V_{0}$ to hf circuit at mentioned conditions one obtains harmonic current through the junction of the amplitude

$$
\left(i^{(1)}\right)^{2}=\frac{8 p r_{\mathrm{c}}}{\left|z(\Omega)+z_{\mathrm{e}}\right|^{2}}
$$

and given above expressions for the response are valid. We obtain for dimensional volt-to-watt responsivity $\eta \equiv|\widetilde{\widetilde{V}}| / P$

$$
\begin{aligned}
& \eta=4 \alpha \Gamma(\operatorname{Re} z)^{-1} \cdot I_{\mathrm{fh}}^{-1}\left|\frac{\tilde{\bar{v}}}{\left(i^{(1)}\right)^{2}}\right|, \\
& \alpha=4 r_{\mathrm{c}} \operatorname{Re} z /\left|z+z_{\mathrm{e}}\right|^{2} .
\end{aligned}
$$

Comparing these results with the experiment one should keep in mind that incident hf signal is usually modulated by low frequency signal and output signal is effective voltage $V_{M}$ of the first harmonic of the variation of $\bar{V}$ with this frequency. Sometimes for

(17) Since such detector is quadratic its response to complicated signal is simple sum of its responses to independent frequency components of the signal. 
this case one determines a responsivity as $\eta_{\mathrm{M}}=\beta \eta$ where $\beta=\sqrt{2} / \pi$ for square-wave modulation for instance.

As it follows from (4.15) the highest responsivity at low noise is reachable in the inertial region $(\bar{v}<\Omega)$ and

$$
\eta=\alpha(\operatorname{Re} z)^{-1} I_{\mathrm{fh}}^{-1}\left(r_{\mathrm{d}} \Gamma / \bar{i} \Omega^{2}\right) .
$$

The similar expression was given in the work [61]. It gives values of $\eta$ five times better than experimental values for comparatively low resistance junctions (small $\Gamma$ ) [61] if to assume that $\alpha=1$. If to take into account unavoidable losses in the electrodes it is more reasonable to explain this discrepancy by low matching parameter $\alpha$ but not by the influence of the parasitic capacity of the junction assumed by authors of the work [61].

An example of the dependence $\eta(\bar{v})$ at different $\Gamma$ is shown at figure 7 .

We find the noise equivalent power $(N E P)$ equating signal voltage $\bar{V}=\eta \times N E P$ with mean-square-root value of noise voltage across the junction in the If output bandwidth $H_{\mathrm{M}}$ at frequencies much less than $\omega$ :

$$
<\bar{V}^{2}>=V_{0}^{2} s_{v}(0) \cdot\left(2 H_{\mathrm{M}} / \omega_{0}\right) \text {. }
$$

We have in dimensional units

$$
\begin{aligned}
N E P & =\frac{1}{\sqrt{2}} \alpha^{-1} \Gamma^{-1} \operatorname{Re} z \times \\
& \times s_{v}^{1 / 2}(0)\left|\frac{\tilde{\bar{v}}}{\left(i^{(1)}\right)^{2}}\right|^{-1} \cdot k T \cdot\left(\omega_{0} \cdot H_{\mathrm{M}}\right)^{1 / 2} .
\end{aligned}
$$

We obtain from (4.1) and (2.28) at $\bar{v} \ll \Omega$ for region of small fluctuations

$$
\begin{aligned}
N E P=2 \alpha^{-1} \operatorname{Re} & z\left[\left(2 \bar{i}^{2}+1\right) / \pi\right]^{1 / 2} \times \\
& \times \Gamma^{-1 / 2} \Omega^{2} k T\left(\omega_{0} H_{M}\right)^{1 / 2} .
\end{aligned}
$$

The choosing of optimal values of parameters is dependent essentially on operating conditions of the detector.

If the noise of lf amplifier for measuring the response of the junction is much larger than its intrinsic noise then main parameter of the detector is its responsivity $\eta$. It is seen from (4.15) that to increase $\eta$ it is necessary at first to increase the characteristic frequency $\omega_{0}$ of the junction decreasing $\Omega$ in the same time. However possibilities of increasing $\omega_{0}$ are always limited (see the part 1). Let $\Omega$ is fixed. Consider the dependence of $\eta$ on $\bar{v}$ and the junction resistance $\Gamma$ (1.15). The analysis of the expression (4.16) and figure 7 shows one can obtain maximum values of $\eta$ in the regions $\Gamma \widetilde{>} 1$ (at $\Omega \widetilde{>}$ ) or $\bar{v}<\Gamma^{1 / 3}$ (at $\Omega \ll 1$ ) i. e. where expression (4.16) is not valid and one is to find $\eta$ from (4.15) calculating $\tilde{\bar{v}}$ and $\operatorname{Re} z$ numerically using expressions (4.12) and (2.41). We have carried out such calculations separately for $\Omega \geqslant 1$ and $\Omega=0$ from which one can also find approximately the characteristics at $0<\Omega<1$.

The dependences of $\eta$ on $\Gamma$ at $\Omega=0$ are shown at figure 8. Optimal values of $\bar{v}$ are also calculated for each $\Gamma$ and shown at the same figure. Two curves (I and II) for each quantity correspond to different cases of matching the junction with hf external circuit. The curves I correspond to bad matching : $\left|z_{\mathrm{e}}\right| \gg|z| \simeq 1$ and matching factor

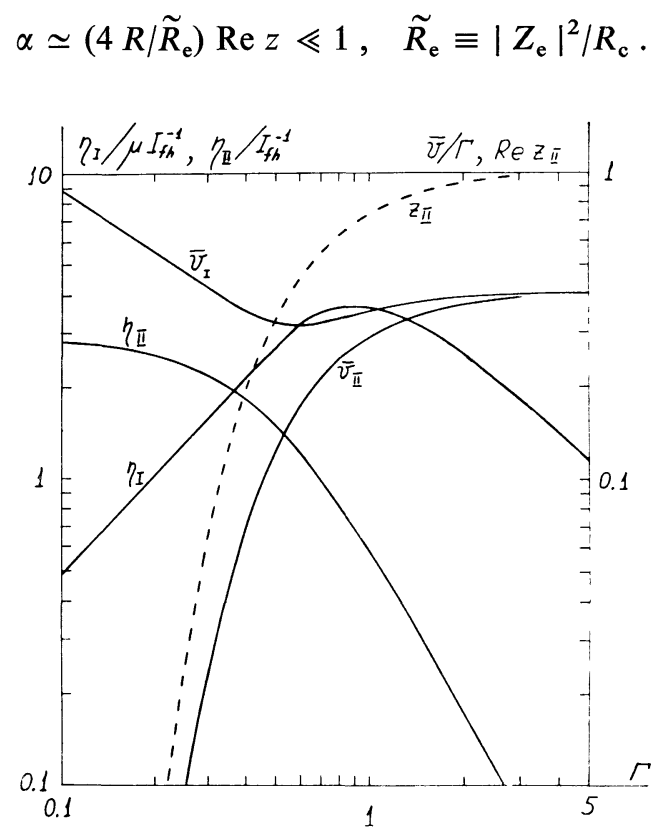

FIG. 8. - The dependences of the responsivity and also the bias voltage at which this responsivity is achieved on the junction resistance $R=\Gamma R_{\mathrm{f}}$ for frequencies $\omega \ll \omega_{0}$. The curves I and II correspond to different cases of the matching (see the text). The dashed line is the differential resistance $r_{\mathrm{d}}$ of the junction at the voltage $\bar{v}_{\mathrm{II}}$

The parameter $\mu$ is $\mu=R_{\mathrm{c}} R_{\mathrm{f}} / 2\left|Z_{\mathrm{e}}\right|^{2}$. The curves II correspond to optimal matching at each point :

$$
\alpha=1\left(z_{\mathrm{e}}=z^{*}, r_{\mathrm{c}}=\operatorname{Re} z\right) .
$$

It is seen that maximum value of $\eta$ at $\Omega=0$ in the case $\mathrm{I}$ is obtained at $\Gamma \simeq 1 \mathrm{i}$. e. for so high resistance junctions that their $i-v$ curve is smoothed down completely by fluctuations. At optimal matching (the case II) the value of responsivity increases infinitely at decreasing of the junction resistance $(\Gamma)$. However this value is being reached at superconducting region of the $i-v$ curve where the junction impedance becomes very low. The responsivity increases very slowly in this case and the value

$$
\eta_{\max } \simeq 3 / I_{\mathrm{fh}}
$$

can be taken as the absolute maximum of the responsivity of the wide-band detector. For thermal noise at $T=4.2 \mathrm{~K} \eta_{\max } \simeq 8.5 \times 10^{6} \mathrm{~V} / \mathrm{W}$. This is twenty times better than experimental value achieved to this moment [79].

The dependence of $\eta_{\max }$ on $\Omega$ for $\Omega \geqslant 1$ is shown 
at figure 9. The curves corresponding only to case II (the junction is matched with the circuit) are shown at this figure. The optimal value of $\eta$ at such frequencies has being reached at so large values of $\Gamma$ that the $i-v$ curve is smoothed down strongly and owing to this optimal impedance of external circuit is practically equal to the normal resistance of the junction. We have for instance for two-millimeter waveband region $\eta_{\max } \simeq 1.2 \times 10^{6} \mathrm{~V} / \mathrm{W}$ at $V_{0}=300 \mu \mathrm{V}(\Omega=1), T=4.2 \mathrm{~K}$ and $R_{\mathrm{opt}} \simeq 10^{3} \mathrm{ohms}$.

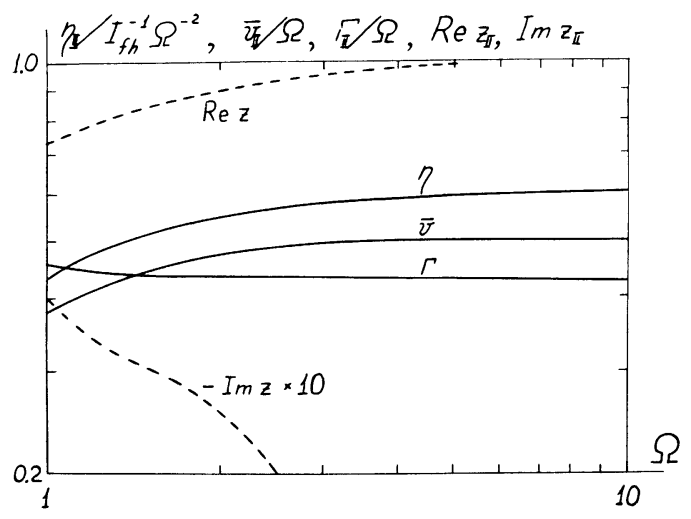

FIG. 9. - The maximum responsivity $\eta$ and corresponding values of $v$ and $\Gamma$ at $\Omega \geqslant 1$. The dashed lines are the components of the junction impedance.

In the case when the noise of the detector consists predominantly of the intrinsic noise of the junction then main parameter of the detector is the NEP. The dependences of values of the NEP at $\Omega=0$ optimized with respect to $\bar{v}$ are shown at figure 10 . At bad matching (the case I) the optimal value of the junction resistance exists $(\Gamma \simeq 1)$ at which the $N E P$ reaches the minimum. At optimal matching (the

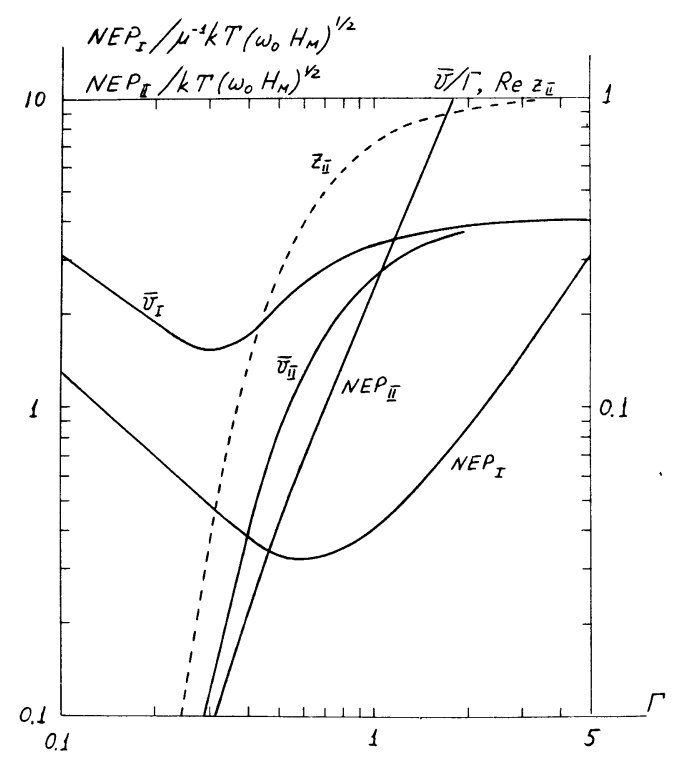

FIG. 10. - The minimum values of the NEP of the detector and the bias voltages at which they are achieved for low frequencies $\left(\omega \ll \omega_{0}\right)$. The dashed line is $r_{\mathrm{d}}$ at $\bar{v}=\bar{v}_{\mathrm{II}}$. case II) the NEP decreases sharply at decreasing of $\Gamma$. But small values of $\Gamma$ correspond to superconducting region of the $i-v$ curve and the value of impedance of the external circuit required for the matching $\left(z_{\mathrm{e}} \simeq z^{*}\right)$ decreases sharply. At $\Omega \geqslant 1$ the difference between two cases of the matching becomes smaller. The dependences of the NEP on $\Gamma$ at $\Omega \geqslant 1$ are shown at figure 11 for bad matching (the case I) and at figure 12 for optimal matching (the case II). It is seen that at optimal matching for $\Omega \sim 1$

$$
(N E P)_{\min } \simeq 4 \Omega^{2} k T\left(\omega_{0} \quad H_{\mathrm{M}}\right)^{1 / 2}
$$
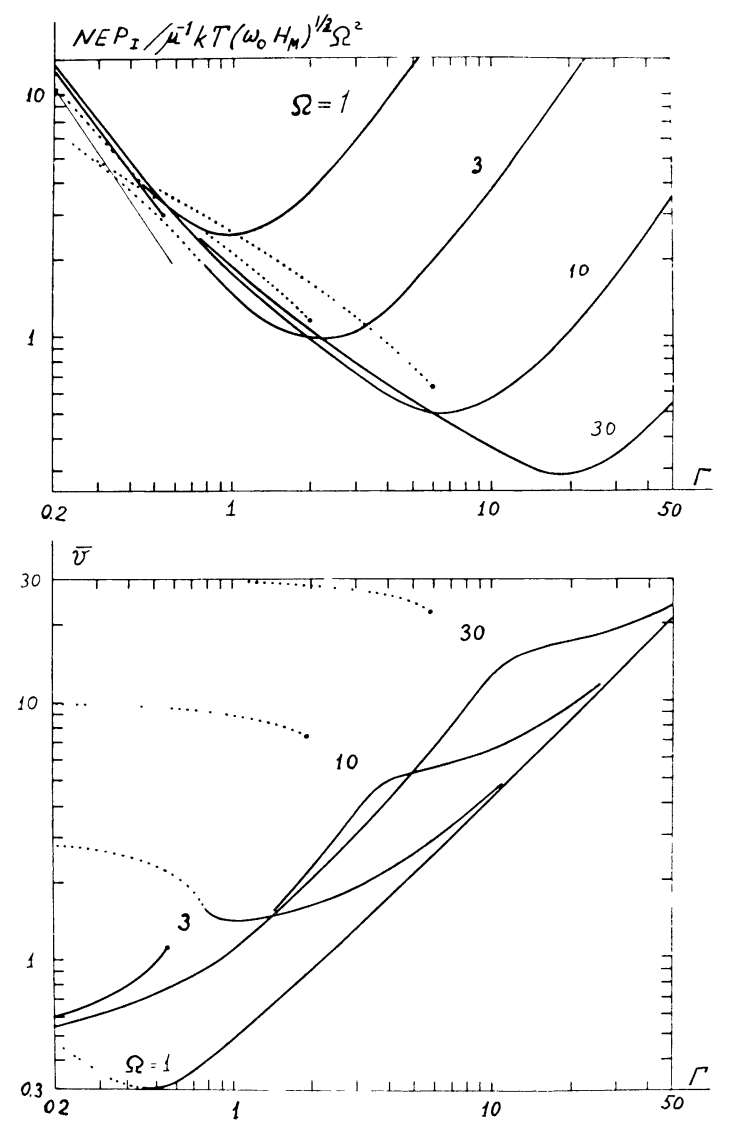

Fig. 11. - The minimum values of the $N E P$ at $\Omega \geqslant 1$ corresponding to bad matching.

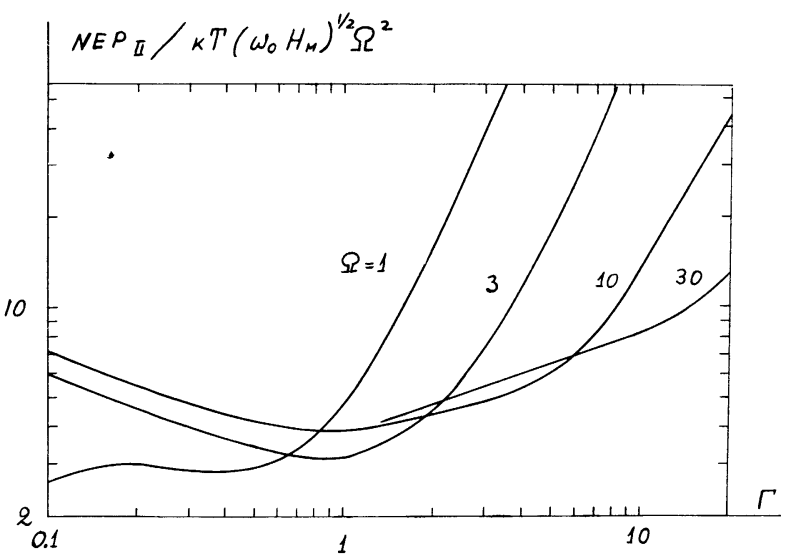

Fig. 12. - The minimum values of the $N E P$ at $\Omega \geqslant 1$ corresponding to optimal matching. 
and these minimum values correspond to the values of $\Gamma$ a little less than ones corresponding to maximum values of $\eta$. It is connected with the increasing of fluctuations of voltage at the increasing of the junction resistance. The values determined by (4.19) and (4.20) are approximately an order and half better than obtained experimentally to this moment.

As it follows from the expression (4.15) and figure 9 the frequency dependence of the detector responsivity in wide-band regime is close to the dependence $\eta \sim \alpha(\omega) . \omega^{-2}$ at $\omega>\omega_{0}$ that corresponds well to experimental data [61], [70], [75].

4.3 THE SELECTIVE REGIMES OF THE DETECTORS. The selective regimes $(\bar{v} \simeq \Omega)$ have that peculiarity that strong anomalies of junction impedance are possible (see the part 3) which practically do not have an influence as we have seen in case of wideband regime. Besides the combination frequency $(2 \bar{v}-\Omega)$ voltage of essential value can appear across the junction at $\bar{v} \simeq \Omega$ as it was shown during the analysis of the down-converters. This peculiarity does not allow in common case to use the presented above expressions obtained to the assumption that the current flowing through the junction is harmonic. This is the reason why the results of the analysis of such regime carried out in the works [77], [78] are valid only in some special cases (for instance in the case of bad matching the junction with the hf circuit).

On the other hand the optimal values of parameters in selective regimes can be reached at small noise i. e. at comparatively small resistance of the junction $\left(\Gamma_{1} \ll \Omega\right)$. This is seen for instance from figures 8 and 12. Therefore it turned out that all characteristics of the detector can be found analytically.

Consider two regimes of detector operation at $\bar{v} \simeq \Omega$ similar to corresponding regimes of the downconverter.

1) If the bandwidth of the external circuit is sufficiently wide (3.22) then to find a response to $\mathrm{hf}$ signal one can use the matrix (3.23) crossing out third row and third column from it because of the absence of the circuit at frequency $|\Delta|$. We obtain directly from this matrix the following result. If the emf corresponding to incident power $P$ is

$$
e \cos \Omega t\left(\Omega=\bar{v}+\Delta, e^{2} / 8 r_{\mathrm{c}}=p=P / I_{0} V_{0}\right)
$$

then hf current $(\Omega \approx 1)$

$$
\begin{aligned}
& i_{e}= \frac{e}{\left(1+r_{\mathrm{h}}\right)^{2}}\left\{\left[1+r_{\mathrm{h}}+\frac{1}{2(\bar{i}+\bar{v}) \Delta}\right] \times\right. \\
&\left.\quad \times \cos (\bar{v}+\Delta) t+\frac{1}{2(\bar{i}+\bar{v}) \Delta} \cos (\bar{v}-\Delta) t\right\} \\
&=\frac{e}{1+r_{\mathrm{h}}} \cos (\bar{v}+\Delta) t+ \\
& \quad+\frac{e}{(\bar{i}+\bar{v}) \Delta\left(1+r_{\mathrm{h}}\right)^{2}} \cos \bar{v} t \cos \Delta t . \quad(4.21)
\end{aligned}
$$

Since the amplitude of the current component (of frequency $\Omega=\bar{v}+\Delta$ ) producing the response does not depend on $\Delta$ then the expression (4.21) can be used for $\Gamma \neq 0$ also. We obtain directly from (4.10) and (4.21) the response

$$
<\tilde{\bar{v}}>=\frac{e^{2} \Delta}{8 \Omega^{2}\left(\Delta^{2}+\Gamma_{1}^{2}\right)\left(r_{\mathrm{h}}+1\right)^{2}} .
$$

Thus the effective impedance of the junction is equal to the unity $(R)$ i. e. the anomalous impedance does not reveal itself in relatively wide-band external circuit. Taking into account the expression for signal power $P=I_{0} V_{0} e^{2} / 8 r_{\mathrm{c}}$ we have at small losses of hf circuit $\left(r_{\mathrm{c}}=r_{\mathrm{h}}\right)$

$$
\eta=\alpha I_{\mathrm{fh}}^{-1} \Omega^{-2} \frac{|\Gamma \Delta|}{2\left(\Delta^{2}+\Gamma_{1}^{2}\right)}
$$

and coupling factor $\alpha=4 r_{h} /\left(r_{h}+1\right)^{2}$ does not depend on the choice of operating point at the $i-v$ curve. At $r_{\mathbf{h}} \ll 1(\alpha \gg 1)$ the expression (4.23) for the responsivity is trivial consequence of the expression (4.10). It is seen from (4.23) that $\eta$ is the oddresonant function of $\Delta \mathrm{i}$. e. of signal frequency and the position of the center of this resonance is determined by choosing of the bias voltage. Therefore this regime can be called a regime of " self-selection ". The experimental investigations of this regime are described in the works [61], [72]-[74].

Taking into account $(2.23)$ we obtain for the $N E P$

$$
\begin{aligned}
N E P= & 2 \sqrt{\frac{2}{\pi}} I_{\mathrm{fh}} V_{0} \Omega \times \\
& \times \frac{\Gamma_{1}^{2}+\Delta^{2}}{|\Delta| \Gamma}\left(\bar{i}^{2}+\frac{1}{2}\right)^{1 / 2}\left(\Gamma H_{\mathrm{M}} / \omega_{0}\right)^{1 / 2}
\end{aligned}
$$

The expressions (4.23) and (4.24) show that optimal values of $\eta$ and $N E P$ are reaching at $\Delta= \pm \Gamma_{1}$, $r_{\mathrm{h}}=1$

$$
\begin{aligned}
\eta_{\max } & =\frac{1}{4 I_{\mathrm{fh}}} \cdot \frac{1}{\Omega^{2}} \\
(N E P)_{\min } & =\frac{8}{\sqrt{\pi}} \Omega^{2} k T\left(H_{\mathrm{h}} H_{\mathrm{M}}\right)^{1 / 2}
\end{aligned}
$$

where the quantity $H_{\mathrm{h}}=2 \Gamma_{1} \omega_{0}$ can be taken as hf bandwidth of the detector.

It is necessary to increase $\omega_{0}$ (i. e. to decrease $\Omega$ ) for the improvement of the characteristics of the detector as in wide-band case. It is seen from (4.25) that maximum responsivity does not depend on the junction resistance (while $\Gamma_{1} \ll \Omega$ ). The comparison of the expression (4.25) with figure 10 shows that this responsivity at $\Omega \widetilde{>} 1$ is somewhat less than in wide-band regime.

2) If the bandwidth of the hf circuit (cavity) is narrow (3.31) that corresponds to the experimental 
works [43], [71] then as during the analysis of linear effects the current $i_{\mathrm{e}}$ can be assumed as harmonic at frequency $\Omega=\bar{v}+\Delta=\Omega_{\mathrm{h}}+\Delta_{\mathrm{h}}$, where $\Omega_{\mathrm{h}}$ is cavity own frequency, $\left|\Delta_{\mathrm{h}}\right| \ll \Delta$. Using the expression (3.12) we obtain for current amplitude

$$
\begin{gathered}
i^{(1)}=\frac{e}{\tilde{r}}\left[1+\frac{\tilde{\Delta}_{\mathrm{h}}^{2}}{\tilde{h}_{\mathrm{h}}^{2}}\right]^{-1}, \\
\tilde{r}=1+r_{\mathrm{h}}-\frac{1}{2(\bar{i}+\bar{v})} \cdot \frac{\Delta}{\Delta^{2}+\Gamma_{1}^{2}}, \frac{\tilde{h}_{\mathrm{h}}}{h_{\mathrm{h}}}=\frac{\tilde{r}}{r_{\mathrm{h}}}
\end{gathered}
$$

where $\tilde{\Delta}_{\mathrm{h}}$ and $\tilde{h}_{\mathrm{h}}$ are frequency shift from resonance and half of the bandwidth of the cavity at taking into account the junction impedance. Using now the expression (4.10) we obtain that the responsivity at the resonance frequency $\left(\tilde{\Delta}_{\mathrm{h}}=0\right)$

$$
\eta=\frac{4}{r_{\mathrm{h}}} I_{\mathrm{fh}}^{-1} \Omega^{-2} \frac{\Gamma|\Delta|}{2\left(\Delta^{2}+\Gamma_{1}^{2}\right)} \cdot\left(\frac{h_{\mathrm{h}}}{\tilde{h}_{\mathrm{h}}}\right)^{2} .
$$

It is seen that main peculiarity of such detector is the possibility of unlimited increasing of the responsivity owing to regeneration of cavity connected with negative (at $\Delta>0$ ) resistance of the junction. In other words at $\tilde{\tau} \rightarrow 0 \tilde{h}_{\mathrm{h}} \rightarrow 0$ and $\eta \rightarrow \infty$. In fact the circuit in this case is the combination of regenerative amplifier (see part 3) and the detector. The described effects, in particular the narrowing of the bandwidth $\tilde{h}_{\mathrm{h}}$, were observed in the work [71].

At low regeneration $\left(\tilde{h}_{\mathrm{h}} \simeq h_{\mathrm{h}}\right)$ the regime of operation using narrow-band cavity differs from the regime of self-selection only by the fact that the portion with bandwidth $2 h_{\mathrm{h}} \omega_{0}$ is cut from the spectrum of input signal i. e. external frequency selection takes place. Therefore we will consider further only the most interesting case of strong regeneration

$$
\left.\tilde{r} / r_{\mathrm{h}}=\tilde{h}_{\mathrm{h}} / h_{\mathrm{h}} \ll 1\right) \text {. }
$$

And as it follows from (4.27) and (4.28) the product $\eta\left(2 \tilde{h}_{\mathrm{h}} / \Omega\right)^{2}$ is fixed. Here $2 \tilde{h}_{\mathrm{h}} / \Omega$ is relative bandwidth of the regenerated circuit. The maximum value of this « detection efficiency »

$$
\left[\eta\left(\frac{2 \tilde{h}_{\mathrm{h}}}{\Omega}\right)^{2}\right]_{\max }=\frac{Q-1}{\rho_{\mathrm{h}}^{2} I_{\mathrm{fh}}} \Omega^{-2}
$$

corresponds to $\Delta=\Gamma_{1}, r_{\mathrm{h}}=Q-1$. Comparing this value with corresponding value in the regime of self-selection one can see that this value is larger in the latter case at $Q>1, \rho_{\mathrm{h}}^{-2}$.

For the NEP at strong regeneration when the noise of the detector is determined by the noise of the cavity we obtain from (3.34)

$$
\begin{aligned}
N E P=\frac{\Omega^{2}+\frac{3}{2}}{\Omega}+\left(\Omega^{2}+1\right)^{1 / 2} \times \\
\times\left\{\frac{\Delta}{2}-\left(\Delta^{2}+\Gamma_{1}^{2}\right)\left[\Omega+\left(1+\Omega^{2}\right)^{1 / 2}\right]\right\}^{-1} \\
\times k T\left(\tilde{H}_{\mathrm{h}} H_{\mathrm{M}}\right)^{1 / 2}
\end{aligned}
$$

where the dimensional hf bandwidth of the detector $\widetilde{H}_{\mathrm{h}}=\tilde{h}_{\mathrm{h}} \omega_{0}$ is calculating now taking into account the regeneration. At the tuning the detector to the maximum "detection efficiency » (4.29) we have

$$
N E P=8 k T\left(\Omega^{2}+\frac{3}{2}\right)\left(Q^{-1}-Q^{-2}\right)^{-1}\left(\tilde{H}_{\mathrm{h}} H_{\mathrm{M}}\right)^{1 / 2}
$$

and at $\Omega \approx 1$ one can to find minimum value of the $N E P$

$(N E P)_{\min }=16 k T \Omega^{2}\left(1-Q^{-2}\right)^{-1} \cdot\left(\tilde{H}_{\mathrm{h}} H_{\mathrm{M}}\right)^{1 / 2}$

at $\Delta=1 / 8 \Omega, r_{\mathrm{h}}=1$. The comparison of expressions (4.32) and (4.26) for two regimes of operation of selective detector shows that the gain at the regenerative regime (the external selection) can be obtained only owing to narrowing of the effective hf bandwidth of the detector.

4.4 THE SELF-DETECTION. - The current $i_{\mathrm{e}}$ produces a variation of the $i-v$ curve of the junction also in that case when it is caused by not external signal but by the voltage of own Josephson oscillations. If the impedance of external circuit is sufficiently large then considering problem can be solved using the perturbation theory. As it is shown in the works [80], [81] the variation of the $i-v$ curve in the absence of fluctuations is equal in this case

$\tilde{\bar{i}}=-\frac{\bar{v}}{\bar{i}(\bar{i}+\bar{v})} r_{\mathrm{e}}^{-1}(\bar{v}), r_{\mathrm{e}}^{-1}(\bar{v})=\operatorname{Re}\left[z_{\mathrm{e}}^{-1}(\bar{v})\right]$

i. e. is proportional to the real part of the admittance at the frequency of the first harmonic of the Josephson oscillations.

Unlike to the Josephson structures with large capacitance (tunnel structures [1]) the connecting the external circuit to the junction produces " lowering of the $i-v$ curve " $i$. e. decreasing of current $i$ at given voltage $\bar{v}$. These effects were observed many times experimentally [41], [82]-[84]. More detailed consideration of them will be given in the part 5 .

5. The properties of the junction at the action of large external signal. - 5.1 THE EXTERNAL ACTION ON THE JUNCTION. - We will study in this part the behaviour of the junction at large coupling current $i_{\mathrm{e}}$ when the amplitude of at least one of its frequency components is of order or larger than unity. Unlike the case of small values $i_{\mathrm{e}}$ the strict analytic solution of basic eq. (1.12) is absent in this case even at $i_{\mathrm{f}}=0$ and therefore we are to be satisfied with approximate analytical or numerical methods.

One of the most useful cases when the eq. (1.12) can be solved analytically is the case when $\Omega \gg 1$ and as it was mentioned above the obtained results actually are valid with good accuracy at $\Omega \sim 1$. Therefore we will find in this part analytical solutions of problems for the case $\Omega \gg 1$ supplementing them with results of numerical calculations for $\Omega<1$. 
Let the external circuit has sufficiently high impedance $\left(\left|z_{\mathrm{e}}\right| \gg 1\right)$. Then the component of the current $i_{\mathrm{e}}$ caused by the Josephson oscillations of the junction is small. At the action on the circuit of the monochromatic external signal of the power $P$ the current $i_{\mathrm{e}}$ is also harmonic and $i_{\mathrm{e}}=i^{(1)} \sin \Omega t, i^{(1)} \simeq e /\left|z_{\mathrm{e}}\right|$, $P=I_{0} \quad V_{0} e^{2} / 8 \quad r_{\mathrm{c}}$. When fluctuations are absent $\left(i_{\mathrm{f}}=0\right)$ and the operating point of the junction is at the $n$th step $\left({ }^{18}\right)$ of the $i$ - $v$ curve then the solution of the eq. (1.12) in zero-order approximation with respect to $\Omega^{-1}$ is $\varphi^{(0)}=\bar{v} t+\chi, \bar{v}=n \Omega$, and first correction to it is

$\varphi^{(1)}=-\left(i^{(1)} / \Omega\right) \cos \Omega t+(1 / n \Omega) \sin (n \Omega t+\chi)$.

Hence we have

$\bar{i}=\overline{\dot{\varphi}}-\overline{\sin \varphi} \simeq \bar{v}+\frac{1}{2 \bar{v}}+J_{n}\left(\frac{i^{(1)}}{\Omega}\right) \sin (\chi-n \pi / 2)$.

The first two terms in the right-hand side of (5.1) correspond to autonomous $i-v$ characteristic

$$
\bar{i}=\left(\bar{v}^{2}+1\right)^{1 / 2} \text { at } \bar{v} \gg 1
$$

and the last term is the variation (the step) of the $i-v$ curve under influence of the external signal. The well-known result follows directly from (5.1) that the value of $n$th step at $\Omega \gg 1$ is the same as for Josephson structures with large capacitance

$$
\tilde{i_{n}}=2 J_{n}\left(\frac{i^{(1)}}{\Omega}\right)
$$

$\tilde{\bar{i}}_{n}$ at $n=0$ is doubled value of the critical current.

The dependences $\tilde{\tilde{i}_{n}}\left(i^{(1)}\right)$ at $\Omega \approx 1$ are different from (5.2). The calculations of them have been carried out in the works [87]-[90]. At $i^{(1)} \sim 1$ for $\Omega \rightarrow 0$ the values of first $N$ steps (where $N \simeq \Omega^{-1}$ ) are equal and the maximum magnitudes of them are

$$
\left(\tilde{\overline{i_{n}}}\right)_{\max } \simeq 2 \Omega \text {. }
$$

The comparison of theoretical and experimental dependences $\tilde{\overline{i_{n}}}\left(i^{(1)}\right)$ is one of the best methods of verification of adequacy of given junction to the resistive model and also of obtaining the parameter $\Omega$ and hence $\omega_{0}$.

From our point of view the graphs given at figure 13 [48], [89] are the most convenient for this purpose. The values $\kappa_{m}$ are

$$
\kappa_{m}=\left(i_{2 m}^{(1)}-i_{1 m}^{(1)}\right) / i_{1 m}^{(1)}
$$

where $i_{k m}^{(1)}$ is the value of the external current amplitude at which $m$ th step becomes zero for the $k$ th time.

(18) The effect of the appearance of Josephson steps at the $i$-v-characteristics is well-known [1] and we will not describe it here. They were observed many many times at voltages up to $15 \mathrm{mV}(n=103)$ [85] and at frequencies up to $2.5 \mathrm{THz}$ [86].

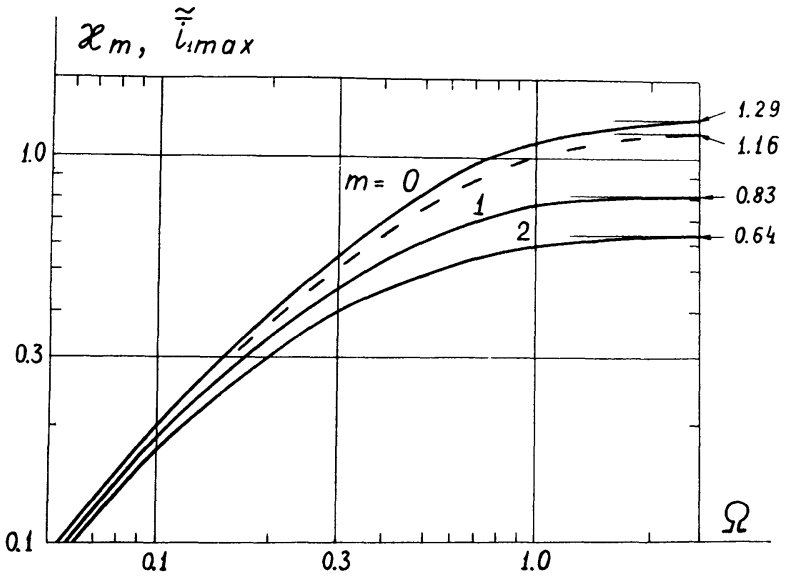

FIG. 13. - The dependence of the values of $\kappa_{m}$ (solid lines) and the value of $\left.\widetilde{\overline{i_{1}}}\right)_{\max }$ (dashed line) on the frequency of the external action. The arrows show the corresponding values at $\Omega \gg 1$ obtained from (5.2).

The dependence $\left(\tilde{\overline{i_{1}}}\right)_{\max }$ on $\Omega$ is also shown at figure 13 by dashed line. But the using this curve is impeded with the smoothing down of the edges of the steps by fluctuations. Small fluctuations do not influence on values $\kappa_{m}$.

Note another two interesting circumstances.

Firstly subharmonic steps $i$. e. steps at voltages $\bar{v}=(n / m) \Omega(m \neq 1)$ are absent for the resistive model [87]-[90]. It is connected not with the absence of harmonics of the Josephson oscillations (as it is seen for instance from (2.5) their spectrum is very reach with harmonics at $\Omega \approx 1$ ) but with the specific relations of amplitudes and phases of these harmonics. Either even small deviation of the dependence $I_{\mathrm{s}}(\varphi)$ from harmonic law or the connection of not large capacitance in parallel with the junction causes the appearance of these steps [91].

Secondly the values of steps in the resistive model oscillate passing though zero during variation of $i^{(1)}$ at all values of $\Omega$. If the dependence $I_{\mathrm{s}}(\varphi)$ in real junction is different from harmonic one even a little,

$$
I_{\mathrm{s}}=I_{0}\left(\sin \varphi+\sum_{m=2}^{\infty} \varepsilon_{m} \sin m \varphi\right), \quad \sum_{m=2}^{\infty}\left|\varepsilon_{m}\right| \ll 1,
$$

then passings through zero will not take place. Really we have from (5.5) at $\Omega \gg 1$

$$
\left(\tilde{\overline{i_{0}}}\right)_{\min }=\max _{x}\left|\sum_{m=2}^{\infty} a_{m} \varepsilon_{m} \sin m x\right|
$$

where $a_{m}=J_{0}\left(m \cdot j_{0,1}\right), j_{0,1}$ is the first root of the equation $J_{0}(x)=0$. If at least one of factors $\varepsilon_{m} \neq 0$ then $\left(\tilde{\overline{i_{0}}}\right)_{\min }<0$.

The analysis of the experimental results for the point contacts and the bridges of small dimensions shows that the value $\left.\tilde{\widetilde{i_{0}}}\right)_{\min }$ is close to zero in very most cases of such structures. Therefore one can 
assert that the dependence $I_{\mathrm{s}}(\varphi)$ is pretty close to harmonic one for the most of such structures and observed discrepancies with the resistive model are caused by another effects (perhaps by nonlinearity and dispersion of the normal component of the current [1]).

5.2 THE JosephSON RADIATION TO THE EXTERnAL CIRCUIT. - Let now an external signal is absent and the impedance of the external circuit is comparable with the normal resistance of the junction $\left(\left|z_{\mathrm{e}}\right| \approx 1\right)$. In this case the voltage of the Josephson oscillations across the junction will result in the arising of the current $i_{\mathrm{e}}$ of considerable magnitude.

This problem can be solved analytically [90], [92] for instance in the case when the impedance $z_{\mathrm{e}}$ is active and independent on frequency for all frequencies except $\omega=0: z_{\mathrm{e}}(\Omega)=r_{\mathrm{e}}=$ Cte. Physically almost similar conditions take place at the insertion of the junction into a long line without reflections. Carrying out not complicated transformations we obtain

$\bar{i}=\left[1+\left(1+r_{\mathrm{e}}^{-1}\right)^{2} \bar{v}^{2}\right]^{1 / 2}-r_{\mathrm{e}}^{-1} \bar{v} \quad(\bar{v}>0)$,

which is in agreement with (4.33) at $r_{\mathrm{e}}^{-1} \ll 1$.

As it is seen from the expression (5.7) the $i-v$ characteristic caves in down (see figure 1 in the work [90]) owing to the self-detection and becomes of " angle " shape in the limit case $\left(r_{\mathrm{e}} \rightarrow 0\right)$

$$
\bar{v}= \begin{cases}0 & \bar{i}<1, \\ \bar{i} & \bar{v}>0 .\end{cases}
$$

Such « angle » is the limit for the $i$ - $v$ curve at decreasing of $z_{\mathrm{e}}$ for any type of the load.

We obtain for the power of the radiation at the fundamental harmonic

$$
P_{1}=\frac{\bar{V}^{2}}{R}\left\{\left[1+\left(1+r_{\mathrm{e}}^{-1}\right)^{2} \bar{v}^{2}\right]^{1 / 2}-\left(1+r_{\mathrm{e}}^{-1}\right) \bar{v}\right\} \text {. }
$$

The dependences of the maximum value of $P_{1}$ with respect to $r_{\mathrm{e}}$ and also this optimal value of $r_{\mathrm{e}}$ on $\Omega=\bar{v}$ are shown at figure 14 by dashed lines. It is seen from figure 14 that it is advantageous to increase $V_{0}$ at fixed frequency $\Omega$ untill we obtain $\Omega \approx 1$ and to make line impedance equal to

$$
\left(r_{\mathrm{e}}\right)_{\mathrm{o}_{\vec{r}} \mathrm{t}}=2 \sqrt{3} \Omega \approx 1 .
$$

Then the maximum power

$$
\left(P_{1}\right)_{\max }=I_{0} V_{\omega} / 3 \sqrt{3}
$$

is proportional to the critical current. So in the $\mathrm{X}$-band region $V_{\omega} \simeq 2 \times 10^{-5} \mathrm{~V}$ and for real junction with $I_{0}=3 \mathrm{~mA}\left(P_{1}\right)_{\max } \simeq 10^{-8} \mathrm{~W}$. To obtain such value of the power the long line having very small impedance $\left(r_{\mathrm{e}} \simeq 10^{-2} \mathrm{ohm}\right)$ is required. Experimental results are approaching nearer and nearer this value of the power [93].

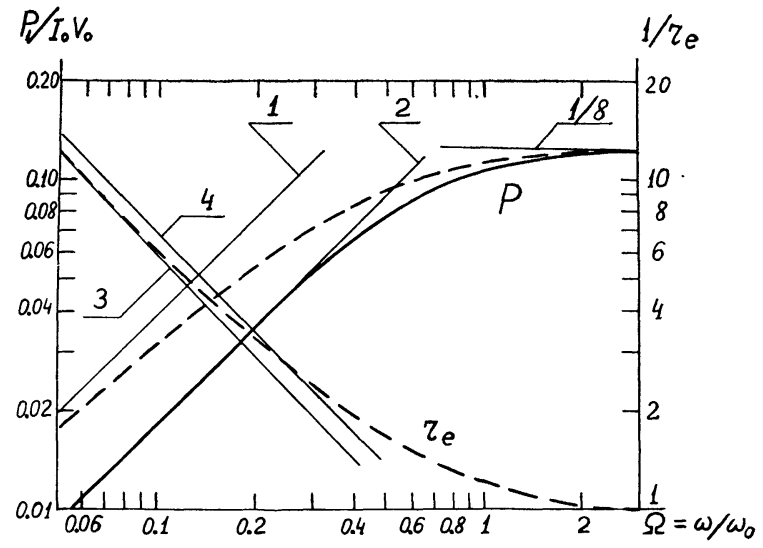

FIG. 14. - The dependence of maximum power $P_{1}$ of the radiation from the junction to the external circuit and corresponding value of the load resistance $\left(r_{\mathrm{e}}\right)$ on the radiation fre quency. The solid lines are for the cavity, the dashed lines are for the long line. The fine lines are asymptotes : (1) : $(2 \sqrt{3} / 9) \Omega$,

(2) : $[(\pi-2) / 2 \pi] \Omega,(3): 1 /(\sqrt{3} \Omega),(4):[\pi / 4(\pi-2)] / 2 \Omega$.

It is more convenient to match the junction with the resonator (cavity) of seires type which has at resonance frequency $r_{\mathrm{h}}=\rho_{\mathrm{h}} / Q_{\mathrm{h}}$, where $\rho_{\mathrm{h}}$ and $Q_{\mathrm{h}}$ are wave impedance and quality respectively of the resonator. When the resonator has high quality $\left(Q_{\mathrm{h}} \gg 1\right)$ and fluctuations are absent then the current $i_{\mathrm{e}}$ can be assumed to be harmonic and the problem is identical to the external action problem. It was solved in the work [80].

The self-detection produces the arising of narrow wells at the $i-v$ curve with relative bandwidth $\sim Q_{\mathrm{h}}^{-1}$ (see Fig. 1 in the work [80]). The same dependences as for the long line are shown by solid lines at figure 14 for the resonator at precise tuning of it (only asymptotes $\left({ }^{19}\right)$ are shown for $\left.\left(r_{\mathrm{e}}\right)_{\mathrm{opt}}\right)$. It is seen that the maximum power of the radiation to the resonator at $\Omega \approx 1$ is somewhat less than to the long line. It is connected with the fact that the resonator in contrast to the long line does not shunt highest harmonics of the oscillations in the junction, the presence of which results in the suppressing action on oscillation process. This difference however is not large. Therefore given above estimation for the maximum power which can be obtained from real junction is valid also in case of insertion of the junction to the resonator where it is not essentially difficult to realize required values of $r_{\mathrm{e}}\left(r_{\mathrm{e}} \ll 1\right)$.

There is an interesting case when the junction is inserted to the special line of finite length with $\left|z_{\mathrm{e}}\right| \simeq 1$ [83], [84]. Resonances of both series $\left(\left|z_{\mathrm{e}}\right|\right.$ decreases $)$ and parallel $\left(\left|z_{\mathrm{e}}\right|\right.$ increases $)$ types can be realized in such line (in practice in strip reso-

(19) It was found that depths of the wells at $r_{\mathrm{e}}=\left(r_{\mathrm{e}}\right)_{\text {opt }}$ for all values of $\Omega$ are within values

$$
\widetilde{\widetilde{i}}=-(0.45 \div 0.55) \times\left(i^{(0)}-\bar{v}\right)
$$

that can be used as a criterion of the matching. 
nator). They are located in pairs at the frequency axis in case of simple geometry. In this case peaks located in pairs at the $i-v$ curve reveal themselves on the background of common lowering of the $i$ - $v$ curve [83]. These peaks are directed down for the resonances of series type and up for the resonances of parallel type inherent in the tunnel Josephson structures [1].

5.3 The equations For High FReQuencies. To study in more detail processes taking place in the junction at $\bar{v} \simeq \Omega$ ( $\Omega$ is the frequency of the coupling current $i_{\mathrm{e}}$ ) we obtain auxiliary equations for case $\Omega \sim 1$. The deriving them is like the deriving KanterVernon formula (see the part 4) but the expansion is carrying out with respect to parameters $\Omega^{-1} \approx 1$, $i_{\mathrm{e}} \approx 1$. We are finding the solution of the basic eq. (1.12) in the form $\varphi=\varphi^{(0)}+\varphi^{(1)}$, where

$$
\begin{aligned}
\dot{\varphi}^{(0)} & =\bar{i}+i_{\mathrm{f}}-\tilde{\bar{i}}, \\
\dot{\varphi}^{(1)}+\cos \varphi^{(0)} \cdot \varphi^{(1)} & =i_{\mathrm{h}}-\sin \varphi^{(0)}+\tilde{\bar{i}} .
\end{aligned}
$$

We have used the fact that the spectra of the Josephson oscillations and the external signal at $\bar{v} \simeq \Omega$ and low noise $(\Gamma \ll \Omega)$ can be separated into two parts. One is lf current (frequencies $\ll \Omega$ ) and another is hf currents with relatively narrow bandwidth. The operating components of fluctuation current at $\Gamma \ll \Omega$ are also if ones. Slow time function $\tilde{\bar{i}}$ (its relative rate is much less than $\Omega$ ) should be chosen again from the requirement $\left\langle\overline{\dot{\varphi}}^{(1)}\right\rangle=0$. We obtain from (5.12) and (5.13) easily

$$
\begin{aligned}
\varphi^{(1)}=\left(1-\frac{\sin \varphi^{(0)}}{\bar{i}}\right) & \int^{t} \mathrm{~d} t^{\prime}\left(1+\frac{\sin \varphi^{(0) \prime}}{i}\right) \times \\
& \times\left(i_{\mathrm{h}}^{\prime}-\sin \varphi^{(0) \prime}+\tilde{\tilde{i}^{\prime}}\right)
\end{aligned}
$$

where dashes (') mean that given time functions are of argument $t^{\prime}$. Since the spectrum of $i_{\mathrm{h}}$ is narrow we can represent it in the form

$$
i_{\mathrm{h}}=i_{\mathrm{c}} \cos \varphi^{(0)}+i_{\mathrm{s}} \sin \varphi^{(0)}
$$

where $i_{\mathrm{c}, \mathrm{s}}$ are slowly varying amplitudes. Calculating $\varphi^{(1)}$ we obtain $\tilde{\bar{i}}$

$$
\tilde{\bar{i}}=\left(1-i_{\mathrm{s}}\right) / 2 \bar{i}
$$

and the voltage across the junction $v=\dot{\varphi}^{(0)}+\dot{\varphi}^{(1)}$. It is convenient to separate this voltage into If $\left(v_{1}\right)$ and $\mathrm{hf}\left(v_{\mathrm{h}}\right)$ components

$$
\begin{gathered}
v_{1}=\bar{v}+\dot{\chi}^{(0)}, \quad v_{\mathrm{h}}=i_{\mathrm{h}}-\sin \varphi^{(0)}, \\
\varphi^{(0)}=\bar{v} t+\chi^{(0)}
\end{gathered}
$$

where slowly varying phase $\chi^{(0)}$ satisfies an equation

$$
\dot{\chi}^{(0)}=i_{\mathrm{f}}+i_{1}+i_{\mathrm{s}} / 2 \bar{i} .
$$

The quantity $\bar{v}$ is mean voltage across autonomous function $\bar{v}=\sqrt{\bar{i}^{2}-1}$.
The set of eq. (5.15), (5.17), (5.18) is complete one, i. e. it gives a possibility to find components of the voltage across the junction at given currents $i_{\mathrm{h}}$ and $i_{1}\left({ }^{20}\right)$. We illustrate its application by two examples.

We have for autonomous junction $\left(i_{1}=i_{\mathrm{h}}=0\right)$

$$
v_{1}=\bar{v}+i_{\mathrm{f}}, \quad v_{\mathrm{h}}=-\sin \left(\bar{v} t+\int^{t} \mathrm{~d} t^{\prime} \cdot i_{\mathrm{f}}^{\prime}\right)
$$

from where one obtain directly expressions for the spectral density $s_{v}(\Omega)$ which are (2.24) and (2.29).

We obtain for case of the external hf signal $\left(i_{1}=0\right.$, $\left.i_{\mathrm{h}}=i^{(1)} \sin \Omega t\right)$

$$
i_{\mathrm{s}}=-i^{(1)} \sin \chi, \quad \chi=\delta \bar{i} . t+\chi^{(0)}-\pi / 2
$$

where $\delta \bar{i}=v-\Omega$ is a deviation of the current from autonomous value for $\bar{v}=\Omega$. We obtain from (5.18) and (5.20)

$$
\dot{\chi}+\frac{i^{(1)}}{2 \Omega} \sin \chi=\delta \bar{i}+i_{\mathrm{f}} .
$$

Since this equation is completely analogous to the basic eq. (2.12) is shows that the shape of the $i-v$ curve in the vicinity of the first Josephson step of the current at $\Omega \widetilde{>} 1$ is analogous to the shape of the $i-v$ curve $(2.10)$ in autonomous case [35], [76], [94], [95]. In this case fluctuations also smooth over the current step and during the analysis the expressions $(2.10)$ are completely valid if to substitute the intensity of fluctuations $\Gamma$ for one normalized with respect to the step value

$$
\begin{gathered}
\Gamma \rightarrow \Gamma^{(1)}=\Gamma /\left(i^{(1)} / 2 \Omega\right), \\
\langle\bar{v}\rangle \rightarrow\langle\overline{\dot{\chi}}>=\langle\bar{v}>-\Omega, \bar{i} \rightarrow \delta \bar{i} .
\end{gathered}
$$

5.4 THE « NON-JOSEPHSON » OSCILLATIONS AT OWN FREQUENCY OF THE CAVITY. - Let the external circuit at the frequency $\Omega$ has the high quality resonance $\left(Q_{\mathrm{h}}=\Omega / 2 h_{\mathrm{h}} \gg 1\right)$ of series type and the circuit resistance $r_{\mathrm{h}}$ corresponds to the resonant frequency. As we have seen in the part 3 if the bias voltage is somewhat less than $\Omega$ then the junction impedance for oscillations at frequency $\Omega$ has negative real part $\operatorname{Re} z<0$. If

$$
\operatorname{Re} z<-r_{\mathrm{h}}
$$

then the oscillations will arise at own frequency $\Omega$ of the circuit (really cavity) and this frequency is higher than frequency $\bar{v}$ of the Josephson oscillations taking place simultaneously in the junction. And if the condition of narrow bandwidth (3.31) is fulfilled then the Josephson oscillations practically do not excite the cavity. We will calculate a power of these " non-Josephson » oscillations and a variation of

(20) For the case $i_{1}=0, i_{\mathrm{f}}=0, i_{\mathrm{h}}=i^{(1)} \sin \Omega t$ such equations were used in the work [76]. 
the $i-v$ curve of the junction under action of these oscillations.

Let the current through the cavity again has the form $i_{\mathrm{e}}=i^{(1)} \sin \Omega t$. Then the eq. (5.21) is valid. On the other hand $v_{\mathrm{h}}=-r_{\mathrm{h}} i^{(1)} \sin \Omega t$ at resonant frequency and we have from (5.17)

$$
\left(1+r_{\mathrm{h}}\right) i^{(1)}+\sin \chi=0 .
$$

Substituting $\sin \chi$ from (5.21) to (5.24) and carrying out averaging procedure we have

$$
<\dot{\chi}>=\frac{1+r_{\mathrm{h}}}{2 \Omega}\left(i^{(1)}\right)^{2}
$$

This equation together with the expression (2.10) into which the substitutions (5.22) are introduced form the set of two algebraic equations for quantities $\langle\overline{\dot{\chi}}\rangle=\langle\bar{v}\rangle-\Omega$ and $i^{(1)}$.

The variations of $i-v$ curve of the junction and the power of oscillations at the own frequency of the cavity are shown at figure 15 . The shape of these curves depends only on the dimensionless factor $Q^{*}=Q /\left(1+r_{\mathrm{h}}\right)$, where $Q$ is the regeneration factor introduced earlier (see the part $3, Q=1 / 8 \Omega \Gamma$ ).

At small noise $\left(Q^{*} \gg 1\right)$ the variation of the $i-v$

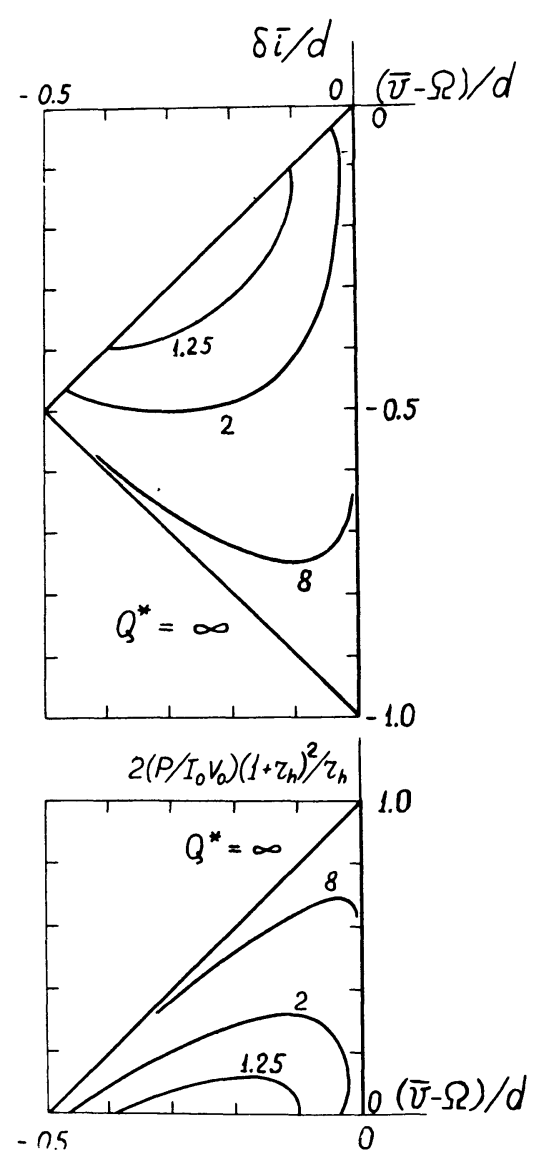

FIG. 15. - The variation of the $i-v$ curve of the junction at the excitation of the cavity at own ("non-Josephson ») frequency $\Omega \gg 1$. The parameter is the relative level of the fluctuations : $Q^{*}=1 / 8 \Omega \Gamma\left(1+r_{\mathrm{h}}\right)=d / 4 \Gamma$. The excitation takes place if $Q^{*}>1$. The power of the oscillations is also shown. curve has the shape of triangular well with the dimensions $d=1 / 2 \Omega\left(1+r_{\mathrm{h}}\right)$ in the direction of the current and $d / 2$ in the direction of the voltage, differential resistance at left slope of the well being equal to minus unity i. e. to $-R$ in dimensional units. The left slope of the well corresponds to the " non-Josephson " oscillations at the own frequency of the cavity and the right slope corresponds to the forced oscillations of the cavity under action of the Josephson oscillations of the junction [68]. As it follows from figure 15 the maximum power of the parametric (" non-Josephson ") radiation into the cavity at any matching (i. e. any $r_{\mathrm{h}}$ ) is exactly equal to the maximum power of the Josephson radiation $\left({ }^{21}\right)$.

The same result remains valid for $\Omega \approx 1$. The variation of the junction $i-v$ curve depending on $r_{\mathrm{h}}$ at $\Omega=0.3$ is shown at figure 16 . It is easy to obtain in case $r_{\mathrm{h}} \gg 1$ that $r_{\mathrm{d}}$ at left slope is

$$
r_{\mathrm{d}}=-\frac{\bar{i}}{\bar{v}}=-r_{\mathrm{d}}^{(0)}
$$

i. e. is exactly equal by modulus to the resistance of the autonomous junction at given bias voltage.

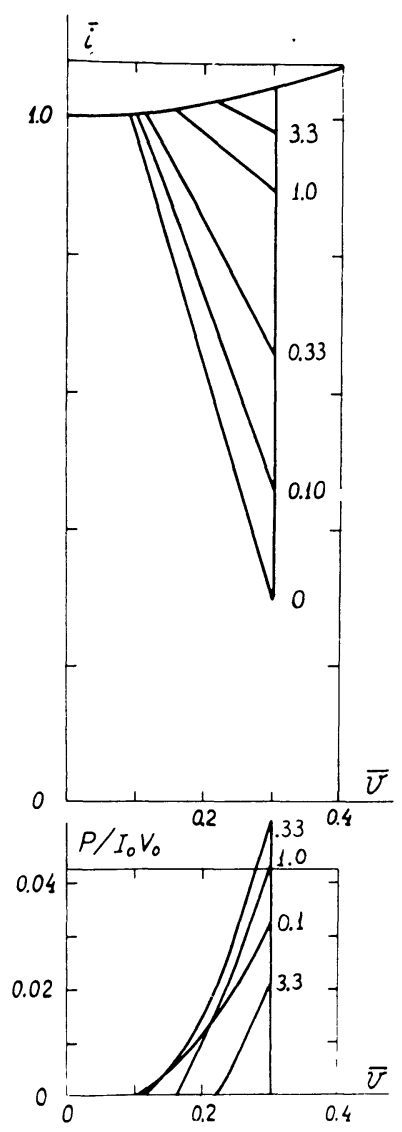

Fig. 16. - The same dependences as at figure 15 at $\Omega=0.3$ in the absence of fluctuations $\left(Q^{*} \gg 1\right)$. The values of the junction resistance $r_{\mathrm{h}}=R_{\mathrm{h}} / R$ are shown with the figures.

(21) The parametric oscillations will not take place only in wide-band external circuit when $h_{\mathrm{h}} \widetilde{>} d=1 / 2 \Omega\left(1+r_{\mathrm{h}}\right)$. 
The oscillations at the own frequency of the cavity for the S-c-S junctions at $\bar{v} \neq \Omega$ were observed recently by Ulrich and Kluth [96], [97] $\left({ }^{22}\right)$. The triangular wells at the $i-v$ characteristics were observed clearly in the works [68], [99], the expression (5.26) being satisfied well. Moreover the wells at the $i$ - $v$ curve corresponding to the self-detection during another experiments with the S-c-S junctions inserted into the cavity [41], [82] had the bandwidth much larger than the cavity bandwidth and were similar to ones shown at figures 15,16 . Therefore one has the reason to guess that in these experiments the " non-Josephson " oscillations also have taken place.

Note that the eq. (5.21) and (5.25) allow to obtain another interesting results. Even if the parameter $Q^{*}<1$ and therefore the parametric oscillations do not take place the anomalous impedance of the junction results in the variation of the forced Josephson oscillations at the cavity. For instance if we observe the processes in the cavity using narrow-band receiver (with bandwidth $\ll h_{\mathrm{h}}$ ) tuned at the own frequency of the cavity in the absence of the junction then the maximum reading from the receiver at the variation of the bias voltage will be observed not at $\bar{v}=\Omega$ but at $\bar{v}=\Omega-d / 2<\Omega$.

5.5 THE DOWN-CONVERSION WITH EXTERNAL PUMPING. - The properties of the S-c-S junctions at the action of strong external oscillations are using in the parametric down-converters (mixers) with external pumping [58], [66], [99]-[102] $\left({ }^{23}\right)$ where the variation of the parameter is producing not owing to the intrinsic Josephson oscillations but owing to intensive external pumping signal at the frequency $\Omega$ (with the amplitude $i_{\mathrm{e}}$ ). In addition to the pumping signal the input signal at frequency $\Omega+\Delta$ (usually $|\Delta| \ll 1, \Omega$ ) is applying to the junction and the lf signal at frequency $|\Delta|$ is picking up from the junction. Two regimes of operation in this case are possible. First is one when the operating point is located at some current step or superconducting region and second is one when the operating point is located at the portion of the $i-v$ curve between steps.

One would think that the location of the operating point at one of steps when the Josephson oscillations are synchronized by external source allows to avoid one of main shortcomings of converters with selfpumping, namely the finite bandwidth of the pumping and therefore of the output signal. However as it was shown in our analysis which will be published soon this regime has an essential shortcoming. To make narrower the bandwidth of oscillations of the

(22) Such oscillations in the Josephson structures were observed first in the tunnel junctions [98]. See also [54].

(23) In these works and also in our experiments with the conversion of $4 \mathrm{~mm}$ signal to $\mathrm{X}$-band range the combination frequency oscillations were observed directly in contrast to the works [73], [85] where only the steps at the $i$ - $v$ curve corresponding to combination frequency were observed. See also [90]. junction we should have the current of sufficiently large amplitude. At large current the step at which the operating point is located becomes almost vertical $\left(r_{\mathrm{d}} \rightarrow 0\right)$. Owing to this reason the lf output impedance of the converter becomes extremely low $\left({ }^{24}\right)$ and the matching of the junction with the if circuit becomes an exceptionally complicated problem. Even if good matching would be achieved the parameters of the down-converter will be almost the same as in case with self-pumping. Therefore though the realization of such regime is possible in principle it has not practical interest. The same conclusion was made by Kanter [66].

In the second regime the frequency of Josephson oscillations is not connected with frequency $\Omega$ by a definite relation and the self-pumping process does not take part in the oscillations process.

Let us consider this regime for the case of wideband hf circuit (3.22). In this case the effective hf impedance is again equal to unity (i. e. to the normal resistance of the junction) and the if output impedance is equal to the dc differential resistance $r_{\mathrm{d}}$ in the operating point. Writing down the expression for coupling current

$$
i_{\mathrm{e}}=i_{\Omega} \sin \Omega t+\frac{e}{1+r_{\mathrm{h}}} \sin (\Omega+\Delta) t,
$$

note that at small input signal $\left(e \ll\left(1+r_{\mathrm{h}}\right) i \Omega\right)$ one can represent it in a form of the signal at frequency $\Omega$ which amplitude is quantity slowly varying with time $\left({ }^{25}\right)$

$$
i^{(1)}=\sin \Omega t\left(i_{\Omega}+\frac{e}{1+r_{\mathrm{h}}} \cos \Delta t\right) .
$$

Let the operating point is located at the interval $\delta<\vec{v}>$ from the edge of the nearest step and

$$
|\delta<\bar{v}\rangle|\gg| \Delta \mid \text {. }
$$

Then reaching the steady-state value of the voltage $\langle\bar{v}\rangle$ across the junction will proceed in time $\sim|\delta\langle\bar{v}\rangle|^{-1}$ which is much less than the period of the variation of the effective amplitude of hf current $(2 \pi / \Delta)$. Therefore one can suppose that the variation of the $i-v$ curve at fixed current is

$$
\tilde{\bar{v}}=-\frac{e r_{\mathrm{d}}}{1+r_{\mathrm{h}}} \cos \Delta t, \quad \mu=\left(\frac{\partial<\bar{i}\rangle}{\partial i^{(1)}}\right)_{e=0} .
$$

Hence we have for the conversion factor in the absence of intrinsic losses in If and hf circuits $\left({ }^{26}\right)$

$$
\begin{gathered}
G=\alpha_{1} \alpha_{\mathrm{h}} \frac{\mu^{2} r_{\mathrm{d}}}{4}, \\
\alpha_{1}=\frac{4 r_{1} r_{\mathrm{d}}}{\left(r_{1}+r_{\mathrm{d}}\right)^{2}} \leqslant 1, \quad \alpha_{\mathrm{h}}=\frac{4 r_{\mathrm{h}}}{\left(1+r_{\mathrm{h}}\right)^{2}} \leqslant 1 .
\end{gathered}
$$

(24) It is that low impedance owing to which they have not observed experimentally a conversion at such regime.

(25) Such representation of the conversion mechanism was given first in the work [100].

(26) Similar expression was obtained in the works [66], [104]. 
Thus the matching has being reached of course at $r_{1}=r_{\mathrm{d}}, r_{\mathrm{h}}=1$. Using the expression (5.1) and also the results of calculation of the $i-v$ characteristic at $\Omega \approx 1$ (see for instance [89]) we obtain that at the correct choice of the operating point (sufficiently near to the edge of the $n$th step)

$$
G_{\max }= \begin{cases}\frac{r_{\mathrm{d}}}{16 \Omega^{2}}\left[2 J_{n}^{\prime}\left(\frac{i_{\Omega}}{\Omega}\right)\right]^{2}, & \Omega \approx 1, \\ \frac{r_{\mathrm{d}}}{4}, & \Omega \approx 1 .\end{cases}
$$

Since $\left(\left[2 J_{n}^{\prime}\left(i_{\Omega} / \Omega\right)\right]^{2}\right)_{\max } \simeq 1$ one can see from (6.32) and (3.27) that $G_{\max }$ for wide-band down-converter with external pumping at $\Omega \widetilde{>} 1$ is $r_{\mathrm{d}}$ times larger than for the similar converter with the self-pumping. For $\Omega \approx 1$ even the conversion with gain (at $r_{\mathrm{d}}>4$ ) is quite possible.

The maximum value of $r_{\mathrm{d}}$ depends on the noise level (see Fig. 1) and the values $r_{\mathrm{d}} \approx 10$ most probably are real (essentially at $\Omega \sim 1$ ).

Before finding noise parameters of the down-converter note that at $\Omega \widetilde{ } 1$ as it is seen from (5.21) the variation of the "difference phase " $\chi$ proceeds with the same manner as the variation of the phase in case of autonomous junction. Therefore carrying out the same calculations as in the part II we obtain that the expression (2.30) is valid if $r_{\mathrm{d}} \gg 1$. Hence the noise temperature of the down-converter reduced to the output is

$T_{\text {out }}=a r_{\mathrm{d}} T, \quad a=1+\frac{r_{\mathrm{h}}}{2\left(1+r_{\mathrm{h}}\right)^{2}} \simeq \frac{9}{8}$.

Corresponding calculations for case $\Omega \approx 1$ are not carried out yet but preliminary estimations show that the expression (5.33) is valid may be at somewhat other value of factor $a$. Therefore we have for the noise temperature reduced to the input

$$
\left(T_{\text {in }}\right)_{\min }= \begin{cases}9 \Omega^{2} T, & \Omega \widetilde{\sim} 1, \\ 2 a T, \quad a \simeq 1, & \Omega \approx 1 .\end{cases}
$$

The comparison of $(5.34)$ with the results for the down-converter with self-pumping (3.30) shows that the noise properties of both down-converters are almost the same.

The noise of the pump oscillator at the hf signal frequency is often appreciable source of noise in down-converter. Therefore the question about maximum possible frequency of if circuit $|\Delta|$ is important. According carried out above calculation $G$ does not depend on $\Delta$ until $|\Delta| \ll|\delta<\bar{v}>|$. Therefore to increase $|\Delta|_{\max }$ it is necessary to make larger the interval $|\delta\langle\bar{v}\rangle|$ from the operating point to the edge of the step. At

$\Omega \sim 1\left|r_{\mathrm{d}}\right|_{\max } \simeq$

$$
\simeq J_{n}\left(\frac{i \Omega}{\Omega}\right) \cdot|\delta<\bar{v}>|^{-1} \approx|\delta<\bar{v}>|^{-1} .
$$

Therefore

$$
|\Delta|_{\max } \simeq r_{\mathrm{d}}^{-1}, \quad(G|\Delta|)_{\max } \simeq\left(16 \Omega^{2}\right)^{-1} \text {. }
$$

At $\Omega \approx 1$ the variation of $r_{\mathrm{d}}$ is small at the whole of the portion of the $i-v$ curve between the steps and

$$
|\Delta|_{\max } \simeq \Omega / 2 \text {. }
$$

Thus $|\boldsymbol{\Delta}|_{\max }$ practically in all cases is sufficiently large (of order of the characteristic frequency of the junction or of the input signal frequency).

The numerical results for the down-converters with external pumping were obtained only in the works [58], [66], [103]. According to the communication of Kanter [66] the conversion loss about $10 \mathrm{~dB}$ was obtained at the conversion from $13 \mathrm{GHz}$ to $60 \mathrm{MHz}$ at $T=4.2 \mathrm{~K}$ in the work [103]. For any real junctions at $T=4.2 \mathrm{~K}\left(\omega_{0} / 2 \pi\right) \gg 13 \mathrm{GHz}$ i. e. $\Omega \ll 1$ and the theory predicts in this case the value $\left(G^{-1}\right)_{\min } \simeq 0 \mathrm{~dB}$. So the mentioned experimental loss should be put down to the matching. The similar conclusion should be done for the results of the works [58], [66]. The minimum loss $11 \mathrm{~dB}$ for the conversion from $34 \mathrm{GHz}$ to $300 \mathrm{MHz}$ was obtained in the work [58] and the losses $10 \mathrm{~dB}$ and $14 \mathrm{~dB}$ for the conversion from $90 \mathrm{GHz}$ to $15 \mathrm{GHz}$ and $30 \mathrm{MHz}$ respectively were obtained in the work [66]. Note that in the last case the parameter $\Omega$ was comparable with unity.

The value of the NEP $\sim 10^{-18} \mathrm{~W} / \mathrm{Hz}^{1 / 2}$ is given also in the work [103]. It corresponds to $T_{\text {in }} \simeq 6 \times 10^{4} \mathrm{~K}$ while taking into account the conversion loss one can obtain from (5.34) at $a=2$ the noise temperature $T_{\text {in }} \simeq 3 \times 10^{2} \mathrm{~K}$. It is difficult to explain now such large discrepancy.

6. The discussion of the results. The conclusion. 6.1 THE NOISE PROPERTIES OF THE RECEIVING DEVICES USING THE S-c-S JUNCTIONS. - We have obtained in the parts 3, 5 the limit characteristics including the noise characteristics of different receiving devices : detectors, amplifiers, down-converters with self- and external pumping. The operation regimes of these devices are different to a considerable extent however all of them have identical to a certain degree noise characteristics.

Indeed let any of these devices is the first stage of the receiver, and following stages of it are ideal i. e. do not insert additional noise. Then the noise equivalent power for any of these receivers as it follows from (3.30), (3.38), (3.39), (4.20), (4.26), (4.31), (4.32) can be written down in the form

$$
\begin{gathered}
(N E P)_{\min }=F k T\left(H_{\mathrm{h}} H_{\mathrm{M}}\right)^{1 / 2}, \\
F= \begin{cases}A, & \Omega \approx 1 \\
B \Omega^{2}, & \Omega \approx 1\end{cases}
\end{gathered}
$$

where $A$ and $B$ are factors, slightly different for different regimes but each of order of unity, $H_{\mathrm{h}}$ 
is effective hf bandwidth of given device, $H_{\mathrm{M}}$ is output bandwidth (reciprocal averaging time).

The expression (6.1) has the same structure as well-known formula for ideal receiver at frequency

$$
\begin{gathered}
\omega \approx k T / \hbar \\
(N E P)_{\min }=C k T 2 \pi\left(H_{\mathrm{h}} H_{\mathrm{M}}\right)^{1 / 2},
\end{gathered}
$$

where the factor $C$ (of order of unity) depends on the method of signal processing. This formula is valid when the receiver is under action of the hf noise corresponding to Nyquist formula at the temperature $T$ and the bandwidth $H_{\mathrm{h}}$, and it is not valid when the If noise at output of the receiver prevails over the hf noise.

The most essential peculiarity of the expression (6.1) consists in the increasing of $(N E P)_{\min }$, or the noise temperature which is equivalent to the $N E P$, of the Josephson receiving devices proportionally to $\Omega^{2}$ at $\Omega \sim 1$. This increasing is connected with different reasons for different devices.

The increasing of the $(N E P)_{\min }$ for the detectors in wide-band and self-selection regimes and also for the down-converters with wide-band hf circuit (3.22) is connected with the decreasing of down-conversion efficiency ( $G$ for down-converters and $\eta$ for detectors) at $\Omega>1$. In next turn it is caused by the most part of the signal energy at $\Omega \gg 1$ being dissipated in the normal resistance of the junction.

The responsivity of detectors and the efficiency of amplifiers and down-converters at regenerative regime of operation become so high that the influence of the If noise is small. One would think that in this case the conditions are fulfilled at which the formula (6.2) is valid. However as it is seen for instance from (2.43) the penetration of the wings of the Josephson oscillations line into the hf bandwidth of the device results in the effective hf noise temperature being equal to

$$
T_{\mathrm{e}}=T\left(1+1 / 4 \Delta^{2}\right)
$$

even at $\Gamma \rightarrow 0$. Since to achieve the regeneration at $\Omega \gg 1$ as it follows from (3.11) the satisfying of the inequality $\Delta<1 / 4 \Omega$ is necessary then

$$
\left(T_{\mathrm{e}}\right)_{\min } \simeq 16 \Omega^{2} T
$$

and this is that which results in increasing of the NEP.

Thus achieving the limit value of the NEP (6.2) (i. e. of the noise temperature equal to the physical temperature $T$ ) for considered receiving devices is possible only at frequencies which are less than the characteristic frequency $\omega_{0}$ of the junction. Therefore the main requirement to the junctions for microwave application is high value of $\omega_{0}$, i. e. $V_{0}$.

The parameter $\omega_{0}$ is of importance also for the other applications of S-c-S junctions. It can be shown for instance that the noise equivalent magnetic flux in Josephson magnetometers (SQUID's) is equal to $\varphi_{\min } \simeq \varphi_{0}\left(H_{\mathrm{M}} / \omega_{0}\right)^{1 / 2}, \varphi_{0}$ being the flux quantum and $H_{\mathrm{M}}$, the bandwidth of measurement circuit.

6.2 THE RESISTANCE OF THE JUNCTION AND THE PROBLEM OF THE SHUNTING. - The requirements for the normal resistance $R$ which is second important parameter of the junction are contradictory in all cases except the case of the wide-band detector. Indeed the best characteristics of it are achievable at the resistance $R \simeq R_{\mathrm{f}}$ values of which at $T=4.2 \mathrm{~K}$ are of order of $10^{2}-10^{3} \mathrm{ohms}$. The hf matching of such junction is not problem since the wave impedance of usual microwave circuits is of order of wave impedance of the free space $\left(R_{0}=120 \pi \mathrm{ohm}\right)\left({ }^{27}\right)$. Of course the technology of reliable and reproducible junctions with such resistance and also shielding of the junctions with respect to external interferencies are problems.

For the rest of operation regimes of the S-c-S junctions it is necessary, on the one hand, to have $R$ of order of at least $10^{1}-10^{2} \mathrm{ohms}$ for convenience of $\mathrm{hf}$ and If matching and, on the other hand, to decrease the value of $\Gamma$ for decreasing the oscillations bandwidth. So for instance if one needs the selfselective detector with relative bandwidth $\sim 10^{-4}$ then even in the absence of if interferences the corresponding value of $R$ should be $\sim 10^{-1} \mathrm{ohm}$.

Fortunately thermal fluctuations (1.8) actually are not unavoidable. Like the if interferences they can be reduced strongly owing to shunting the junction with small external resistance $R_{\mathrm{s}} \ll R$ even being at the same temperature [34], [42], [106], [107]. Besides the value of shunt resistance the upper frequency $\omega_{\mathrm{s}}$ untill which the shunt impedance is of order of $R_{\mathrm{d}}$ is also important parameter of the shunt. So in the simplest case $\omega_{\mathrm{s}} \simeq R_{\mathrm{d}} / L_{\mathrm{s}}$, where $L_{\mathrm{s}}$ is the inductance of the conductor forming the shunt.

As we have seen in the part II the line bandwidth $\Gamma_{1}$ of the Josephson oscillations at $\Omega \gg 1$ depends on fluctuations spectral components only with frequencies less than $\Gamma_{1}$. For all devices except wide-band detector required values of $\Gamma_{1} \ll \Omega,|\Delta|$, i. e. the line bandwidth of the oscillations should be much less than frequencies of hf and if processes. Therefore is to choose the shunt so that

$$
\Gamma_{1} \ll \omega_{\mathrm{s}} / \omega_{0} \ll \Omega,|\Delta|,
$$

then reducing $\Gamma_{1}$ in $\left(R_{\mathrm{s}} / R\right)$ times is taking place and another properties of the junction do not change.

(27) It is interesting to note that, as $e V_{0} \simeq k T$ always for superconductors, the proximity of values $R_{\mathrm{f}} / 2 \simeq \hbar / 4 e^{2}$ and $R_{0}=4 \pi / c$ one to another is the consequence of the fact that the constant of electromagnetic interaction $\alpha=e^{2} / \hbar c \simeq 1 / 137$ is only about two orders less than unity [105]. 
The main difficulty for selective regimes of detectors consists in the fact that the shunt should not act at the frequency of which the detected signal has being picked up. There are two ways to avoid this difficulty. The first one consists in using radio frequency (rf) biasing (cross modulation regime) i. e. when the information about the variation of the $i-v$ curve is picking up not in the form of variation of the bias voltage but in the form of variation of the junction impedance for the rf signal with the voltage amplitude across the junction $\sim \Gamma_{1} V_{0}$. Such method was used in the works [72]-[74].

The second way is also available in principle when a series resonance circuit tuned at frequency of the detected signal is inserted in series with the shunt.

6.3 THE PARAMETERS OF THE DEVICES USING THE SHUNTING. - Let the conditions (6.5) are fulfilled. Define

$$
\Gamma_{\mathrm{s}}=\Gamma\left(R_{\mathrm{s}} / R\right)
$$

It is easy to see that in this case the expression (2.28) becomes

$$
\Gamma_{1 \mathrm{~s}}=\Gamma_{\mathrm{s}} \times\left(T_{\mathrm{s}} / T\right)
$$

and the basic expressions (2.43), (3.14) remain in former form at the substitution $\Gamma_{1} \rightarrow \Gamma_{1 \mathrm{~s}}$. Using them we obtain the following results if $T_{\mathrm{s}}=T$.

6.3.1 The non-regenerative (wide-band) down-converter with self-pumping. - The expressions (3.24)(3.30) remain valid.

6.3.2 The regenerative amplifier. - The expressions (3.32), (3.33), (3.36) for the efficiency remain valid at taking into account the transformation of $Q$

$$
Q \rightarrow 1 / 4(\bar{i}+\bar{v}) r_{\mathrm{d}} \Gamma
$$

and for the noise temperature we obtain from (3.34)

$$
\begin{aligned}
& \frac{T_{\mathrm{in}}}{T}=16 \Omega^{2}\left(1-Q_{1}^{-2}\right)^{-1} T . \\
& Q_{1}=\frac{\omega_{0}}{4(\bar{i}+\bar{v}) \omega_{\mathrm{s}}}
\end{aligned}
$$

6.3.3 The regenerative down-converter with selfpumping. - The same results take place as in previous case.

6.3.4 The detector in the self-selection regime. The expressions (4.23), (4.25) are valid if to produce the substitution $I_{\mathrm{fh}} \rightarrow I_{\mathrm{fh}}\left(\Gamma_{\mathrm{s}} / \Gamma\right)$. Instead of (4.26) we obtain at $|\Delta|=\Gamma_{1 \mathrm{~s}}, r_{\mathrm{h}}=1$ :

$$
(N E P)_{\min }=(8 / \sqrt{\pi}) k T\left(H_{\mathrm{h}} H_{\mathrm{M}}\right)^{1 / 2} \Omega^{2} \text {. }
$$

\subsubsection{The detector in regenerative regime (external}

selection). - Instead of $Q$ in the expression (4.32) we obtain

$$
Q \rightarrow Q_{1}
$$

at the same conditions as for the regenerative amplifier.

6.3.6 The down-converter with external pumping. All expressions for $G$ remain valid but is is necessary to take into account that the values of $r_{\mathrm{d}}$ increase at decreasing of fluctuations.

The advantages of the shunting are so significant that it is difficult to imagine a case when it would not be advantageous.

6.4 THE COMPARISON OF THE RECEIVING DEVICES USING THE S-c-S JUNCTIONS. - It is natural for the conclusion to compare the obtained characteristics of different receiving devices using single S-c-S junction and to attempt to choose the most suitable one among them. Of course this choice depends on a given problem and in first turn on the required hf bandwidth of the receiver.

In case of devices like radiometers where maximum hf bandwidth is desirable the Josephson detector operating in wide-band regime is preferable because its own bandwidth at $\Omega \simeq 1$ and optimal values of $\bar{v}$ and $\Gamma$ is of order of $\omega_{0}$. So taking for example again two-millimeter waveband range and values $T=4.2 \mathrm{~K}$, $V_{0}=300 \mu \mathrm{V}, \alpha_{\mathrm{n}}=1$ we have from figure 12

$$
(N E P)_{\min } \simeq 6 \times 10^{-16} \mathrm{~W} / \mathrm{Hz}^{1 / 2}
$$

at the bandwidth $\sim 50 \mathrm{GHz}$. We see that the temperature sensitivity of the wideband detector using as a radiometer can reach value of order of $10^{-3} \mathrm{~K}$ that is essentially better than for operating now competing devices.

In case of radiometers which hf bandwidth is limited by the given problem or by the bandwidth of the intermediate frequency (if) amplifier, really of order not more than several $\mathrm{GHz}$ for centimeter waveband range, the application of the downconverters with external pumping is possible. Very low conversion losses can be achieved using such device (even gain is available at $r_{\mathrm{d}}>4$ ) so that the requirements to the if amplifiers for them are not so strict. So for instance at mentioned above parameters and if bandwidth $1 \mathrm{GHz}$ we have at optimal shunting for the $(N E P)_{\min } \simeq 10^{-17} \mathrm{~W} / \mathrm{Hz}^{1 / 2}$ that corresponds to the temperature sensitivity $\delta T_{\mathrm{f}} \sim 10^{-3} \mathrm{~K}$ taking into account image channel. To realize such figure it is necessary to have the if amplifier with effective noise temperature $\approx 20 \mathrm{~K}$. One should keep in mind however that properties of such down-converters will deteriorate at frequencies higher than $\omega_{0}$ even using the shunting.

For the purpose of the measurements of spectra (especially line spectra) of signals the wide-band down-converters with self-pumping and the selfselective detectors are promising. The latter is appa- 
rently preferable because it is simpler (the if amplifier is absent) and as it follows from (6.8), (6.11) can ensure the same parameters as the down-converter. For instance for real $\mathrm{hf}$ bandwidth in the shunting regime equal to $\Gamma_{1 \mathrm{~s}} / \Omega=10^{-3}$ we have for the detector

$(N E P)_{\min } \simeq 2 \times 10^{-17} \mathrm{~W} / \mathrm{Hz}^{1 / 2}, \delta T_{\mathrm{f}} \sim 5 \times 10^{-3} \mathrm{~K}$.

The most reasonable field of application of the regenerative devices with negative resistance (amplifiers, down-converters and detectors) is the receiving of signals in the fixed narrow band because the retuning of the external cavity, which is necessary in this case, keeping constant and high level of the regeneration is special problem. The important advantage of the devices with regeneration is their capability to operate with the shunt at $\Omega \sim 1$ maintaining comparatively high conversion efficiency. If just the measurement of the signal power is important the detector has advantage again in comparison with others. For instance at the bandwidth of the cavity without the junction $3 \mathrm{GHz}\left(Q \sim 10^{2}\right)$ and the degree of the regeneration $20 \mathrm{~dB}$ we have $(N E P)_{\min } \simeq 3 \times 10^{-18} \mathrm{~W} / \mathrm{Hz}^{1 / 2}$ in the bandwidth $30 \mathrm{MHz}$.

The down-converters with self-pumping (nonregenerative and regenerative) are preferable instead of detectors (self-selective and regenerative respectively) only in case when the information about the signal phase is necessary or instead of the downconverters with external pumping in two cases, one when receiving system is compact and other when operating frequencies are high $(\Omega \gg 1)$.

Thus practically each of the studied here regimes of operation has its own field of application. Using them one can have the receiving devices with high performance in one's special case. Note in connection with this that the proximity of the effective temperature of these devices and $T$ means that the registration of signals with frequencies $\omega \simeq k T / \hbar$ at the single photon level is possible [105]. However as it was shown the practical realization of this possibility is quite different from one supposed in the work [105]. We think that using superconducting cavities of high quality in non-steady-state regime [108] with the Josephson receiving devices it will be possible to detect single photon signals even at frequencies $\omega \ll k T / \hbar$.

We have described here only the systems each inserting one contact. It is well known that the systems of some (many) Josephson junctions can have very high parameters, essentially as the elements of wide-band detectors. Having no room for detailed discussion of these questions we put here only one of obtained results. The limit parameters of wideband detector with many S-c-S junctions (with granular superconductor for instance) cannot be better than ones for one-junction detector. However, the utilization of many-junction detectors can be more advantageous in the case of strong external interferences and also when many-mode radiation of submillimeter range is received.

Acknowledgments. - The authors wish to mark gratefully the participation of Margolin, N. M., Shtykov, V. D. and also Leschenko, G. F., Yacobson, L. A., Avakyan, R. S., D'yakov, V. P., Kupriyanov, M. Yu. and Spitzin, A. M. in the investigations.

\section{References}

[1] Kulik, I. O., Yanson, I. K., The Josephson effect in the superconducting tunnel structures (« Nauka » Publishing House, Moscow) 1970 (in Russian).

[2] Zimmerman, J. E., Proc. 1972 Appl. S.-C. Conf., IEEE Conf. Rec. NCHO 682-5-TABSC, (1972) 544.

[3] Gregers-Hansen, P. E., Levinsen, M. T., Pedersen, L., Suostrom, C. J., Solid. State Commun. 9 (1971) 661.

[4] Gregers-Hansen, P. E., Levinsen, M. T., Phys. Rev. Lett. 27 (1971) 847.

[5] Song, Y., Rochlin, G. L., Phys. Rev. Lett. 29 (1972) 416.

[6] Gregers-Hansen, P. E., Levinsen, M. T., Pedersen, G. F., J. Low Temp. Phys. 7 (1972) 99.

[7] Gregers-Hansen, P. E., Hendricks, E., Levinsen, M. T., Pedersen, G. F., Proc. 1972 Appl. S.-C. Conf., IEEE Conf. Rec. NCHO 682-5-TABSC (1972) 597.

[8] Laibowitz, R. B., Hatzakis, M., Bull. Am. Phys. Soc. 18 (1973) 464.

[9] Blackwell, L. A., Kotzebue, K. L., SemiconductorDiode Parametric Amplifiers (Prentice-Hall, Inc., Englewood Cliffs, N. J.) 1961.

[10] Etrin, V. S., Gershenzon, E. M., Semiconductor-Diode Parametric Systems («Sovjetskoje Radio» Publishing House, Moscow) 1964 (in Russian).
[11] Penfield, P., Rafuse, R. P., Varactor Applications, MIT Press, 1962.

[12] Torrey, H. C., Whitmer, C. A., Crystal Rectifiers, MIT Rad. Lab. Ser. (McGraw-Hill Book Co, Inc., New York) 15 (1948).

[13] Kanter, H., Vernon, F. L., Appl. Phys. Lett. 16 (1970) 115 ; Phys. Rev. Lett. 25 (1970) 588.

[14] Katz, R. M., Rose, K., Proc. IEEE 61 (1973) 55.

[15] Feynmann, R. P., Lectures on Physics 3 (1963) 21, Addison-Wesley.

[16] Aslamazov, L. G., Larkin, A. I., Zh. Eksp.\& Teor. Fiz., Pis'ma Red. 9 (1969) 150 (in Russian).

[17] Gor'kov, L. P., Eliashberg, G. M., Zh. Eksp. \& Teor. Fiz., Pis'ma Red. 8 (1968) 329 (in Russian).

[18] Ambegaokar, V., Baratoff, A., Phys. Rev. Lett. 10 (1963) 486; 11 (1963) 104.

[19] Kirchman, R. K., Notarys, H. A., Mercereau, J. E., Phys. Lett. 34A (1971) 209.

[20] Kirchman, R. K., Mercereau, J. E., Phys. Lett. 35A (1971) 177.

[21] Notarys, H. A., Mercereau, J. E., Physica 55 (1971) 424.

[22] Kirchman, R. K., Proc. 1972 Appl. S.-C. Conf., IEEE Conf. Rec. NCHO 682-5-TABSC (1972) 707. 
[23] Clarke, J., Proc. R. Soc. A 308 (1969) 447.

[24] Bondarenko, S. I., Dmitrenko, I. M., Balanov, E. I., Fiz. Tver. Tel. 12 (1970) 1417 (in Russian).

[25] IsHII, C., Progress Theor. Phys. 44 (1970) 1525.

[26] Greensppon, S., Smith, H. J. T., Can. J. Phys. 49 (1971) 1350.

[27] Clarke, J., Phys. Rev. B 4 (1971) 2963.

[28] Bardeen, J., Johnson, J. L., Phys. Rev. B 5 (1972) 72.

[29] Taguchi, I., Yoshioka, H., J. Phys. Soc. Japan 33 (1972) $92 ; 34$ (1973) 1106.

[30] Aslamazov, L. G., Larkin, A. I., Ovchinnikov, Yu. N., Zh. Eksp. \& Teor. Fiz. 55 (1968) 323 (in Russian).

[31] Stewart, W. S., Appl. Phys. Lett. 12 (1968) 277.

[32] MCCumber, D. E., J. Appl. Phys. 39 (1968) 3113.

[33] Levinsen, M. T., private communication.

[34] LaRkin, A. I., Ovchinnikov, Yu. N., Zh. Eksp.\& Teor. Fiz. 53 (1967) 2159 (in Russian).

[35] Stephen, M. J., Phys. Rev. 182 (1969) 531 ; 186 (1969) 393.

[36] Likharev, K. K., Semenov, V. K., Zh. Eksp. \& Teor. Fiz., Pis'ma Red. 15 (1972) 625 (in Russian).

[37] Kanter, H., Vernon, F. L., Phys. Rev. B 2 (1970) 4694.

[38] Likharev, K. K., Semenov, V. K., Radiotekhnika $i$ Elektronika 18 (1973) 1757 (in Russian).

[39] Blaney, T. G., Radio El. Eng. 42 (1972) 303.

[40] Richards, P. L., Auracher, F., Van Duzer, T., Proc. IEEE 61 (1973) 36.

[41] Dayem, A. H., Grimes, C. C., Appl. Phys. Lett. 9 (1966) 47.

[42] Zimmerman, J. E., Cowen, J. A., Silver, A. H., Appl. Phys. Lett. 9 (1966) 353.

[43] Krasnopjlin, I. Ya., Khaikin, M. S., Zh. Eksp. \& Teor. Fiz., Pis'ma Red. 6 (1967) 633 (in Russian).

[44] Vystavkin, A. N., Gubankov, V. N., Kuzmin, L. S., Likharev, K. K., Migulin, V. V., Proc. 2-d Cornell Electrical Eng. Conf., 315, Ithaca, N. Y., 1971.

[45] Adde, R., Vernet, G., J. Appl. Phys. 43 (1972) 2405.

[46] Stratonovich, R. L., Topics in the Theory of Random Noise (Gordon and Breach, N. Y.) 1967.

[47] Ambegaokar, V., Halperin, B. I., Phys. Rev. Lett. 22 (1969) 1364.

[48] Gubankov, V. N., Likharev, K. K., Margolin, N. M., Fiz. Tver. Tel. 14 (1972) 953 (in Russian).

[49] Gubankov, V. N., Margolin, N. M., Filimonov, A. B., Fiz. Tver. Tel. 15 (1973) 1258 (in Russian).

[50] Malakhov, A. N., Fluctuations in Self-Oscillating Systems, (« Nauka » Publishing House, Moscow) (1968) (in Russian).

[51] Vernet, G., Adde, R., Appl. Phys. Lett. 19 (1971) 195.

[52] Vystavkin, A. N., Gubankov, V. N., Leschenko, G. F., LikHAReV, K. K., Migulin, V. V., Radiotekhnika $i$ Elektronika 15 (1970) 2404 (in Russian).

[53] Werthamer, N. R., Shapiro, S., Phys. Rev. 164 (1967) 523.

[54] Likharev, K. K., Vestnik Moskovskogo Univ. 6 (1968) 104 (in Russian).

[55] Russer, P., Arch. Electr. Übergrag. 23 (1969) 417.

[56] Russer, P., Proc. IEEE 59 (1971) 282.

[57] Vystavkin, A. N., Gubankov, V. N., Kuzmin, L. S., Likharev, K. K., Migulin, V. V., Radiotekhnika $i$ Elektronika 17 (1972) 896 (in Russian).

[58] Jenkins, V., Parker, E. A., Little, L. T., El. Letters 8 (1972) 540.

[59] Ulrich, B. T., Lee, T., Proc. 1972 Appl. S.-C. Conf., IEEE Conf. Rec. NCHO 682-5-TABSC (1972) 719.

[60] Landau, L. D., Lifshitz, E. M., Electrodynamics of Continuous Media, Gostekhizdat, Moscow, 1957 (in Russian).
[61] Kanter, H., Vernon, F. L., Phys. Lett. A 35 (1971) 349 ; J. Appl. Phys. 43 (1972) 3174.

[62] Auracher, F., Van Duzer, T., J. Appl. Phys. 44 (1973) 848.

[63] Kalashnik, L. I., Kislov, A. M., Kulik, I. O., Livshitz, E. M., Maskov, K. V., Motornaya, A. A., $Z \boldsymbol{h}$. Tekhn. Fiz. 42 (1972) 1296 (in Russian).

[64] Vystavkin, A. N., Gubankov, V. N., Kuzmin, L. S., LikhareV, K. K., Migulin, V. V., Spitzin, A. M., Zh. Eksp. \& Teor. Fiz., Pis'ma Red. 17 (1973) 284 (in Russian).

[65] Kanter, H., Appl. Phys. Lett. 23 (1973) 350.

[66] KaNTER, H., to be published.

[67] Zimmerman, J. E., J. Appl. Phys. 41 (1970) 1589.

[68] Longacre, A., Proc. 1972 Appl. S.-C. Conf., IEEE Conf. Rec. NCHO 682-5-TABSC (1972) 707.

[69] NAD', F. YA., Radiotekhnika i Elektronika 17 (1972) 2360 (in Russian).

[70] Grimes, C. C., Richards, P. L., Shapiro, S., Phys. Rev. Lett. 17 (1966) 431 ; J. Appl. Phys. 39 (1968) 3905.

[71] Richards, P. L., Sterling, S. A., Appl. Phys. Lett. 14 (1969) 394.

[72] Blaney, T. G., Phys. Lett. A 37 (1971) 19.

[73] Blaney, T. G., Bradley, C. C., J. Phys. D 5 (1972) 180.

[74] Fife, A. A., Gygax, S., J. Appl. Phys. 43 (1972) 2391.

[75] Divin, Yu. YA., NAD', F. YA., Radiotekhnika i Elektronika 18 (1973) 879 (in Russian).

[76] Volkov, A. F., NaD', F. YA., Zh. Eksp. \& Teor. Fiz., Pis'ma Red. 11 (1970) 92 (in Russian).

[77] Oнta, H., J. Appl. Phys. 43 (1972) 5162.

[78] Vasenko, S. A., Radiotekhnika i Elektronika 18 (1973) 1694 (in Russian).

[79] Dritrenko, I. M., Bevza, Yu. G., Mikhailov, V. A., Zh. Eksp. \& Teor. Fiz., Pis'ma Red. 17 (1973) 8 (in Russian).

[80] Likharev, K. K., Semenov, V. K., Radiotekhnika $i$ Elektronika 17 (1972) 1983 (in Russian).

[81] Volkov, A. F., Radioteknika i Elektronika 17 (1972) 2581 (in Russian).

[82] De Ouboter, R. B., Kraan, W. H., Waele, A. Th., OMAR, M. N., Physica 35 (1967) 335.

[83] Zimmerman, J. E., J. Appl. Phys. 42 (1971) 30.

[84] Bondarenko, S. I., Dmitrenko, I. M., Narbut, T. P., Fiz. Tver. Tel. 14 (1972) 354 (in Russian).

[85] McDonald, D. C., Kose, V. F., Evenson, K. M., Wells, J. C., Cupp, J. D., Appl. Phys. Lett. 15 (1969) 121.

[86] McDonald, D. C., Evenson, K. M., Wells, J. C., Cupp, J. D., J. Appl. Phys. 42 (1971) 179.

[87] Russer, P., Acta Phys. Austriaca 32 (1970) 373 ; J. Appl. Phys. 43 (1972) 2008.

[88] Fack, H., Kose, V., Schader, H. J., Messtechnik 79 (1971) 31 ; J. Appl. Phys. 42 (1971) 320.

[89] Likharev, K. K., Semenov, V. K., Radiotekhnika $i$ Elektronika 16 (1971) 2167 (in Russian).

[90] Russer, P. H., Bayegan, H., Proc. IEEE 61 (1973) 46.

[91] Hamilton, C. A., Johnson, E. G., Phys. Lett. A 41 (1972) 393.

[92] FaCK, H., Kose, V., J. Appl. Phys. 42 (1971) 322.

[93] Elsley, R. K., Sievers, A. J., Proc. 1972, Appl. S.-C.Conf., IEEE Conf. Rec. NCHO 682-5-TABSC (1972) 716.

[94] Kose, V. E., Sullivan, D. B., J. Appl. Phys. 41 (1970) 169.

[95] Henkels, N. H., Webi, W. W., Phys. Rev. Lett. 26 (1971) 1164.

[96] Ulrich, B. T., Phys. Lett. 42A (1972) 119.

[97] Ulrich, B. T., Kluth, E. O., Proc. IEEE 61 (1973) 51.

[98] Langenberg, D. N., Parker, W. H., Taylor, B. N., Phys. Lett. 22 (1966) 259. 
[99] LONGACRE, A., Electronics, 1 March 1971, p. 44.

[100] Grimes, C. C., Shapiro, S., Phys. Rev. 169 (1968) 397. [101] McDonald, D. C., Risley, A. S., Cupp, J. C., Evenson, K. M., Phys. Rev. Lett. 18 (1971) 162.

[102] Blaney, T. G., Bradley, C. C., EdWards, G. J., KNight, D. J. E., Phys. Lett. A 36 (1971) 285.

[103] DiNardo, A. L., Sard, E., J. Appl. Phys. 42 (1971) 105. [104] Auracher, F., Van Duzer, T., Proc. 1972 Appl. S.-C. Conf., IEEE Conf. Rec. NCHO 682-5-TABSC, (1972) 603.
[105] Chiao, R. Y., Phys. Lett. A 33 (1969) 177.

[106] Zimmerman, J. E., Silver, A. H., J. Appl. Phys. 39 (1968) 2679.

[107] Kanter, H., Silver, A. H., Appl. Phys. Lett. 19 (1971) 516 ; Proc. 1972 Appl. S.-C. Conf., IEEE Conf. Rec. NCHO 682-5-TABSC (1972) 592.

[108] Braginsky, V. B., Physical Experiments with Probe Bodies (« Nauka » Publishing House, Moscow) 1970 (in Russian). 